Transionospheric

Propagation Code (TIPC)

Robert Roussel-Dupré

Thomas A. Kelley 


\title{
TRANSIONOSPHERIC PROPAGATION CODE (TIPC)
}

by

\author{
Robert Roussel-Dupré and Thomas A. Kelley
}

\begin{abstract}
The Transionospheric Propagation Code is a computer program developed at Los Alamos National Lab to perform certain tasks related to the d tection of VHF signals following propagation through the ionosphere. The code is written in Fortran 77, runs interactively and was designed to be as machine independent as possible. A menu for. mat in which the user is prompted to supply appropriate parameters for a given task has been adopted for the input while the output is primarily in the form of graphics. The user has the option of selectirin from five basic tasks, namely (1) Transionospheric propagation, (2) Signal filtering, (3) Signal processing, (4) DTOA study, and (5) DTOA uncertainty study. For the first task a specified signal is convolved against the impulse response function or the ionosphere to obtain the transionospheric signal. The user is given a choice of four analytic forms for the input pulse or of supplying a tabular form. The option of adding Gaussian-distribuied white noise or spectral noise to the input signal is also provided. The deterministic ionosphere is characterized to first order in terms of a total electron content (TEC) along the propagation path. In addition, a scattering model parameteized in terms of a frequency coherence bandwidth is also available. In thn second task, detection is simulated by convolving a given filter response against the transionospheric signal. The user is given a choice of a wideband filter or a narrowband Gaussian filter. It is also possible to input a filter response. The third task provides for quadrature detection, envelope detection, and three different techniques for time-tagging the arrival of the transionospheric signal at specified receivers. The latter algorithms can be used to determine a TEC and thus take out the effects of the ionosphere to first order. Task four allows the user to construct a table of delta imes-of-arrival (DTOAs) vs. TECs for a specified pair of receivers. In this way, a TEC can be determined by interpolation for any given measur ement of DTOA. The fifth task can be used to study the effects of noise and/or scattering on the ability of a particular time-tagging algorithm to measure an accurate DTOA for a given set of receivers. A detailed description of the capabilities and inherent limitations of TIPC is presented. Future enhancements to improve the utility of the code and to address the issue of signal reconstruction are discussed.
\end{abstract}




\section{INTRODUCTION}

The purpose of this report is to outline the capabilities and limitations of a computer program (TIPC) developed in the Atmospheric Sciences group at Los Alanos National Lab to perform certain tasks related to the detection of VHF signals following propagation through the ionosphere. The input to this code is menu driven, allowing many options for individual tasks. Once the input is specified the task is executed and the relevant information is plotted both interactively and in a disk file (termed metafile in the Los Alamos computing enviromment) that is stored and available for post processing. Thus the relevant output in the present version of TIPC is primarily in the form of graphics. For each run a paraneter file containing the input data is created. This can be read in for future runs or printed out for a record of TIPC runs. TIPC can also be executed in batch mode by means of command files under the VAX VMS operating system. Although TIPC was originally designed to operate on a VAX, it can easily be converted to run on other systems and more generic routines have been incorporated to facilitate this process.

Many of the algorithms used in TIPC either were written specifically to address a particular aspect of RF propagation or are generic routines extracted from the Common Los Alamos Mathematical Software (CLAMS) library or available through standard math libraries (e.g., IMSL). In addition two computer codes written at other institutions, namely CIRF (Wittwer, Defense Nuclear Agency) and IRI (Rawer et al., 1978a and b; Bilitza, 1986: Rawer and Bradley, 1987) were incorporated into TIPC. A brief discussion of these codes along with appropriate references are included in this report. Mure recently CIRF has been replaced with a more efficient algorithm that reduces the computing time significantly. The graphics package initially used in TIPC, DISSPLA ${ }^{T M}$, has been replaced by more readily available software developed at the National Center for Atmospheric Research (NCAR graphics). All of the coding in TIPC is standard Fortran-77. Since its initial release, a number of upgrades in the user interface and in available tasks have been incorporated into the code by the Computer Research and Applications group at Los Alamos. Further planned enhancements are discussed below.

An outline of the tasks performed by TIPC is presented in Section II. A brief discussion of the physical processes inherent to transionospheric propagation of RF signals and the approximations incorporated into T!PC are presented in Section IJI. A brief statement outlining the tasks performed by each subroutine in TIPC is given in Section IV, while sample code runs with representative output are presented in Section V. A discussion of planned cuhancements to the code is given in Section VI.

\section{PROGRAM OUTLINE}

TIPC was designed to perform five basic tasks related to transionospheric propagation, the detection of RF signals following propagation and signal analysis of pulse waveforms, namely (1) transionospheric propagation. (2) signal filtering. (3) signal processing. (4) DTOA study, and (5) D'TOA mecertainty study. Task two ntilizes the output of one while task three uses the output of both one and two. and tasks four and five independently use the outputs of tasks one, two. and three. Several supporting calculations that allow the code to treat a bread range of problems of interest are performed

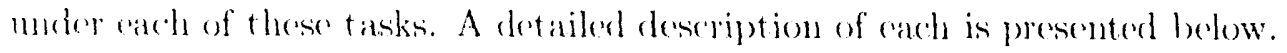

TlPe answmes that all input times are specified in microseconds and that the rorresponding frefueney information is given in $\mathrm{MH} \%$. In addition. the propagating clectromagnetic ficld is dofined in terms of its electric field component in mits of volts/moter. The Fourier transform of the signal has 
units of volts/meter. MHz. The output. of conrse, follows the same convention. Array sizes are fixed in a set of common blocks. Complex arrays with dimension 10.001 points are used to store information in the frequency domain while real arrays of the same dimension are used for corresponding data in the time domain. The restricted size of these arrays leads to inherent limitations on the problems that can be addressed by TIPC. These will be discussed below and in the following section. The default time resolution $\Delta t$ is taken to be $0.001 \mu$ with a $N y q u i s t$ frequency of $500 \mathrm{MHz}$. This choice provides a framework in which TIPC can address a broad range of problems of interest and at the same time maint ain reasomable execution times.

\section{A. Transionospheric Propagation}

In this section of the code a convolution of a specified signal with the impulse response function of the jonosphere is performed to obtain the transionospheric signal. This is accomplished hy taking the fast Fourier transform (FFT) of the input signal, multiplying against the transfer function of the ionosphere (defined in the following section), and taking the inverse transform of the product to yielel the transionospheric signal. Thus, we have

$$
E_{T I}(t)=(1 / 2 \pi) \int E(\omega) H_{I}(\omega) \exp (i \omega t) d \omega
$$

where $E_{T I}$ is the transionospheric signal. $E(\omega)$ is the Fourier transform of the input signal $E(t)$, and $H_{1}$ is the transfer function of the ionosphere. Equation (1) is evaluated numerically by means of an inverse FFT.

Ciearly, the first step in this process is the specification of an input signal. The code provides for a number of optional forms, including (1) Delta function. This function is created in the time domain by assigning an amplitude equal to $1 / \Delta t(\mathrm{~V} / \mathrm{m})$, where $\Delta t$ is the specified time step for the problem, at a single point. The resulting FFT has a flat spectrum with amplitnde mity $(\mathrm{V} / \mathrm{m} \cdot \mathrm{MHz})$. In general it is possible to specify an input pulse that consists of many delta functions separated by specified amounts in time. The default case is a single delta function placed at $t=4,000 \Delta t$. This functiona! form is the simplest representation of an impulsive signal that can be nsed to ducidate the offects of transionospheric propagation on RF signals. It can also be nsed to check the accuracy of the mumerical results by comparing against analytic forms for the impulse response function of the ionosphere. (2) Double exponential function. This form has been adopted as a standard for representing nuclear generated EMP and can be written analytically as

$$
E(t)=\frac{E_{m}\left(\exp \left[-C\left(t-t_{0}\right)\right]-\exp \left[-D\left(t-t_{1}\right)\right]\right)}{(C / D) ! C /(C-D)]-(C / D) ! D /(C \cdot D)]}
$$

where $E$ is the electric field strength in $\mathrm{V} / \mathrm{m} ; E_{m}$ is the maximum electric ficld occurring at $t_{m}=$ $t_{0}+\ln \left((1 D) /(C-D): t \geq t_{0}\right.$, where $t_{0}$ is the starting time for the pulse; $C<D ;$ and $\left.1 /(D)+C\right)$ is the rise time of the pulse and $1 / C$ the decay time. The de fault parameters used in PIPC are $C=8()$ MHz. $D=100 \mathrm{MHz} . E_{m}=500 \mathrm{~V} / \mathrm{m}$, and $t_{0}=0.5 \mu \mathrm{s}$. (3) Super Gaussian function. This functional form can be used to model symmetric pulses with fiat tops and fast rise and decay tines. The analytic representation is writen:

$$
\left.\left.E(t)=E_{m} \times x\right)\left[-\mid\left(t-t_{0}\right) / \Delta t\right]^{n}\right]
$$


where $n$ is even and effectively defines the shape of the function. The higher values of $n$ correspond to flatter tops with faster rise and decay times. The code also allows for five reflections, with specified delays and relative amplitudes, to be added to the direct signal. The default parameters for the direct pulse are $E_{m}=1 \mathrm{~V} / \mathrm{m} . t_{11}=0.03 \mu \mathrm{s} . \Delta \tau=0.004 \mu \mathrm{s}$ and $n=4$. Two reffection components are also added. with parameters $E_{m}=0.3 \mathrm{~V} / \mathrm{m}, t_{0}=0.04 \mu \mathrm{s} . \Delta \tau=0.004 \mu \mathrm{s}, n=4$, and $E_{m}=-0.2 \mathrm{~V} / \mathrm{m}$, $t_{0}=0.05 \mu \mathrm{s}, \Delta \tau=0.004 \mu \mathrm{s}, n=4$. (4) Modified Gaussian function. This form has been used to represent the EMP expected from a CPB device. The analytic form can be written:

with

$$
\begin{aligned}
& E(t)=\beta I_{n+t} \sin \theta /[(1-\beta \cos \theta) R], \\
& I_{n \cdot t}=I_{0}\left\{\frac{1}{(1+\operatorname{cox})\left[a_{0}\left(t-t_{u^{\prime}}\right) / t_{f}\right]}-\frac{1}{1+\exp \left[\frac{a_{0} t}{t_{r}}\right]}\right\},
\end{aligned}
$$

and where 3 is the beam velocity divided by the speed of light, $\theta$ is the polar angle of the observer off the bean axis. $R$ is the direct distance from beam origin to observer in meters, $I_{0}$ is the initial beam cursent at exit in $\mathrm{kA}, t_{r}$ is the current $10 \%$ to $90 \%$ rise time in $\mu s, t_{f}$ is the current $90 \%$ to $10 \%$ decay time in $\mu$ s. $t_{u}$ is the pulse width (FWHM) in $\mu$ s, and an has a ixed value of 4.4 . The code also allows for up to five reflections with specified delays and relative amplitudes to be added to the direct signal. The default parameters for this case are $I_{0}=10 \mathrm{kA}, t_{r}=0.01 \mu \mathrm{s}, t_{f}=0.02 \mu \mathrm{s}, t_{w}=0.05 \mu \mathrm{s}, \theta=90$ degrees, $r=0.999$. and $R=250.000 \mathrm{~m}$. The default pulse does not include reflections. (5) Specified input pulse. This option allows the user to designate an input file containing an arbitrary shaped pulse. The first line of the file specifies the format in which the subseguent amplitude and time information will be read (see the TIPC User's Manmal for further details). The pulse is read in and interpolated onto the appropriate grid.

As discussed in the following section, in the alsence of birefringent effects introduced by the geomagnetic field and to first order. the ionosphere can be specified in terms of the total electron content (TEC, $\equiv \int n_{c} d s$ ) along the line of sight and its primary effect is to disperse the input signal in tine, i.e., the ionosphere introduces a frequency-dependent delay in the arrival of different frequency components. These delays are sufficiently large relative to the duration of signals of interest that the individual frecuency components become resolvable in time, with the high frequencies arriving first. Near the cutoff frequency of the ionosphere (maximum plasma frequency), the time delays rapidly approach infinity. This sets an absolute lower limit on the frequency that can be modeled computationally. with a practical lower limit being somewhat above cutoff depending on the particular ionospheric model (i.e. total electron content). The user is therefore asked to specify a particular frequency range of interest. When the time difference of arrival between the upper and lower frequencies exceeds the length of the initial time window, the user is asked to supply a new value for the lower frequency such that the entire frequency lange fits into the time window (the minimum frequency allowed by the time window is computed and displayed for the user). A filtering procedure is then implemented in the frequency domain so as to avoid aliasing in the time domain. The filtering in the frequency domain is accomplished with a functional form that is flat with amplitude unity over most of the frequency range and falls off exponentially at both the high- and low-frequency ends with two separate exponential forms that are forced to match at the $50 \%$ transmission point. This functional form. shown in Figure 1. preserves the signal amplitude through most of the time domain and minimizes ringing near the edges of the window. Clearly, there exists a tradeoff between the latter effert and aliasing. These problems can be avoided by introducing much larger arrays and versions of 'TlPC with dimensions of 100,001 points have been written. Unfortunately the cost in processing time is significant [proportional to dimension $\log$ (dimension)]. 
TIPC also treats the birefringence of the ionosiphere. In this case the nser is asked to specify the percent of extraordinary mode to be mixed in with the ordinary mode (0\% the defante corresponds to a pure ordinary wave) in the transionospheric signal, and to identify the hemisphere in which prepagat ion will occur.

In addition to a deterministic component associated with the mean vertical structure of the ionosphere and parameterized in terms of the total electron content, the impulse response function in general also includes a statistical component produced by the large, random horizontal density gradients observed in the ionosphere and paraneterized in terms of a frequency coherence bandwidth. The user is given the option of including this effect and asked to input a value for the frecpuency coherence bandwidth of the ionosphere. $U_{P}$ to 25 realizations of the impulse response function (IRF) and corresponding transfer function are generated in the replacement algorithm for CIRF. In the case of transionospheric propagation, a single realization is selected and convolved against the deterministic response function to obtain the tinal transionospherie signal. This procedure can be represented mathematically by simply redefining the ionospheric transfer function in equation (1) to be

$$
H_{l}(\omega)=H_{D}(\omega) H_{S}(\omega)
$$

where $H_{D}(\omega)$ is the deterministic part and $H_{S}(\omega)$ is a single realization of the scattering part. both of which are defined in the following section.

In addition to specifying the propagation chamel, the user is also given the option of adding Gaussian-distributed white noise to the input signal. The amplitude of the noise is determined from the signal-to-noise ratio (SNR) specified by the user and the peak signal power, i.e.,

$$
P_{N}=E_{p}^{2} / S N R
$$

where $E_{p}$ is the signal peak amplitude and $P_{N^{\prime}}$ is the noise power (standard deviation 'squared of Gaussian distribution). The noise, however, is actually added to the signal in the frequency domain. Thus, the noise spectrum is defined as

$$
E_{N}(\omega)=r_{1}+i r_{2}
$$

where $r_{1}$ and $r_{2}$ are two independent random variables obtained for each frequency value from a Gaussian distribution with standard deviation squared given by

$$
\sigma_{N}^{2}=E_{p}^{2} T \Delta t / S N R
$$

where $T$ is the length of the time window and $\Delta t$ is the sampling time interval. $E_{N}$ is simply adcled to the FFT of the input signal, the result multiplied by the transfer function of the jonosplere and an inverse FFT performed [see equation (1)] to obtain the "noisy" transionospheric signal.

TIPC also allows for "colored" noise. In this case the noise amplitude $\left(\sigma_{N}\right)$ as a function of frecuency is defined by the user and read from a specified input file. The input consists of a header indicating the number of points and format of the data followed by a tabular list of freculencies in MH/. RMS amplitudes of the noise in volts/meter. $\mathrm{NH} z$ and standard deviations of the noise anplitudes in volts/meter. $\mathrm{MHz}_{\mathrm{z}}$ (see the TIPC User:s Mamual for further information). The noise is then adderl to the signal in the same way as outlined above for white noise. Clearly, the particular noise distribution added to the input signal represents only one statistical realization that may or may not reflect the 
mean properties of the Gaussian distribution. This procedure takes on meaning only when a series of realizations of some measured quantity is obtained in order to characterize that quantity's statistical properties. This point will be discussed further in the section on D'TOA uncertainties.

The final output of this task is the transionospheric signal over a time span that incorporates the specified frequency range. In the presence of noise or scattering, the transionospheric signal represents only one random statistical realization of potential outcomes. The output takes on the form of plots and the user is given the option of displaying any or all of the following: the input pulse, input pulse spectrum, and the transionospheric signal.

\section{B. Signal Filtering}

Signal filtering in this context refers to a complex measurement of both the amplitude and phase of the transionospheric siginal over a specified bandwidth. The filtering process is simulated by convolving a given filter response against the transionospheric signal, yielding for the filtered transionospheric signal

$$
E_{F^{\prime} T I}(t)=(1 / 2 \pi) \int E(\omega) H_{I}(\omega) H_{F}(\omega) \exp (i \omega t) d \omega
$$

where $H_{F}(\omega)$ is the transfer function of the filter.

In the present version of TIPC the user is given the option of either specifying the filter transfer function, i.e., amplitude and phase (or real and imaginary parts; see TIPC User's Manual for details of implementation) as a function of frequency, or selecting from two possible filter shapes, namely a narrowband Gaussian filter parameterized in terms of its full width at half maximum (FWHM) and central frequency or a broadband filter parameterized in terms of the upper and lower frequency values at which the filter achieves $50 \%$ transmission. The broadband filter is characterized by a rapid rise in transmission (computed by means of two exponentials spliced together at the $50 \%$ transmission point) followed by unit transmission over most of the bandwidth and a rapid decay in transmission (computed in the sanc way as the rise). Up to some maximum (presently set to four, see the TIPC User's Manual) namber of filters can be specified for any given run. The default parameters for this task are a single Gaussian filter at $150 \mathrm{MHz}$ with a $2-\mathrm{MHz}$ bandwidth (FWHM).

The final output of this task is the filtered transionospheric signal. A number of plots can be displayed. The user can choose to have the filter profiles in the frequency domain and/or the filter response to a unit impulse in the time domain plotted. Similarly the filtered transionospheric signal can also be displayed in either the frequency or the time domain or both. TIPC also gives the user the option of plotting the power spectrum of the input pulse overlaid with the peak power measured by each of the receivers. The latter plot is particularly useful in indicating the utility of narrowband amplitude measurements in estimating the power spectrum of the original signal.

\section{Signal Processing}

Two levels of signal processing are implemented in TIPC. The first offers the choice of performing rither cluadrature detection or square-law detection of the signal. Quadrature detection simply refers to an algorithm that computes the in-phase (I) and $90^{\circ}$ out-of-phase (Q) components of the filtered signal and displays the results. Quite often this form of signal processing also requires beating the 
received signal against a local oscillator so that the signal can be digitized at a slower rate. The real, $90^{\circ}$ phase shifted component $P(t)$ of the filtered transionospheric signal is given by

$$
P(t)=(i / 2 \pi) \int_{0}^{\infty}\left[E_{F T l}(\omega) \exp (i \omega t)-E_{F T l}^{*}(\omega) \exp (-i \omega t)\right] d \omega
$$

The final I and $Q$ components beat down by a local oscillator of frecuency $F_{L O}$ are then

$$
I(t)=E_{F T I}(t) \cos \left(2 \pi F_{L O} t\right)-P(t) \sin \left(2 \pi F_{L, ~} t\right)
$$

and

$$
Q(t)=E_{F T I}(t) \sin \left(2 \pi F_{L O} t\right)+P(t) \cos \left(2 \pi F_{L O} t\right) .
$$

The code also computes the amplitude $\left[\equiv\left(I^{2}+Q^{2}\right)^{1 / 2} / \sqrt{ } 2\right]$ and the phase $\left[\equiv \tan ^{-1}(Q / I)\right]$ of the filtered transionospheric signal.

For square-law detection the envelope of the received signal in any given channel is obtained by scluaring the signal and passing it through a low-pass filter whose bandwidth is specified by the user and defaulted to $10 \mathrm{MHz}$. The shape of the low-pass filter is identical to that of the broadband filter described in Section II.B. Thus

$$
E_{S L}(t)=\left[(1 / 2 \pi) \int E_{s}(\omega) H_{L, 1}(\omega) \exp (i \omega t) d \omega\right]^{1 / 2},
$$

where $E_{s}(\omega)$ is the Fourier transform of the filtered transionospheric signal squared and $H_{L, p}(\omega)$ is the transfer function of the low-pass filter.

The second level of signal processing incorporates three different algorithms designed to extract the ionospheric TEC from the transionospheric signal. In general the TEC can be determined from the chirp rate of the signal (FM technique) or by simply measuring the difference in the time of arrival (TOA) of the signal at a minimum of two frequencies (AM techique). In the latter technique a method for specifying an arrival time for the signal in a given channel must be adopted. Although many different' mothods have been proposed, we have chosen to work with an algorithm in which the one-third and two-thirds amplitude (relative to the maximum) points on the leading edge of the envelope of the filtered signal are time-tagged to obtain a slope from which a TOA is computed by simple extrapolation to a zero-amplitude baseline. TIPC performs this calculation for each channel given the ontput envelope computed by the square-law detection algorithm. In the FN technicpue initially developed at Los Alamos National Lab by R. Massey (mupublished), zero (rossings of the filtered transionospheric signal are used to determine the period as a function of time (chirp rate of the signal) for each receiver. A corresponding TOA is obtained from the intercept of a straight-line fit to the measured period squared vs. time. This TOA represents the time of arrival of an infinite frequency component, i.e., the effects of the ionosphere are completely removed independent of the filter used. The third algorithm is designed to yield a delta-time-of-arrival (DTOA) between any two receivers. The output of square-law detection (signal envelope) for a given channel $(i)$ is convolved against that of another channel $(j)$ to yield a cross-correlation amplitude $\left[C_{i, j}(r)\right]$ as a function of the time separation $(\tau)$ between channels. Thus, we have

$$
C_{i, j}(\tau)=\int E_{S L L, i}(t) E_{S L L, j}(t-\tau) d t
$$

The time of the peak cross-correlation amplitude is computed and defined to be the D'T(OA for that pair of receivers. This process is repeated for all possible pairs. 
The final output of this task depends on the particular subtask selected. In the case of quadrature filtering, four plots consisting of the I-component, Q-component, amplitude, and phase of the transionospheric signal as a function of time are displayed for each channel. For square-law detection, the cnvelope of the transionospheric signal is plotted as a function of time. The output of the FMTOA subtask consists of plots of period squared vs. time for all receivers, while for the leading-edge TOA, a plot of the envelope of the filtered transionospheric signal is displayed for each channel. For the crosscorrelation subtask, a plot of the cross-correlation function vs. the time separation between channels is produced for cach pair of receivers.

\section{DTOA Study}

For this task the user is asked to specify a range of TECs over which to carry ont a series of TOA measurements. TIPC then automatically loops through the first three tasks, given the selected settings for each, and accumulates DTOAs for all possible pairs of chamnels. 'The user must of course specify a TOA method (FMTOA or Leading-Edge) or the cross-correlation algorithm in signal processing to generate the DTOAs. The DTOAs obtained with any of the selected methods are plotted vs. TEC for all charmel pairs. In this way the user can generate a look-up table that yields the actual TEC for a given DTOA measurement for any combination of time-tagging algorithm and system of receivers. A rough estimate of the TEC can be obtained from the DTOA directly, independent of the look-up table, with the formula

$$
T E C=2 \pi D^{\prime} O A /\left(\beta_{1} / f_{1}^{2}-\beta_{2} / f_{2}^{2}\right)
$$

where TEC is in mits of $10^{13} \mathrm{~cm}^{-2}$. DTOA is in $\mu \mathrm{s}, f_{1}$ and $f_{2}$ are the central frequencies of the two channels in $\mathrm{MHz}$, and the $\beta$ 's are defined as

$$
\beta_{1,2}=8.430 \times 10^{4} /\left(1 \pm 0.8 \sin \theta, / f_{1,2}\right)
$$

where $\theta$ is the angle between the wave propagation direction and the geomagnetic field. The FMTOA method represents a special case because in principle the effects of the ionosphere are completely removed for any individual channel. The DTOA should therefore be zero or equal to the difference in receiver delays for any pair of channels. Deviations from these values are an indication of the errors incurred by the particular system under study with the FMTOA technique. The final output of this task is a plot of D'TOA vs. TEC for cach pair of chamnels.

\section{E. DTOA Uncertainty Study}

This task allows the user to study the effect of Gaussian white noise or of a structured ionosphere on the ability of a specified algorithm and system of receivers to determine the ionospheric TEC. The user is asked to specify a range of signal-to-noise ratios or frecuency coherence bandwidths over which the study is to be performed. TIPC then loops through tasks one through three, accumulating DTOAs for all pairs of receivers and for up to 25 realizations of each of up to 5 SNRs or frequency coherence bandwidths. The root-mean-square deviation from! the mean for the DTOAs as well as the mean are computed and plotted vs. SNR or coherence bandwidth. In this way the user is able to estimate a sperified system's ability to determine TeC moler noisy or disturbed conditions. 


\section{IONOSPHERIC MODEL}

Among the effects associated with the propagation of electromagnetic waves through a plasma alre dispersion, reftection, refraction, absorption, and scattering. We discuss cach of these processes and indicate which are treated in the TIPC ionospheric model.

\section{A. Dispersion}

The dispersion of an electromagnetic pulse (EMP) incident on a plasma ariscs becanse we hifferent. frequency components which make up the temporal structure of the pulse propagate at. differen: speeds defined by the index of refraction of the plasma. The presence of the earth s magnctic ficld also makes the ionosphere a birefringent medium, separating an incident pulse into two modes (ordinary and extraordinary, corresponding ronghly to right-hand and left-hand polarization, respectively, in the northern hemisphere), each propagating at a different speed. For the ionosphere the index of refraction is given by the Appleton-Hartree formula (see e.g., Rishbeth and Garriot, 1969; Davies, 1965), which in the limit where the electron collision frequency is small compared to the frequencies of interest reduces to

$$
\mu^{2}=1-\frac{X(1-X)}{(1-X)-Y_{T}^{2} / 2 \pm\left[Y_{T}^{4} / 4+(1-X)^{2} Y_{L}^{2}\right]^{1 / 2}},
$$

where $X=\omega_{p}^{2} / \omega^{2}, Y=\omega_{H} / \omega, Y_{T}=Y \cos \theta, Y_{L}=Y \sin \theta$, and $\theta$ is the angle between the wave propagation direction and the geomagnetic field. The plus (minus) sign in equation (18) corresponds to the ordinary (extraordinary) mode. In the limit $Y_{T}^{4} / 4<<(1-X)^{2} Y_{L}^{2}$ (quasi-longitudinal approximation), this equation further simplifies to

$$
\mu^{2}=1-X /\left(1 \pm Y_{L}\right)
$$

Assuming that $Y_{L}$ is small, the group velocity can be written

$$
V g=c\left[1-X /\left(1 \pm Y_{L}\right)\right]^{1 / 2}
$$

As is evident from equations (18) (20), the net effect of slispersion is to introduce a time delay in the arrival of a particular frequency component of the incident, pulse at the detector such that the high frequencies arrive first.

The transionospheric signal can be obtained for arbitrary electromagnetic pulses by convolving the pulse time history against the impulse response function of the ionosphere. Alternatively, onc can multiply the Fourier transform of the pulse against the transfer function of the ionosphere. 'The. latter can be derived from the equations given above for the index of refraction. In the limit that the quasi-longitudinal approximation applies and when $X<<1$, the transfer function of the deterninistic ionosphere can be written

$$
H_{i}(\omega)=\exp (i 2 \pi \alpha / \omega)
$$

where $\alpha=8.430 \times 10^{4} \mathrm{TEC} /\left(1 \pm Y_{L}^{*}\right)$, TEC is the total electron content along the propagation path in units of $10^{13} \mathrm{~cm}^{-2}, Y_{L}^{*}$ is taken to be $0.8 \sin \theta / f$, and $f$ is the frecuency in MHz. Clearly, the choice of $0.8 \sin \theta / f$ for $Y_{L}^{*}$ assumes some weighted magnetic field strensth and angle between the propagation vector and the magnetic field. The particular choice $\left(f_{n}=0.8 \mathrm{MHz}\right)$ is typical of micllatiturle prop)agation: however, because of the density weighting along the propagation path it is also possible to derive a more accurate value that depends on the latitude and longitude of the soures and the arimuth and elevation angles of the receiver. The liater algorithm is not presently implenonted in TIPC. The 
angle $\theta$ can be determined from the magnetic dip angle at the altitude of peak ionospheric density and the propagation direction.

\section{B. Reflection}

Reflection ocenes for the frecuency components of the incident pulse that are below the peak plasma frequency. For a culesecent ionosphere, the plasma frequency generally falls below $9 \mathrm{MHz}$. As a result, frecuency components at and below this value never arrive at a transionospherie receiver. Those frequencies just above the plasma frequency are strongly dispersed and refracted by the ionosphere and are therefore difficult to measure and interpret. Becanse the ionospherie model incorporated into TIPC ansumes that the EMP frequencies of interest are much greater than the plasma frequency, and given the linited time window used by the code, frequencies below approximately $25 \mathrm{MHz}$ are not. well morkeded for an ionosphere with a peak plasma frecpuency of 9 MHz. TIPC accounts for reftection and strong dispersion in effect by filtering out frecunencies below that specitied by the user. It is, therefore, important to choose a lower frecunency limit that is approximately three times the peak ionospheric plasma frecuency in order to obtain an accurate transionospheric signal.

\section{Refraction}

Refraction is a process whereby the direction of propagation of a wave front is altered as a result of a change in the index of refraction of the medium. Refraction has the effect of increasing the path length from source to receiver, thereby increasing the TEC and the corresponding time delay. This effect, however. is second order in the parameter $X\left(=\omega_{p}^{2} / \omega^{2}\right)$ and is negligible at VHF frequencies, proviried the propagation vector is not at grazing incidence on the ionosphere. We note that the general concept, of refraction is applicable only in cases where geometrical optics accurately describes the propagation of the electromagnetic wave. When scattering processes dominate, interference phenomena become important and the simple ideas of geometrical optics break down.

\section{Absorption}

Absorption of the incident pulse occurs when accelerated eiectrons transfer their energy to ions and neutrals by collision. In the ambient ionosphere the collision time is large compared to the duration of the EMP and alsorption is negligible. In strategic environments, however, enharced densities lead to higher collision rates and correspondingly higher absorption. Althongh this effect is included in the general Appleton-Hartree equation, it is not treated in TIPC.

\section{E. Scattering}

Soattering is the process by which the propagation path of different parts of an incident wave front are redirected in random directions by the presence of local inhomogeneities or electron-density perturbations. The net result is an interference phenomenon in which random variations in the anplitude and phase of the incident wave, called scintillations, occur. Two important and distinct effects associated with severe scintillations are spatial variations in signal strength, which appear as dece fades 10 moving communications receivers, and temporal distortions in received signals of broad bandwidth systems, resulting from a mixing of different frecuency components of the melisturbed signal. The 
former effect has been studied extensively both experimentally with varions communications systems (Aarons, Whitney, and Allen, 1971; Uneki, Lin, and Yoh, 1977; Nartin and Aarons, 1977; Fremonw et al., 1978; Basu and Kelley, 1979; Basu et al., 1980); Livingston, 1980); Fremouw, Livingston, and Miller, 1980; Rino and Owen, 1980); Whitney et al., 1981: Rino et al., 1981a and b; Johnson, 1981; Basu et al. 1983; Kelley et al. 1985) and theoretically (Booker, Ratcliffe, and Sherin, 1950); Thatarskii, 1971a or b; Budden, 1965: Salpeter, 1967; Lowelace et al., 197(); Barabanenkov et al., 1971; Rufenach, 1975a and b; Bahar and Agrawal, 1976) and is generally characterized in terms of the $S_{4}$ scintillation index, which is a measure of the root-mean-square deviation in signai intensity.

The extent of temporal distortions resulting from propagntion through a structured atmesphere has been studied both theoretically and experinentally only in the last ton years (Rufenach, 1975a and b:

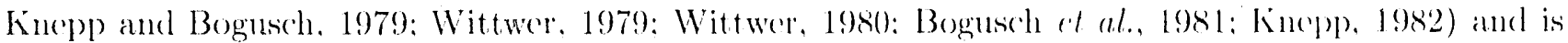
generally characterized in terms of Foh, the frecpueney coherence bandwidth of the medium. The imparet of scintillations on received signals is to introduce adelitional uncertainties in signal measurement and time-tagging because of reductions in SNR from fanding and distortions in the temporal structure of the signal. The magnitude of these effects is random and can only be characterized statistienlly. As a result. one cannot compensate for them but must include them in assessing a system so performance.

The theoretical treatment of the scattering of radio waves in the ionosplere proceeds from the parabolic wave equation. which can be solved in its most general form by the use of multiple phase screen (MPS) technicues (see c.g.. Knepp, 1982). In the limit of strong scattering conditions, the assumption of a quadratic phase structure function and the thin phase screen approximation combine to greatly simplify the and lysis, yielding an analytic solution for the two-position, two-frecfucney mutual coherenee function from which the inpulse response function (IRF) can be derived. Physically the IRF represents the signal that would be measured following propagation of a delta function (in time) radio pulse through a thin phase screen. When the IRF is convolved against the mean dispersive effects of the ionosphere and against a given radio pulse, the result is a particular signal realization that is associated with the selected phase screen. Propagation through appropriately chosen phatse screens then yiolds a statistical set of signal realizations from which the accuracies of 'T')A measurements, for example, ("an be determined. In the linit of a thin phase screen and assuming an isotropic distrihution of irregularities, it is possible to represent the average statistical onteme of a series of such experinents by means of the power impulse response function (representing an average of many statistical realizations of the IRF s(plared), which can be written (see Knepp 1982, p. 175)

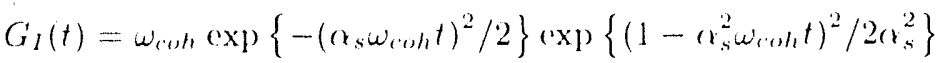

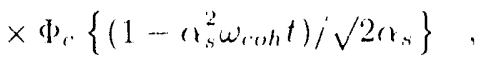

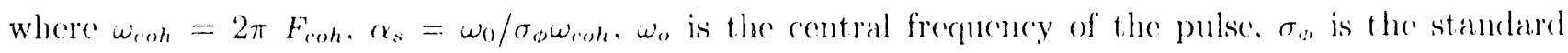
deviation of phase fluctuations as defined bolow, and $\Phi$, is the complementary cror function. (i,

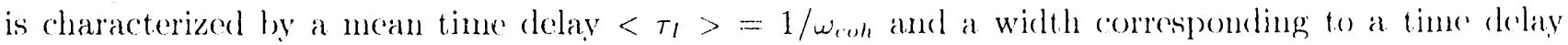
jitter $\sigma_{I}^{2}=\left(1+1 / \sigma_{s}^{2}\right) / w_{r o h}^{2}$. In this approximation the statistical properties of the phase screens are

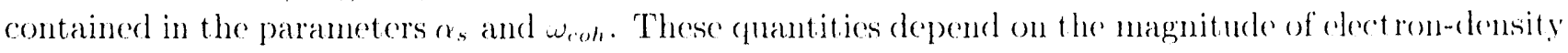
perturbations and their associated seale sizes in a manner defined below. A single stat istical realizat ion of the scattered signal, on the other hatnd, is written

$$
E_{S}(t)=\left\{\left(i_{1}(t) / \Delta t\right\}^{1 / 2} r .\right.
$$


where $r\left(=\left(r_{3}+i r_{2}\right) / \sqrt{ } 2\right)$ is a complex mumber whose aeal $(r)$ and inaginary $(r, 2)$ parts are independent Gaussian random variables with zero mean and mity variance. The scattering transfer function $H_{s}$ (w) for use in equations (6) and (1) is obtained by taking the Fourier transform of equation (23).

The phatse sereens needed in the above analysis are generated from the known magnitude of electrondensity fluctuations and their corresponding spectrum of seale sizes. Assuming the latter distribution to be isotropic and a power law in $k$-space $\left(k^{*}\right.$ in three dinensions). one can derive three important propagation paraneters in terms of the RMS electron-density fluctuations $\left(\sigma_{m}\right)$. inner and outer scale

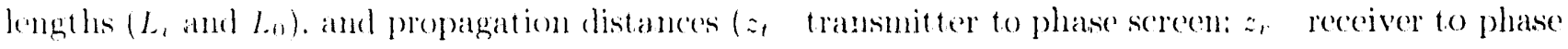
screc(11). mandely

$$
\begin{aligned}
& \sigma_{\omega^{\prime}}=\left(2 I_{,} l_{n(1)}\right)^{1 / 2} r_{1} \lambda_{0} \sigma_{n} .
\end{aligned}
$$

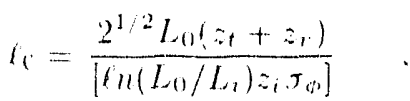

and

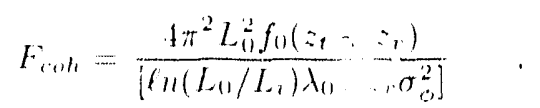

where $"$ is the (lassical electron ranlius $\left(=2.82 \times 10^{-13}\right.$ ('m) $) \lambda_{10}$ and $f_{0}$ are the central wavelength and frepuency. respeetively. of the electromagnet ie wave: $L$ is the thickness of the phase screen: $\sigma_{0}$ represents the RMS phase fluctuation, Fon is the frecueney coherence bandwidth: and $l_{0}$ is the sigatial correlation length associated with scintillations. The parancters together with the sis scintillation index deseribe the effects of strong sicattering on a radio pulse propagated through a structured ionosphere.

It is inportant to note that the above formalism applies only to a narrowband radio pulse with a carrier frepuency. fo. that is well absere the plastna frequency. In addition. it is evident from equations (2.4) (26) that the intportant propagation parameters depend rather strongly on the carrier frequency (e.g.. F . . . $\left.f_{10}^{4}\right)$. making it difficult to apply this method of analysis to a broatband pulse. The most. gemeral form of multiple-phase-seresn terhuigues is necessary to treat the problem of broadband-pulse propagation through a structured plasma.

Nevertheless. fo calculate the temporal distort jons int reduced by propagation through a structured

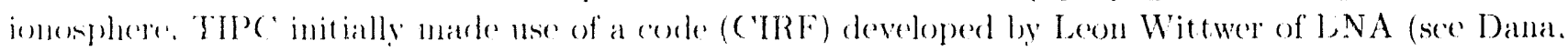

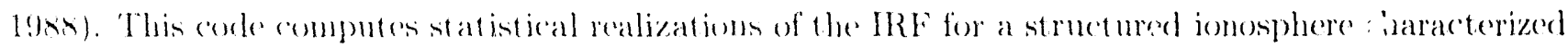
by isotropje irregularition with a 1-I) power-law spectral density profile with spectral index of two. The only iuput to ('IRF is Fins all other propagat ion parameters are defanlted to values that correspond

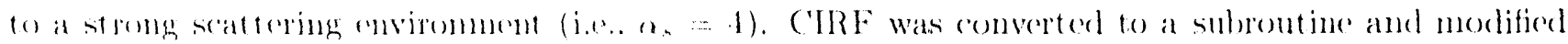
slightly or return the Fonder transform of a single realization of the IRF. More recently, we have implemented a new algenthom that makes use of equation (23) to generate statistical realizations of the impulse reponse function. In onr new algorithm. we ignere the spatial effects of scaftering (atsiuning the recevere in stationaty eser the time duration of the sagnal) and replace the generalized power

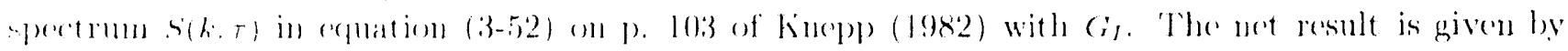

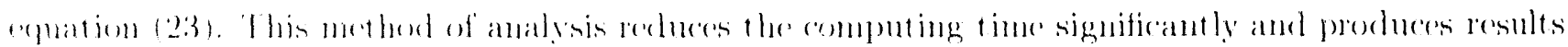

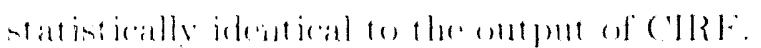

Becallse the coherence banderidth is assmued to be a constant across the spectrum of the EMP. the transionesplerie signal computed in this way shomld be considered only as indicative of the effects

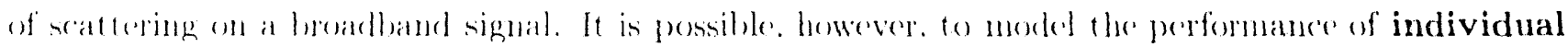


natrowband receivers precisely by choosing the appropriate value for the coledence bandwidth conore-

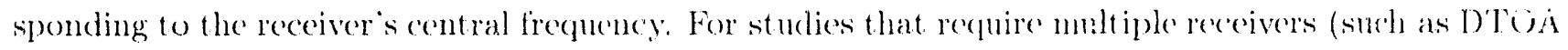
studies). however, the coherence bandwidth is treated as a constant.

To complete the analysis of pulse propagation through a structured enviromonent. it is alse nocessiry to consider the inpact of signal fades associated with amplitude and phase scintill ations. This can be acomplished with TIPC by simply varying the SNR in a way commensurate with the statistical amplitude variations to be expected for the desired s. scintillation index.

\section{F. Deterministic Ionosphere}

The vertical TEC of the ionosphere can vary by more than an order of magnitude depending on the latitude. longitude. solar activity. season. and time of day. It is important. there cone o late as accumate an estimate of TEC as possible for the particular propagation chamel of interest. The Juternational Reference Ionosphere (IRI) (Rawer et al. 1978a anci b: Bilitza. 1986; Rawer and Bradley. 1987) is a computer program that generates vertical profiles of electron density. electron and ion temperatures. and positive ion composition for any given geogr phical location. time of day, seasom. and solar activity. The electron-density profile is computed by interpolating a series of tables developed from sis reptic meastrements obtained with ionosondes. topside someders. and incoherene scatter radars. Ty, deita batse in some cases constitutes more than 20 years of moasmenents taken on an hourly basis.

The input parameters to IRI include the geographical location (latitude and east longitude). the sumspot mumber (avalable from the Geomannetio and Solar Data published monthly by the fournal of Ceophysical Rescarch). the month. time of day (hour). solar inclination. lower and upper limits of the height profile and the profile height resolution. The ontput includes plots and a listing of the ionospheric const it uents and electron and ion temperatures as a function of height. The clectron-density profile can then be integrated to vield a 'TEC for input to 'TIPC.

\section{G. Structured Ionosphere}

The existence of electron-edensty irregularities in the jonosphere has heen known for some time (see. e.g. Fejer and kelley 1980) and their variability and effect on radio commenieations has been studied extensively as noted above. Concrally. the largest electron-density perturbations are fommol both at high latitule and at the equate" and are associated with magnet ic subtorms and corresponding alectron precipitation in the anroral owal and with equatorial spread-F. In contrast. midlatitude fluctuations

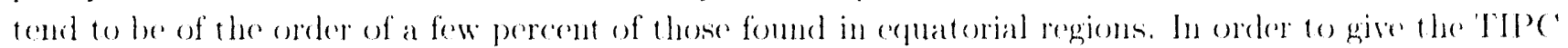
user a ferling for the magnitude of $s_{4}$ and Fon. We present a brief discussion of some of the available

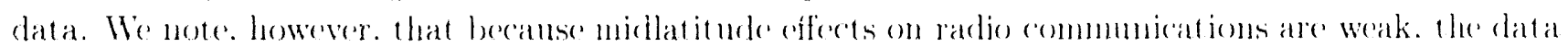
avalable for this region are meager at best and our discussion is therefore limited to the werst-atse comblitions found in polar and equatorial regions.

The magnitude of ionospherie density fhetuations is either inferred from scintillations in radio sigmals or measured in sitn. The largest RMS density perturbations range from $1: 3 \times 10^{5}$ ('m ${ }^{3}$. while the

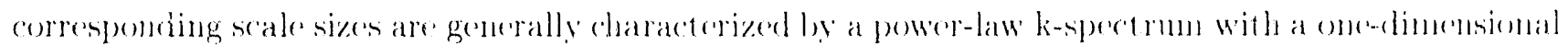

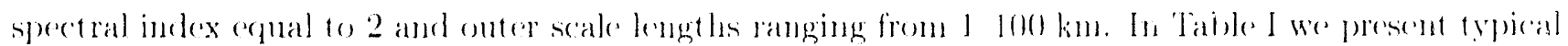

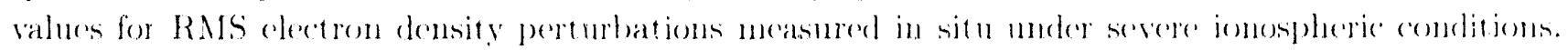
along with the eoresponding propagation paraneters. The latter paraneters were calculated assuming

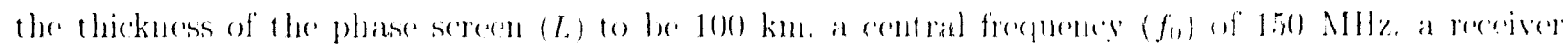


distance $\left(z_{r}\right)$ equal to $20.000 \mathrm{~km}$, a source distance $\left(z_{t}\right)$ of $300 \mathrm{~km}$, and an inner scale length equal to $200 \mathrm{~m}$. Note that the inner sate length was chosen somewhat arbitrarily. repre ienting the resolution of in-situ measurements in some cases, but that the propagation parameters do not depend strongly on $L_{i}$. The ion density measurements of Phelps and Sagalyn (1976) were obtained with the spherical edectrostatic analyzer probe on ISIS 1 in the topside ionosphere at high latitudes (60) 8.4 degrees invariant latitud(e) and altitudes ranging from 574 to $3523 \mathrm{~km}$. Irregularity scale sizes ranging from 200$) \mathrm{m}$ to $100 \mathrm{~km}$ and irregularity amplitudes as high as $20 \%$ of the mean were measured. Assuming that these characteristics persist throughout the ionosphere, one would denive a relevant electron-rlensity perturbation of $10^{5} \mathrm{~cm}^{-3}$ and the corresponding propagation parameters shown in Table I. Oyson. McClure, and Hanson (1974) report on similar results obtained with the retarding potential ankilyzer on OGO-6. These measurements included equatorial regions, which weic found to possess somewhat higher irregularities than the polar regions. The rocket measurenents of Costa and kelley (1976) were taken in the bottomside ionosphere (altitudes of $27($ ) $340 \mathrm{~km}$ ) over Natal. Brazil. with a Langmuir probe. The results presented in Table I for these measurements are representative of a density hole or plunclike structure near the F-region peak. The OGO-6 measurements reported by Basu, Basu, and Kahn (1976) were obtained with the retarding potential analyzer over various equatorial regions at an altitude of $400 \mathrm{~km}$. The irregularity amplitude of $3 \times 10^{5}\left(\mathrm{~cm}^{-3}\right.$ is asscciated with severe ionospheric conditions which are common over the African and Atlantic sectors.

TABLE I

In Situ Measurements of Ionospheric Irregularities

\begin{tabular}{|c|c|c|c|c|c|}
\hline Ionospheric Conditions & $\begin{array}{c}\mathrm{L}_{o} \\
(\mathrm{kmin})\end{array}$ & $\begin{array}{c}\sigma_{u} \\
\left(\mathrm{~cm}^{-3}\right)\end{array}$ & $\begin{array}{c}\sigma_{\phi} \\
\text { (Radlians) }\end{array}$ & $\begin{array}{c}\ell_{0} \\
(\mathrm{~km})\end{array}$ & $\begin{array}{l}F_{1} \text { wh } \\
\left(\mathrm{MHz}_{\mathrm{H}}\right)\end{array}$ \\
\hline $\left.\begin{array}{l}\text { Topside. high-latitude" } \\
\text { Topsicle. equatorial" }\end{array}\right\}$ & 10 & $10^{5}$ & 25 & 20 & 63 \\
\hline Bottomside. (equatorial spread F" & 10 & $2 \times 10^{5}$ & 50 & 75 & 16 \\
\hline Near density poak, equatoriald & 2 & $3 \times 10^{5}$ & 34 & 3.7 & 2.4 \\
\hline $\begin{array}{l}\text { "Phelps and Sagalyn (1976). } \\
\text { "Dyson of al. (197t). } \\
\text { "Costa and Kelley (1976). } \\
\text { "Basu et al. (1976). }\end{array}$ & & & & & \\
\hline
\end{tabular}

Cnder strong scattering conditions, the existence of a scintillation index approxinately equal to one and corresponding signal fades of 20 to 30 dB at VHF frequencies is quite common. RMS phase fluctuations in the tens of radians at VHF are also typical. In Table ll. the results of two representative scintillation studies are summarized. Fremonw a al. 1978 report on scintillation measurenents taken

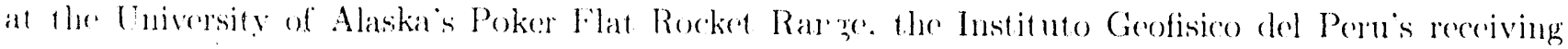
site at Ancon. and a receiving station at Kwajalein. The signats originated from a multifrecunency (spanning VHF to S band) coherent radio beacon on the DNA Wideband satcllite. At VHF (138 MH\%) the measurements are chatacterized by sighal fades as deepe as 40 dib and phase excursions of several

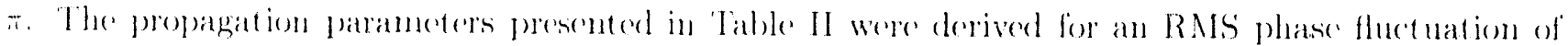


TABLE II

VHF Scintillation Measurements

\begin{tabular}{|c|c|c|c|c|c|}
\hline Ionospherie Conditions & $\begin{array}{l}\mathrm{L}_{1,} \\
(\mathrm{~km})\end{array}$ & $\left.\left(\mathrm{cm}^{\prime \prime}\right)^{-3}\right)$ & $\begin{array}{c}\sigma_{\phi} \\
\text { (Radians) }\end{array}$ & $\begin{array}{c}\ell_{0} \\
(\mathrm{k} m)\end{array}$ & $\begin{array}{l}\mathrm{F}_{\mathrm{cuh}} \\
(\mathrm{MHz})\end{array}$ \\
\hline $\begin{array}{l}\text { Polar (Poker Flat) } \\
\text { and equatorial (Ancon)" }\end{array}$ & 1,6 & $10^{5}$ & 10 & 10 & 19 \\
\hline Equatorial (Ascension Island) $)^{b}$ & 1.0 & $10^{5}$ & 8.0 & 9.3 & 11 \\
\hline
\end{tabular}

10 radians and assuming an RNS electron density variation of $10^{5} \mathrm{~cm}^{-3}$. 'The scintillation study of Rine et al.. (1981a) is based on moasurements taken on Ascension Island. which is located in the Sonth Athastic ionospherie equatorial anomaly region where strong scintillation offects are normally (o)served. VHF (250) MHz) signals originating from the Marisat satellite showed decp fartes from 20) 30 (B). Assuming an RAS electron-density fluctuation of $10^{5}$ ( $\mathrm{m}{ }^{3}$ and a magnitude of $10^{21}$ for their parameter $C_{3}$. one obtains an onter scale length of 1 km from their Figure IV-11. These values can be used to derive the propagation paraneters presented in Table II.

These results indicate that under worst-case ionospheric conditions, VHF signals will experience (leep fanles ranging from 20 to 40 (B and strong distortions in theije temporal structure for systems with bandwidths greater than approximately $2 \mathrm{MHz}$

\section{TIPC SUBROUTINES}

Nany of the subroutines used in TIPC are available from the standard Fortran math library. On the other hand. some of the rontines (e.g., FFT routines) were extracted from CLAMS and others were developed specifically for TIPC. A brief description of the individual routines and their associated input/ontput is given below. A chart showing the calling sequence and detailed nesting of these calls is given in Appendix A. The general flow of the program is illustrated in Figure 2.

AMAXMIN (ARRAY. N. AMAX. IMAX. AMIN. IMIN)

This subrontine searches the first $N$ elements of the array ARRAY for the maximmm and minimum value. It retums the maximum value in $\mathrm{AMAX}$ and its corresponding index in IMAX. It also returns the minimum value in AMIN and its corresponding index in IMIN.

AVGTINE (ARRAY. TAVG)

This subroutine uses the values in ARRAY as weights in constructing the weighted average of the times in TIME. The weighted average is returned in TAVG. NP'TS are used in the average. 
CFFTB $(N, C$. WSAVE $)$

This subroutine in FFT.FOR computes the backward complex (discrete) Fourier transform of the sequence of $N$ points in the array $C$. WSAVE is a work array that must be first initialized with a call to CFFTI.

\section{CFFTF $(N, C$, WSAVE)}

This subroutine in FFT.FOR computes the forward complex (discrete) Fourier transform of the seculuence of $N$ points in the array $C$. WSAVE is a work array that must be first initialized with a call to CFFTI.

\section{CFFTI $(N$. WSAVE)}

This subroutine in FFT.FOR initializes values in the work array WSAVE for use with CFFTB and (FFTF.

CIRF (CDT, DD, N'TIM, CR)

This subroutine computes NTIM values of a random function in the time domain, which is meant to simulate the stochast ic features of the ionosphere. The Fourier transform of this function is returned

in CR. CDT is the time increment to be used, while DD is the delay interval length to be used. The coherence frequency bandwidth, $F_{c o h}$, is also used in constructing the function.

\section{(LEAR (T'TY)}

This subroutine clears the user's screen and repositions the cursor. T'T'Y indieates the type of terminal in use. Two types of terminals are supported by this routine: DEC-compatible and Tektronixcompatible terminals.

\section{('ROSCOR (INDEX)}

This subroutine estimates the difference in times of arrivals for pairs of receivers by determining where the integral of $T i(t) T j(t-s)$ over $t$ is maximized ( $T i$ and $T j$ are the received signals for receivers $i$ and $j$ ). This estimate is placed in the array DTOA using INDEX as a guide. This subroutine can also generate a plot of the above integral depending on the Hag PLTCC.

CROSINFO (N. ARRAY, J1, J2, SHIFT, DTA, FWHM, TC)

This subroutine exanines $N$ elements in the array ARRAY for a maximmm. The time corresponding to this point is used in estimating the delta time of arrival which is returned in DTA. J1 and J2 are the two receiver indices and SHIFT is the amount of time displacenent found in the input array. 


\section{ETTYDEF}

This subroutine is a r.ammy routine for the proper functioning of the OINK library.

\section{ETTYNOP}

This subroutine is a dummy routine for the proper functioning of the OINK library.

FF' (NT, NF, DT. TVAL, CFT. FVAL, WORK)

This subroutine computes the forward Fourier transform of a function. NT values of the function in the time domain are taken from TVAL (where D'T is the time increment between successive values) and converted to NF values in the frequency domain. The resulting complex spectrum is output in CFT while the magnitude of these values are output in FVAL. WORK is used as a scratch array.

\section{FILTERS}

This subroutine establishes the necessary information to define the receivers. The time domain response's are stored in TVAL. The complex Fourier transforms are stored in FIL. The magnitudes of the spectral values are stored in FVAL. Time delays to be associated with each filter are stored in RC'VRDLY.

FNTOA (VAL1. VAL2, NPTS, TINE, TOA, LEFT, RIGHT)

This subroutine estimates the time of arrival for a given receiver by the frequency-modulation method initially developed by R. Massey (gromp SST-7, LANL). It uses the cnvelope of the received signal in VAL1 to define a peak region. The bonndaries of this region are returned in LEFT and RIGHT. The actual received signal in VAL2 is examined over this region. A function which is the square of the difference between sucessive zeros of the signal versus the time values in TIME is constructed in VAL2 (overwriting the previos values). A straight line is then fit to this function. The point where this line crosses the time axis is returned in TOA as the time of arrival for that receiver.

\section{FRANGE (IESC)}

This subroutine determines the frequency range to be used in generating time domain plots so that the plotted signal will fit within the time window allotted. IESC is the escape code entered by the userer.

FREQPLOT (YVAR, IST. ND, NCURVES. NPLOTS, IOP'T)

This subroutine generates plots in the frequency domain. YVAR is the array of values to be plotted. IST is the starting index for each colum of the array while ND is the sumber of values to plot for

each of the NPLOTS. NCURVES is the number of curves to overplot on each graph. IOPT indicates the type of plot resired. 
FRFIL (FLO, FHI, COEF, FLTR)

This subroutine constructs a wide-band filter by smoothly patching together exponential functions in stch a way that there is $50 \%$ transmission at the specified low and high frequencies of FLO and FHI. COEFF governs the steepness of the exponentials. The resulting complex function is returned in FLTR.

\section{GETNOISE}

This subroutine retrieves noise data from a file whose name is given in NSFILE and then sorts the values.

GETNP (FR, DM, DS)

This subroutine calculates the noise parameters DM and DS corresponding to a given frequency FR by interpolating in the random noise table.

\section{GETRCVR (NR)}

This subroutine retrieves tabular data from a file whose name appears in SRFILE and which defines the frecuency response of a given receiver. NR is the index of the receiver.

\section{GET'TASK (I'TASK, FLAG1, FLAG2, FLAG3)}

This subroutine requests from the user the task that is to be performed (returned in ITASK) and calls the appropriate menu rontines to establish the necessary parameter values. FLAG1, FLAG2, and FLAG3 indicate whether paraneters were modified by the nenus corresponding to Transionospheric: Propagation, Satellite Detection, and Signal Processing respectively.

\section{GETTEXT (CL.UE, IERR)}

This subroutine retrieves textual information from the file TIPC.TXT, using CLUE as the string in angle brackets that sets off the particular message and returning in IERR an error condition code.

\section{INITMENU}

This block data subprogram initializes the various menu options.

\section{INITTF}

This subroutine initializes the array of times in TIME and frequencies in FREQ. It uses the time increment. JT and the number of samples points NPTS to do this. It also defines TMAX, TMID), and FMAX. 
INITVAL

This subroutine performs the necessary initialization when TIPC is started up.

INITVEC (ARRAY, LOW, HIGH, NUM, LOGSCALE)

This subroutine establishes an array of NUM values in ARRAY by interpolating between the low and high values (LOW and HIGH respectively). LOGSCALE indicates whether interpolation is to be done on a log scale or not.

IOFIRS (CIN)

This function in the OINk library determines the position of the first significant character in the string C.IN.

IOGETC (CPROMP, CESCAP, CINOUT, IESC)

This subroutine in the OINK library presents the prompt in CPROMP to the user and accepts the user's input of a string in CINOUT. CESCAP contains a string of "escape" characters. The status of the user's input is returned in IESC.

IOGETI (NEXPEC, CPROMP, CESCAP, IINOUT, NFOUNI, IESC)

This subroutine in the OINK library presents the prompt in CPROMP to the user and accepts the user's input of an integer value or array in IINOU'T. NEXPEC is the number of values to be expected. CESCAP contains a string of "escape" characters. The number of values entered is returned in NFOUND. The status of the user's input is returned in IESC.

IOGETR (NEXPEC, CPROMP, CESCAP, RINOUT, NFOUND, IESC)

This subroutine in the OINK library presents the prompt in CPROMP to the user and accepts the user's input of a real value or array in RINOUT. NEXPEC is the number of values to be expected. CESCAP contains a string of "escape" characters. The mumber of values entered is returned in NFOUND. The status of the user's input is returned in IESC.

IOGFIL (CPROMP. CESCAP, CSTAT, CFNAME, IESC)

This subroutine in the OINK library presents the prompt in CPROMP to the user and accepts the user's input of a file nane in CFNAME. The string in CSTAT is then used to determine the file's status. CESCAP contains a string of "escape" characters. The status of the user's input is returned in IESC. 


\section{IOINIT (CSYS, CASE, NWIDTH)}

This subroutine in the OINK library initializes the internal variables that are system dependent or global in nature. This must be called before any other OINK subroutine is invoked. CSYS contains the operating system keyword ("vms," "ctss," or "unix"). CASE indicates the case in which character information is returned to tle program from some of the OINK routines ("u" or "l"). NWIDTH defines the width of the termincal sireen or window in characters.

\section{IOLAST (CIN)}

This function in the OINK library determines the position of the last significant character in the string CIN.

\section{IONMEN (NITEM, CTITLE, NEXPEC, CPROMP, IOPT, NFOUND)}

This subroutine in the OINK library handles menus with integer-labeled options. NITEM is the number of items to appear on the menu. CTITLE contains the title to appear above the menu (may be a mull string). NEXPEC indicated the number of options that the user is expected to enter. The array of pronpts for the menu items is in CPROMP. The user's choices are returned in IOPT. The number of menu items chosen is returned in NFOUND.

\section{IOOFIL (LUNIT, CPROMP, CESCAP, CSTAT, CFNAME, IESC)}

'This subrontine in the OINK library prompts the user with CPROMP and accepts the user's input of a filc name in CENAME. The string in CSTAT is used as the status in attempting to open the file using the logical unit number specifice in LUNIT. CESCAP contains a string of "escape" characters. The status of the user's reply is returned in IESC.

\section{IOUSER}

This is a dummy routine for the proper functioning of the OINK library.

IOYORN (CPROMP, CESCAP, CYORN, IESC)

This subroutine in the OINK library presents the prompt in CPROMP to the user in the form of a yes or no question and accepts the user's response in CYORN. CESCAP is a string of "escape" characters. The status of the user's reply is returned in IESC.

IPCPB1

This subrontine generates a super-(Ganssian input signal.

\section{IPCPB2}

This subroutine generates a modified Canusian input signal. 
IPDELTA

This subrontine generates a set of delta functions for an input signal.

IPENP

This subrontine generates double exponential function for an input signal.

IPROP

This subroutine simulates the effect of the ionosphere on the input signal. It first adds the noise recpested to the input signal. Then it applies to the Fourier transform of the input signal a transfer function that includes phase shifts as a function of frequency in order to simulate the deterministic: aspects of the ionosphere. I: icintillations are requested, a stochastic transfer function is generated th is also applied to the signal. This subroutine can generate a plot of the transionospheric signal if indicated by PLTTIS.

\section{IPTABLE}

This subroutine generates an input signal from tabular data. The name of the file which contains the data is found in IPFILE.

\section{LE'TOA (ARRAY. NPTS, TIME, TOA, LEFT, RIGHT)}

This subroutine estimates the time of arrival for a given receiver by the Leading Edge method. The envelope of the NPTS values of the received signal is in the array ARRAY. Two points on the leading edge where the signal drops to $2 / 3$ and $1 / 3$ of the peak value are found. A straight line is drawn between these two points. and the point where the line intersects the time axis is declared to be the time of arrival for that receiver. The time values in TIME are used for this purpose. The time of arrival is returned in TOA. Also returned are boundaries of the signal peak region, LEFT and RIGHT, for plotting purposes.

MENUDT (FLAG, IESC)

This subroutine requests from the nser all the information needed to perform a delta-time-of-arrival study. FLAG indicates whether any of the parameter values wore changed. IESC is the escape code corresponding to the user's last response.

MENUDU (FLAG, IESC)

This subroutine requests from the user all the information needed to perform a delta time of arrival uncertainty study. FLAG indicates whether any of the paraneter values were changed. IESC is the escape code corresponding to the user's last response. 


\section{MENUPS (FLAG, IESC)}

This subroutine requests from the user all the information needed to perform sigral processing. FLAG indicates whether any of the parameter values were changed. IESC is the escape code corresponding to the user's last response.

\section{MENUSD (FLAG, IESC)}

This subroutine requests from the user all the information needed to perform signal detection. FLAG indicates whether any of the parameter values were changed. IESC is the escape ode corresponding to the user's last response.

\section{MENUTP (FLAG, IESC)}

This subroutine requests from the user all the information needed to perform transionospheric propagation. FLAG indicates whether any of the parameter values were changed. IESC is the escape code corresponding to the user's last response.

\section{NEWSEED (SEED)}

This subroutine uses the current system time to establish an initial value for SEED which is used in the generation of random numbers.

\section{NOISE}

This subroutine defines the noise that is to be added to the input signal. It can also generate plots of the tabular noise, the input signal plus noise, or the spectrum of the input signal plus noise.

\section{NOISE1.}

This subrontine generates white noise for the input signal.

\section{NOISE2}

This subroutine generates frequency-dependent, noise for the input signal based on the tabulated rata in a file. The name of the file is stored in NSFILE.

NOPRNT

This is a dumny routine for the proper functioning of the OINK library.

PARANS' (NODE)

'This subroutine either waves the paraneter values in a file or restores those values from a file depending on the string in MODE. The user is prompted for the name of the parameter file. 
PARSEMSG (MSG, KEYWORD, VALUE, IESC)

This subroutinesextracts the keyword and value from the tirst segment of an intormation message in MSG which is included with tabular data. These are retured in KEYWORD and VALUE respectively. The messages minus the first segunent are returned in MSG. An error condition is returned in IESC:

PAIISE

This subroutine prints ont an innocuons message and waits for any response from the user.

\section{PLOTEXIT (TTY)}

This subroutine in NCAR.FOR torminates any graphics output.

\section{PLO'TINIT (TTY)}

This subroutine in NCAR.FOR prepares for graphics output.

PLOTXY $(X, Y$, ISTART, NDIM, NPTS, NC', IPLT, NPLT, IOP'T, XLABEL, YLABEL, TITLE1. 'TITILE2, MESSAGE, 'T'TY)

This subrontine in NCAR.FOR produces one or more $X-Y$ plots. The columns of $Y$ are plotted as a function of $X$ starting at ISTART. NDIM is the column dinension of the two-dimensional array $Y$. NPTS is the number of points to plot. NC is the number of curves to plot per graph. IPlit is the index of the plot (from 1 to 4 ) that indicates where in the page it is to be plotted in case there is more than one plot. NPLT is the total number of plots. IOPT indicates the type of scales to use (1 for linear $X$, linear $Y ; 2$ for linear $X$, logarithmic $Y ; 3$ for logarithmic $X$, linear $Y$; and 4 for logarithmic $X$, logarithmic $Y$ ). XLABEL contains the label for the $X$ axis while YIABEL contains the label for the $Y$ axis. TITLE1 and TITLE2 contain titles and subtitles to be (lisplayed at the top) of the page. MESSAGE contains secondary information to be displayed inmediately below the titles. TTY indicates the type of terminal being used.

POLY $(N, C O E F, X)$

This function returns the value of a polynomial at $X$ which is specified by $N$ coefficients stored in COEF.

PULSE (IESC)

This subroutine recpuests from the user the information needed to determine the input signal. Returned in IESC is the escape code corresponding the user's last resiponse. 
Q(TAD)

This subroutine performs the quadrature filtering algorithm. It sums the received signal itself and the received signal shifted out of phase by 90 degrees with a fietitions local oscillator. It can plot these cuadrature results if indicated by PLTQUAD.

RANDG (MEAN, SIGMA, SEED)

'This subrontine generates Ganssian random values with mean and standiard deviation specified by MEAN and SIGNA respectively. SEED is automatically upelated.

RANDU (SEED)

This subroutine generates miform random values between \%ero and one. SEED is antomatically updated.

RESTORE (NANE, VALUE, INDX)

This subrontine restores the value of a parameter specified in a line of the paraneter file. The parameter's name is given in NAME. If it is an array, the index is given in INDX. The character-string containing the value is given in VALUE.

SA'TDE'T'

This subroutine models the detection of a transionospheric signal by a specified receiver. It can also generate plots of the received signals.

SAVEC (LUN, NAME, VARIABLE)

This subroutine encodes the value of a character-string variable stored in VARIABLE along with its name in NAME and writes this ont to the parancter file using the logical unit munber specified in LUN.

SAVEI (LUN, NANE, VARIABLE)

This subroutine (nocodes the value of an integer variable stored in VARIABLE along with its name in NAME and writes this ont to the parameter file using the logical unit number specified in LUN.

SAVEL (LUN, NAME, VARIABLE)

This subroutine encodes the value of a logical variable stored in VARIABLE along with its name in NAME and writes this ont to the parameter file using the logical unit number specitied in LUN. 
SAVER (LUN, NAME, VARIABLE)

This subroutine encoles the value of a real variable stored in VARIABLE along with its name in NAME and writes this ont to the paraneter file nsing the logical mit number specified in LUN.

SE'T'TEC (VALUE)

This subroutine establishes a new value for TEC and other global variables whose values depend (on TEC.

SIGNAL

This subrontine constructs the input signal and its Fourier transform. It can also generate plots of the input signal.

$S O R T(N, X, Y$, NDIM, N( $)$

This subroutine sorts the $N$ elements of array $X$ in ascending order and makes sure the elements in each column of $Y$ are permuted in exactly the same way. NDIM is the column size of $Y$ while NC is the rumber of columms to permute.

SQRLAW

This subroutine passes the signal power through a low-pass filter to determine its envelope.

\section{TASKDT}

This subroutine performs a Delta Time of Arrival study by simulating the reception of a transiomospheric signal by specified receivers for varions values of TEC and estimating the differences in times of arrival.

\section{TASKKDU}

'This subroutine generates varions realizations of white noise or scintillations and then simmlates the reecetion of the resulting transionospheric signal by specified receivers. The nean and sigma of the differences in arrival times are calculated.

\section{TASKPS' (INDEX)}

This subroutine applies one of a mumber of signal-processing algorithms to the transionospheric signal received by the satellite receivers. INDEX is a pointer into the array DTOA. 


\section{TASKiSD}

This subrontine determines what signals are received by ach of the receivers whese frepuency response has been specified by the user.

\section{TASKTP}

This subroutine determines what happens to the input signal as it passes through the ionosphere.

TIMEPLOT (OFFSET, YVAR. IST, NID. NCURVES. NPLO'TS. IOP'T)

This subrentine generates one or more plots in the time domain of the values in the array' YVAR such that the values in TIME are offset by an anount in OFFSET. IST is the starting index of the values to be used. while ND is the number of values to be used in the plots. NCURVES is the mumber of curves to plot on each graph. NPLO'T's is the total mumber of plots. and IOP'T is an indicator of the type of plot to make.

\section{TSHIFT (CFT, TS. VAL, SAVE)}

This subroutine multiplies the complex spectrum in (F) by an appropriate function so that the signal in the time domain is shifted by an anome TS. The resulting time domain representation is returned in VAL. SAVE indicates whether the spectrum is to be uprated or not.

WINDOW (ARRAY.N, CUTOFF, IST. ND)

This subroutine searches the elerents in ARRAY forward from I and hackwarl from $N$ for the first value that exceeds CUTOFF times the largest element in absolute value. The left index is returaed in IST while the total number of points in between the limits is returned in NJ).

YSCALE $(Y, N$. SCALE, LABEL)

This subroutine in NCAR.FOR scales $N$ values in the array $Y$ and returns the scaling factor in SCALE. It also returns a scaling label in LABEL that can be used in labeling the ordinates.

\section{SAMPLE RUNS}

To illustrate the capabilities of 'TIPC' and familiarize the user with the format of its output, we present the results of representative runs for each of the five tasks discussed in section II. These rums are limited in scope and are meant only to illustrate varions aspects of the code. At the same time. however. the selected cases do tonch on some interesting lacets of propagation. signal detection. and signal processing. 


\section{A. Transionospheric Propagation}

Two sample input pulses, the delta function and the super-Ganssian function, were run through the transionospheric propagation task for four values of the frequency coherene bandwidth $\left(f_{\text {con }}=\infty\right.$, 20. 10. and $1 \mathrm{MHZ}$ ) and two signal-to-noise ratios $(S \mathrm{SR}=100$ and 10.000$)$. 'The defante pulse parameters were selected in each case and the results are summarized in Figures $3 a \mathrm{a}$ and da j, respectively. Figure 3 a is a plot of the input delta function while Figure 36 is a plot of its speetrum. Note that the signal is zero exeept at $t=4 \mu$ s. where it has amplitude $1 / \Delta t$ (the triangular shape is an artifact of the pletting routine and the finite sampling) and its spectrum is Hat from zero to the Nycuist frecunency with amplitude mity.

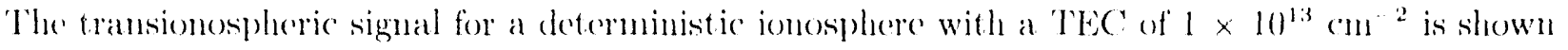
in Figure 3e. Though not apparent in this plot. the initial inponlse is separated into its individual freguency components with the high frecueneies arriving first. 'The amplitude of the hight-frepuency components is larger because the delta function has a flat spectrum and the dispersion due to the iomesphere is higher at lower frecunencies. The sudden rise in signal amplitude at $14 \mu$ is an artifact introduced by aliasing.

The effect of seattering on the transionospheric signal is depicted in Figures $3 \mathrm{~d}$ f. We see that the signal has been modulated in time over a scale defined by the temporal dispersion corresponding to the coherence handwidth. Thus, the temporal structure in the signal increases as Fon decreases and the modulation scale in time increases as a function of time. The latter result stems from our forcing the coherence bandwidth to be constant over the entire frecpuency range displayed. In general. a constant colerence bandwidth is not an areurate description of scattering because Foh varies as $f_{0}^{1}$. A gencral theoretical treatment of this problem is not yot available.

The inpact of Ganssian-distributed white noise on the signal is secen in Figures $3 g$ j. The input signal with a SNR equal to 100 is shown in Figure $3 \mathrm{~g}$. The corresponding transionospherie signal is ploterel in Figure 3h. Becanse of the tremendous dispersion introduced by the ionosphere, the amplitude of an initial impulse is greatly reduced relative to the noise. which represents a continuous signal that suffers ne reduction in anplitude due to dispersion. This explains the obvious reduction in signal-tonoise seen in Figure 3h. The results for a SNR of 10,00) are shown in Figures 3i j. For this case the shape of the transionospherie signal is barely discernible in the noise.

A similar set of results. Figures ta j. were obtained for the super-Canssian default pulse. This pulse shape. shown in Figure ad, has the corresponding spectrum shown in Figure ab. The latter speceral shape introduces a modulation in the transionospheric signal. plot ted in Figure te. relative to what one would ohtain for a flat spectrum (see Figure 30). Adelitional modulation is introduced in the

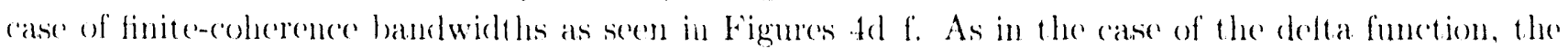
transionesplentic signal is lost in the noise for a SNR of 100 (Figure the and barely discernible for a SNli of $10 .(100)$ (Figure $4 \mathrm{j})$.

\section{B. Signal Filtering}

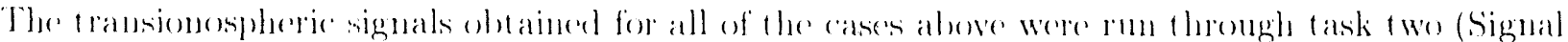

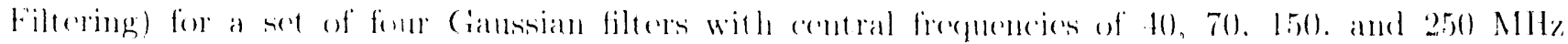
and a single bandwidth of $2 \mathrm{MHz}$ FllHA. The filter profiles are shown in Figures ba d while the

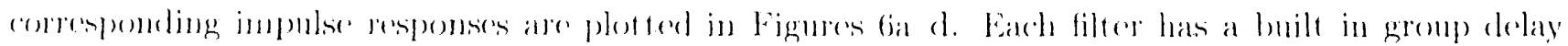

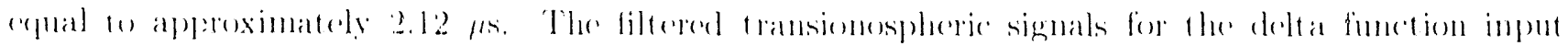


pulse are shown in Figures 7 a d. Because the bandwidth of these filters and the ionospheric TEC are sufficiently small, the dispersion introduced by the ionosphere across the filter is not readily observable in these signals. We do, however, observe a frequency-dependent delay in the arrival time of the peak signal. For example, the $40 \mathrm{MHz}$ signal (Figure $7 \mathrm{a}$ ) show's a total delay egual to the sum of the signal delay of $4 \mu$ s measured relative to a chesen absolute zere time, the filter delay of $2.12 \mu$, and the ionospheric delay of $7.97 \mu$ s explal to that expected for the central frecpuency of that filter. A similar result is obtained for the other filters. Wo also find that the total delay decreases with increasing frepuency in a manner consistent with the expected ionospheric delays. By tine-tagging the arrival of the signal in different receivers, it is therefore possible to determine the ionospherie TEC: Algorithms developed for this purpose have already been discussed.

The effect of finite coherence bandwidths on the filtered andonsonosperic signal is shown in Figures $8 \mathrm{a}$ d, 9a d. and loa d. When the coherence bandwidth of the ionosphere is larger than the filter bandwilth. the signal appears virtually maffected. Closere examination. however. reveals additional time chays and larger temporal widths that are consistent with the expected mean delays and the time delay jitter for the given colerence bandwidth. When Fron falls below the bandwidth of the filter, significant distortion of the signal is ohereved (Figures 10a d). Additional delays and a broader filtered signal are also seen. Each of these results represents a single realization of a statistical set of potential onteones. It is apparent that the statistical delays obtained in the presence of strong scattering introduce random uncertainties in the computed ionospherie TECs. The additional temporal structure introduced at low coherence bandwidths exacerbates the problem by making it diflecult to determine an arerurate time of arrival.

The filtered transionesphere signats for a delta function and a SNR of 100 are shown in Figures 11 a 1. It is apparent that the signal is washere out by the combination of ionospherie dispersion and noise for

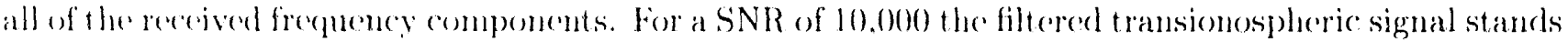

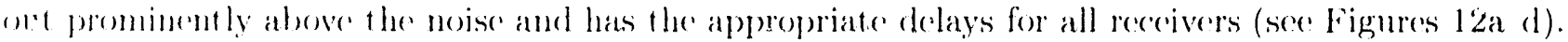

A romplementary set of results for the super-(ianssian pulse is displayed in Figures 13a d, 1 ta d,

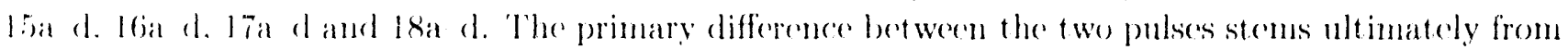
their Fourier spertra. For example. the results show that for a SNR of 100 , it is possible in the case of

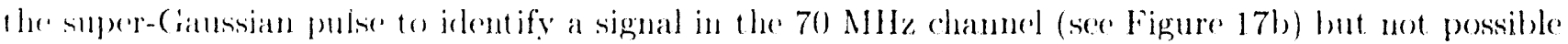
in the ase of the delta function. As seen in Figure the the super-Craussian pulse has a peak in its neertrum at 70 MHz. The amplitude of this peak is greater than that at the other channel frecuenciess and is sulfieienty large to be detected above the noise. For the super-Gaussian pulse and a SNR of

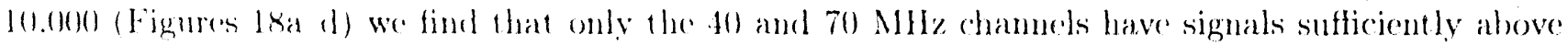
the moise to be measured. Whereas signals in all chamels are detectable for the delta function (see Fignres $12 a$ 1). These results indieate that for narrowband systems. signal dentection relies strongly on

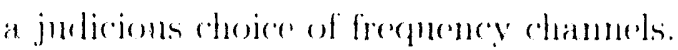

The chsontial aspects of signal desection that are relecant to identification of important spectral features in the radiated pulse are smmmarizal in a series of plots that show the receried power in the

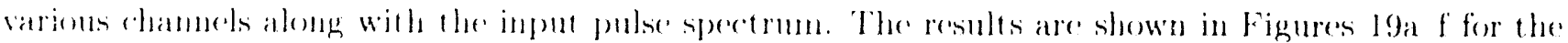

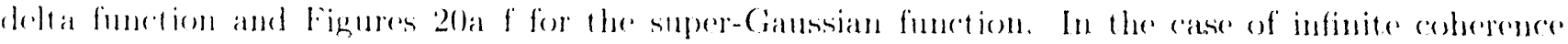

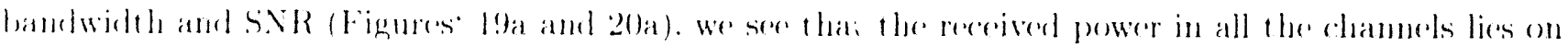

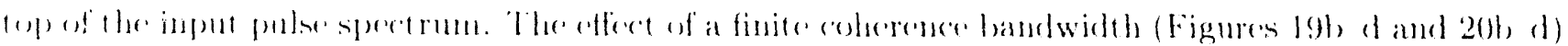

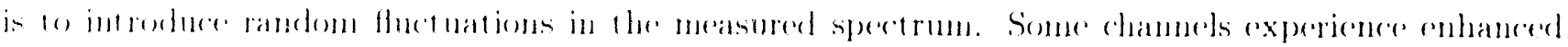

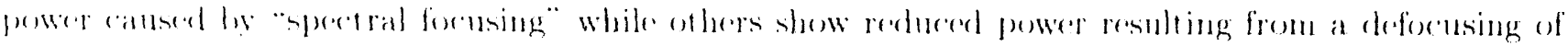


the signal in that channd. The effect of noise (Figures $190 \mathrm{f}$ and $20 \mathrm{f}$ ) is to obscure the signal in some channels relative to others.

These results illustrate the difficulties encountered in measuring signal characteristipe in the presence of ionospheric dispersion, scattering, and Gaussian white noise. The magnitude of these offerts depends strongly on the value of TEC, $F_{c o h}$. and SNR, and it is important to have a good estimate or measurement (preferably in situ) for these paraneters. It is apparent that narrowband systoms suffer from the additional problem of not knowing the optimm channel frecuencies a priort for arbitrary pulse shapes. Broadband systems that cover the entire VHF mage from 30 to $300 \mathrm{MHz}$ are far superior for the task of signal reconstruction.

\section{Signal Processing}

The defanlt delta-function along with a wideband filter characterized by a central frecuency of 130 $\mathrm{MHz}$ and a FWHM of $40 \mathrm{MHz}$ were used as input to the quadrature and square-law detection subtasks. The absolute magnitude of the filter transfer function is shown in Figure 21 while the results for quadrature detection and square-law detection are shown in Figures $22 \mathrm{a}$ d and 23, respectively. 'The' I and $Q$ components and the phase of the received signal show the effect of the local oscillator (chosen to be $130 \mathrm{MHz}$ ). As the signal chirps through the $130 \mathrm{MHz}$ filter frecuency, the relative beating of the signal and the local oscillator first decreases and then increases with time, as is evident, in Figures 22 a, b, and d. The minor differences between the transionospheric signal obtained with scluare-law detection and the amplitude (Figure 22c) obtained with quadrature detection are associated with the fact that the default 10 $\mathrm{MHz}$ low-pass filter was used for square-law detection while the receiver filter has a 40) $\mathrm{MHz}$ FWHM.

Results for the TOA algorithms were generated for the default dolta function and two filters, namely, a wideband filter from $100400 \mathrm{MHz}$ and a narrowband filter with a central frecueney of 70 $\mathrm{MHz}$ and a baidwidth of $6 \mathrm{MHz}$. The filter transfer functions are shown in Figures $24 a$ and $\mathrm{b}$. Throe cases, corresponding to (1) infinite coherence bandwidth and 70 dlB SNR, (2) infinite coherence bandwidth and 30 dB SNR, and (3) 10 MHz coherence bandwidth and 70 dB SNR, were run for cach TOA algorithm and receiver. The transionospheric signal for ach case is shown in Figures $25 a$ a The results using the FNTOA technique are shown in Figures 26a c and 27 a c for the wideband and narrowhand filters, respectively. The TOA obtained for any given filter is measured relative to the zero time in me initial time window and therefore corresponds to a signal delay phus some delay canted by the filter tharacteristics. In the case of the narrowband filter, the FNTOA technicpue arrives at a TOA of $4.469 \mu$ s, which equals the signal delay phus a nominal filter delay of 0.469 ps (soce Figure 26 tia). This result would in general he independent of 'TES provided the FM'TOA technicue compensates aceurately

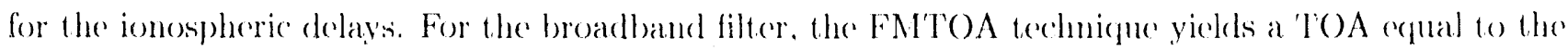
signal delay to an accuracy of one nanosecond (see Figure 27a). The offect of a SNR of 1,000 is shown

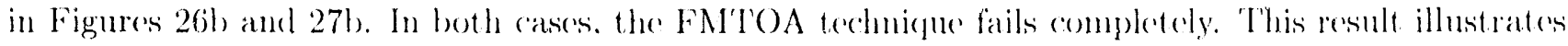
the strong effect of dispersion on the measured SNR at the receiver and our ability to measure the 'TOA with this technicgue. For a coherence bandwidth of $10 \mathrm{MH}$ we find that the narrowband channel makes

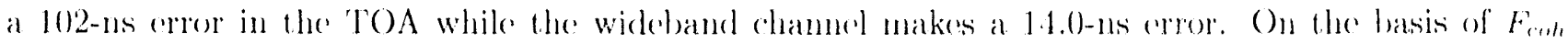
we expere the mo jitter in the arrival time to be approximatedy 16 ns. For this particular realization, we find that the narrowhand channel shows a larger crorer than expected while the wideband chamel performs as expected. As a result of the phase mixing that oceurs within the wideband chanuel and finite sampling. the FNTOA terhnique has difticulty measuring the oscillation period at any instant in 
time (as evidenced by the large relative variation in period compared to the narrowband chamel) but nevertheles: measures the chirp rate more accurately because of the broader bandwidth.

Results for the leading-edge analysis are shown in Figures $28 \mathrm{a}$ c and $29 \mathrm{a}$ c. Two basic conclusions can be drawn from this exercise. First, we find that the broadband measurements yield a greater SNR and faster rise times on the leading edge than the narrowband measurements. As a result, the widehand TOA determinations are more consistent and accurate for all cases. Second, because Gaussian white noise introduces uniform phase nixing, techniques that rely on phase information (e.g.. zero-crossing measurements in the FMTOA algorithm) are at a disadvantage relative to wideband amplitude measurements, provided a reasonable SNR is achieved. Indeed, the leading-edge technique performs better wh h the wideband receiver than the FNTOA algorithm in these examples.

The ontput of the cross-correlation analysis includes both a DTOA and a TEC estimate that is derived from equation (16). The results are shown in Figures 30a c. This technique yields DTOAs that are accurate to within $15 \mathrm{~ns}$ in the presence of both noise and scattering and thus performs bet ter than either the FNTOA or leading-edge algorithms for the cases considered. The TEC estimate is approxinately $30 \%$ higher than the actnal input value; however, the accuracy can be improved significantly by constructing a look-up table for TEC as a function of DTOA.

\section{DTOA Study}

The clefanlt delta function and two Gaussian filters with central frequencies of $150 \mathrm{MHz}$ and 5) $\mathrm{MHz}$ and bandwidthe of $6 \mathrm{MHz}$ were used as input to the DTOA study. The leading-edge, timetagging algorithm was invoked to obtain TOAs for cach channel over a range of TECs from 0.1 to $10 \times 10^{13} \mathrm{~cm}^{2}$. The results, shown in Figure 31, illustrate some of the limitations of TIPC. We ser that the plot is characterized by two straight lines of different slope with the break occurring at a TEC of approximately $4.5 \times 10^{13} \mathrm{~cm}^{-2}$. Because the DTOA is proportional to TEC, ideally one expects to obtain a single straight line. The discrepancy results from the fact that the dispersion across the 5) MHz filter becomes comparable to the $10-\mu$ s window and significant aliasing occurs for TECs $25 \times 10^{13}\left(\mathrm{Cu}^{-2}\right.$. This result leads to crroneous values for the TOA of the $50 \mathrm{MHz}$ channel. The user (an by ans this problem by selecting a larger sampling time, therehy increasing the size of the window. The tradeoff is that the maximum frequency (Nycuist frequency) that can be treated is reduced. The user should take care to select an appropriate range of TECs.

This task will generate, for any specified set of receivers, a look-11) table that allows the user to determine a TEC for any given measurenent of DTOA. This analysis represents the first step in removing ionospheric effects from the received signal.

\section{DTOA Uncertainty Study}

The defanlt delta function and the two Ganssian filters specified for the DTOA study were used as input to the DTOA uncertainty stuly. The TEC was fixed at $1 \times 10^{13} \mathrm{~cm}^{-2}$ and Gaussian white noise wat arked to the input signal. The resulting filtered transionospheric signal was time-tagged with the leveling-edge algorithm for signal-to-noise ratios ranging from $10^{2}$ to $10^{5}$ (see Exps. (7), (8), and (9) for implementation of noise). A mean DT' $) A$ and the standard deviation from the mean were calculated using 25) realizations of each SNR The results are shown in Figures 32a and b. Note the rapid reduction in performance betow a SNR of $10^{4}$ (see Figure 32b). A similar study was undertaken for colnerence bandwidthe ranging from 1 to $20 \mathrm{MHz}$. The results are shown in Figures 33a and b. The magnitude 
of the DTOA uncertainties over the range cotsidered are smaller by an order of magnitude than those obtained in the case of noive, suggesting that scattering of the ENIP by ionospherice irregularitics is less disruptive. Note the rapid deterioration in performane below a colecene bandwidth of $10 \mathrm{MH} \%$. As Froh approaches the filter bandwidth, amplitude variations are introduced to the leading-eage of the received signal, resulting in additional uncertaintios in time-tagging.

\section{FUTURE ENHANCEMEÑTS}

TIPC represents a powerful tool for exploring important aspects of transionospheric propagation, the detection of RF signals following propagation and signal analysis of pulse waveforns. The code was designed to be versatile enough both to address general questions regarding the effects of ionospheric propagation on received signals and to simmlate actual receiver systems and their measurements of transionospherice signals. As such, TIPC is both an edncational tool and an applications code. Future enhanements to 'TIPC will be in the area of input, ontput. (the output is primarily in the form of graphics at present) and in the development of a reconstrection algorithm.

The output of future versions of 'TIPC will inchule texe files with relevant results from each of the tasks and subtasks selected by the user. A log file containing a listing of the input parancters and a history of the execution of varions tasks and associated results will be created. In aldition, the user will be given the option of writing ont the transionespheric signal-filtered transionospheric signals, results from quadrature detection and square-law detection, and tables of DTOA vs. TEC (obtained from the DTOA Study task) for specified filter pairs. The nser will also be given the option of specifying a transionospheric signal or a set of filtered transionospheric signals in tabular form. In this way the signal-processing algorithms developed for TIPC can be applied to measurements and, for example, a TEC can be estimated with the DTOA algorithms for the specified data. Fimally, a signal Reconstruction task will be created. This task will have two options: Inverse Filter and Correlation Analysis. The first option will create an inverse filter for the ionosphere based on a TEC estimate obtained from the Signal Processing task, create the inverse filters for the specified set of receivers. and apply these filters to the specified filtered transionospheric signal to obtain an estimate of the intial signal. The second option will perform a cross-correlation between a set of calculated or sperified filtered transionosplerie signals and a corresponding set (read in from a specified text file) obtained for a known input signai. Depending on the amplitude of the cross-correlation, the user can determine whether or not the known input signal is a viable candidate for the specified measurements.

\section{REFERENCES}

Aarons, J. H., E. Whitney, and R. S. Allen, 1971, Proc. IEEE, 59, 159.

Bahar, E., and B. S. Argrawal, 1976, Radio Sci.. 11, No. 11, 885.

Barabanenkov. Tu. N., Yu. A. Kravtsov. S. M. Rytov. and V. I. Tanarskii. 1971, Sorn. Phys. Usjerkhi. 13. No. 5. 5510 .

Basn. S., S. Basu, and K. Kahn. 1976, Radio S'i.. 11. No. 10, 821.

Basu. S., and M. C. Kelley. 1979. Radio S'ci. 14. 171. 
Basu. S., S. Basin. J. P. Mullen, and A. Birshby, 1980, Geophys. Res. Lett.. 1, No, 4, 259.

Bast, S., E. Mackenzic, E.. S. Basu, H. O. Carlson, D. A. Hardy, R.. J. Rich, and R. (E. Livingston, 198\%: Rudio Sir.. 18. No. (i. 1151.

Bilitza, D.. 1986, "IRI: Recent Developments," Radio S'ri, 21, 343.

Bogusch. R. L.. F. W. Crugliano, D. L. Knepp, and A. H. Michelet, 1981, Pror. IEEE, 69, No. 7, 787.

Bowker. H. (i...J. A. Ratcliffe, and D). H. Sherin, 1950, Proce. Phys. Soc., Lomelon, 242, A856, 75.

Budden. K. G., 1965, J. Atmos. Terr. Phys., 27, 155.

('osta, E., and M. C. Kolley, 1976, Geophys. Res. Lett., 3, No, 11, 677.

Dana. R. A.. 1988. "ACIRF User's Gride," Volume 1: "Theory and Examples." DNA(0)1-87-(-0169, MlRC-R-1198, Mission Rescarch Corporation.

Daviess. K. 1965. Ionsphertic Radio Propagation, National Bureau Standards Monograph 80) (U.S. Govcrmment Printing Office, Washington, DC), 470 pp.

Dyson, P. R., I. P. McClure, and W. B. Hanson, 1974, J. Geophys. Res., 79, 1497.

Fejor. B. (i. and Mi. (․ Kelley, "Ionospheric Irregularitics," 1980, Ren. Geophys., 18, 401.

Fremonw. E. I., R. L. Londabrand, R. C. Livingston, M. D). Consins, C. L. Rino, B. (: Fir, and R. A. Lomg, 1978. Radio S'ri. 13. No. 9. 1, 167.

Fremonw, E. J.. R. ('. Livingston, and D. A. Miller, 1980, J. Atmos. Terr. Phys., 42, 717.

Johnson. A. 1981. Air Forer (ieophysies Laboratory fochnical Meno No, 56, V-1.

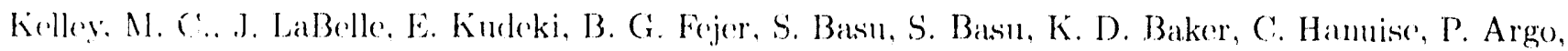
R. F. Woodman. W. E. Swarte and D. T. Fartey, 1985, preprint.

Kinepp. D. L., 1982. Defense Nuelear Agency report DNA-TR-81-78.

Linepp, D. L., and R. L. Bogusch, 1979. Mission Rescarch Corporation report DNA 5038'T, MRC-R-514. Livingston, R. (... 1980). Radio Siri, 15, No. 4. 8()1.

Lovedace, R. V. E., E, E. Salpeter, L. E. Sharp, and D. E. Harris, 1970, Ap, J., 159, 1047.

Martin, E... and J. Aarous, 1977. J. Geophys, Re's, 82. No. 19, 2713.

Pholps. A. R., and R. ('. Sagalyn, 1976, J. Crophys. Res., 81, No, 4, 515.

Rawer. K., D. Bilitza and S. Ramakrishmam, 1978a. Rew. of Cieophys. and Spa. Mhys., 16, No. 2, 177.

Rawer. K. and P. A. Bradley, 1987. editors of "International Reference Ionosphere Status 1986/87," Adramess in Sipa. Res.. 7. No. 6. 129 pages.

Rawer, K.. S. Ramakrishman, and D). Bilitza, 1978b, "IRI 1978," International Union of Radio Science (URSI). Brussels. Bolginum.

Rinu. ('. L... and J. Owert. 1980). Radio S'ri. 15, 479.

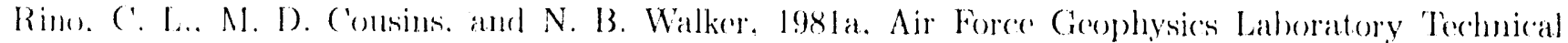
Menum No, 56i. IV-I.

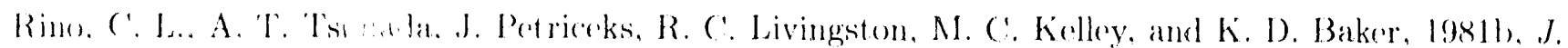
(iomplys. Res. 86. 2411. 
Rishbeth, H., and O. K. Garriott, 1969, Introduction to Ionosphlwy ir: Phylsics, in International (ieophysics Series, 14, J. Van Mieghern and Austin L. Hales, Eds. (Academic Press, New York, 1969)).

Rufenach, C. L., 1975a, Rudio Scri., 10, No. 2. 155.

Rufenach, C. L., 1975b, Radio S'ci, 10, No. 11, 973.

Salpeter, E. E., 1967, Ap. J.. 147, No. 2, 433.

Tatarskii, V. I., 1971. "The Effects of the Turbulent Atmosphere on Wave Propagation," U.S. Department of Commerce, National Technical Information Service, TT68-ij()464, 472.

Umeki, R., C. H. Liu, and K. C. Yoh, 1977, J. Gcophys. Res., 82, No. 19, 2752.

Wittwer, L. A., 1979, Defense Nuclear Agency report DNA-53(04D.

Wittwer, L. A., 1980, Defense Nuclear Agency report DNA-5662D.

Whitney, H. E., J. A. Klobuchar, J. Lloyd, and J. Austin, 1981, Air Force Geoplyysics Laboratory Technical Memo No. 56, III-I. 


\section{APPENDIX A. Flow Chart Tree}

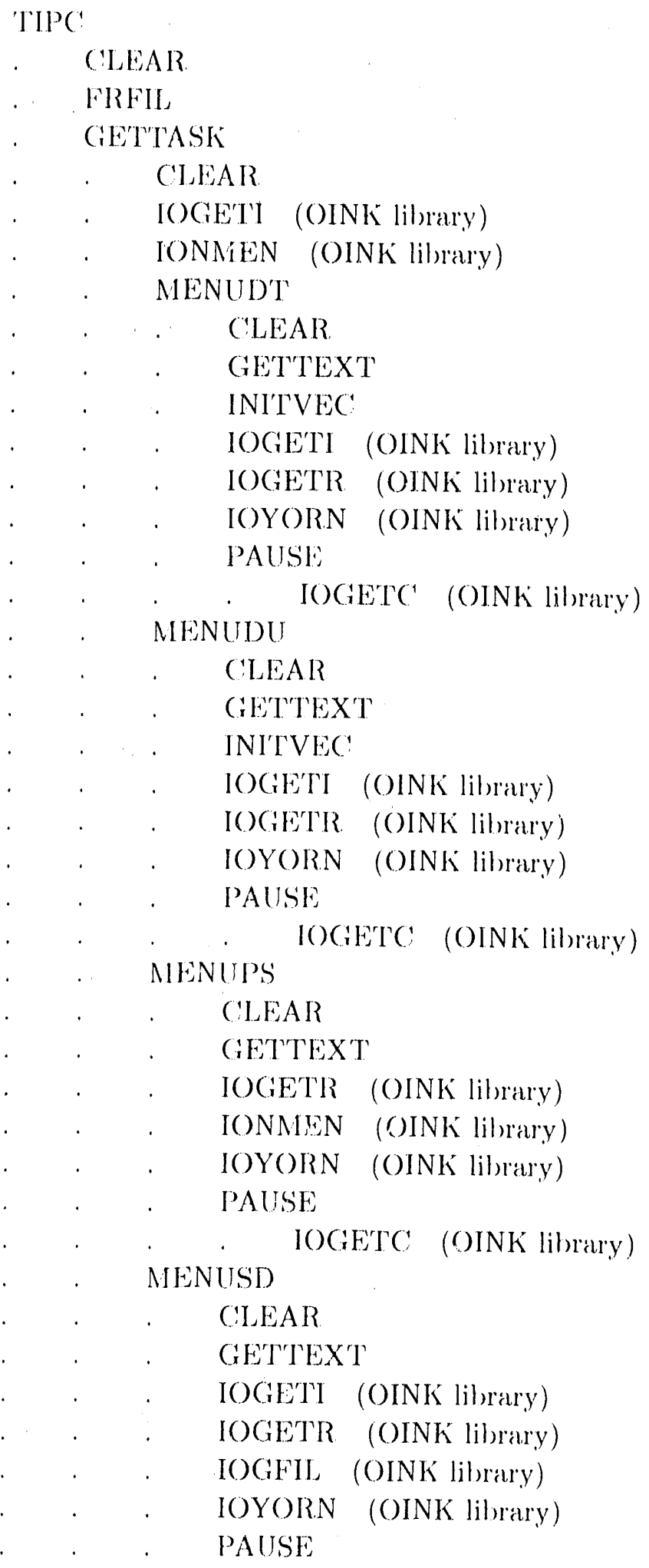




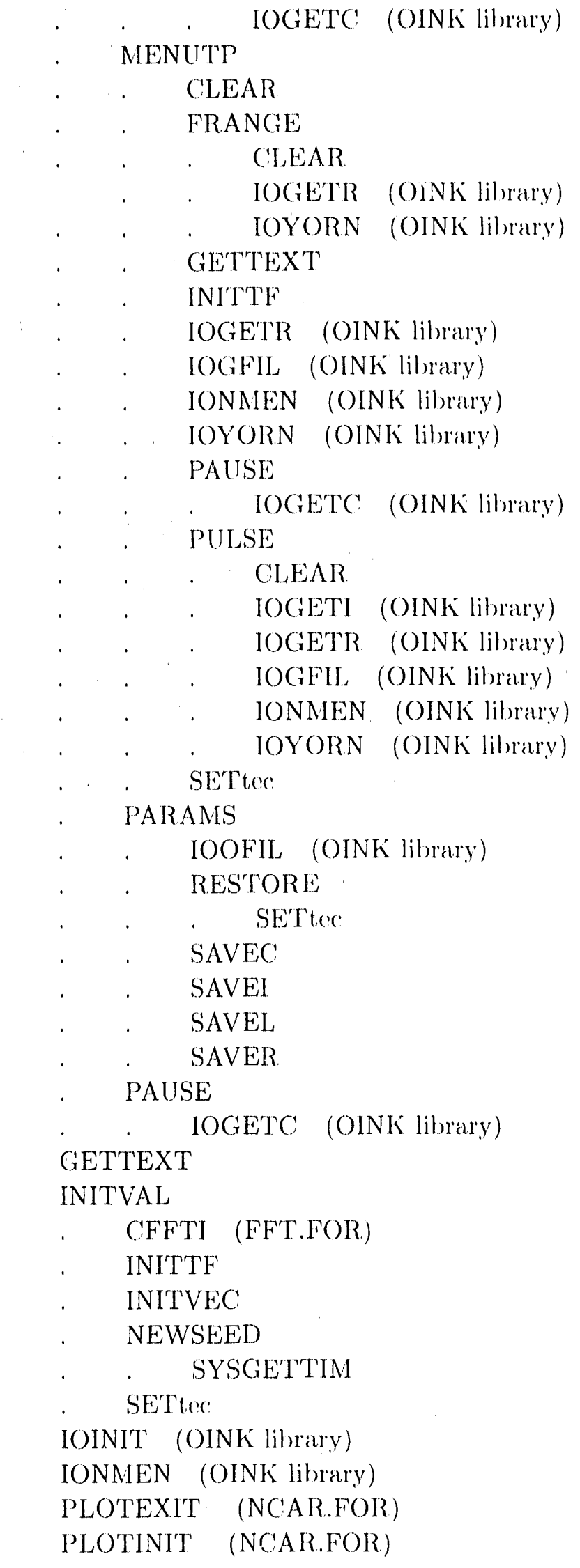




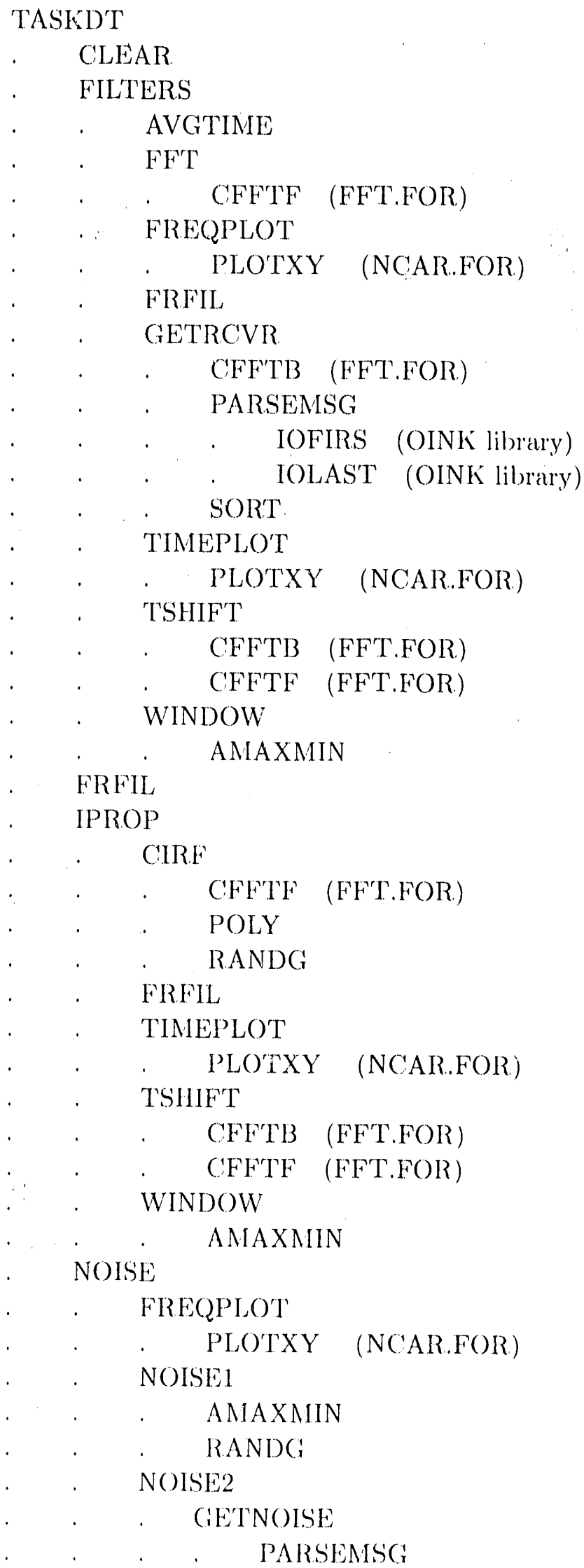




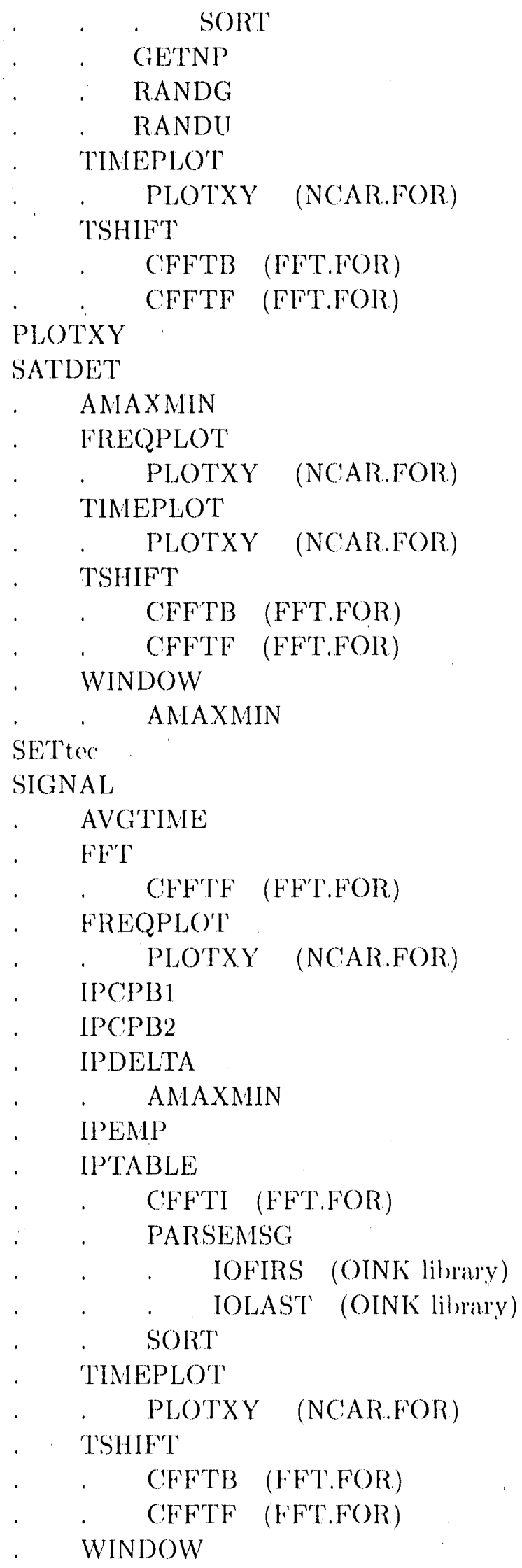




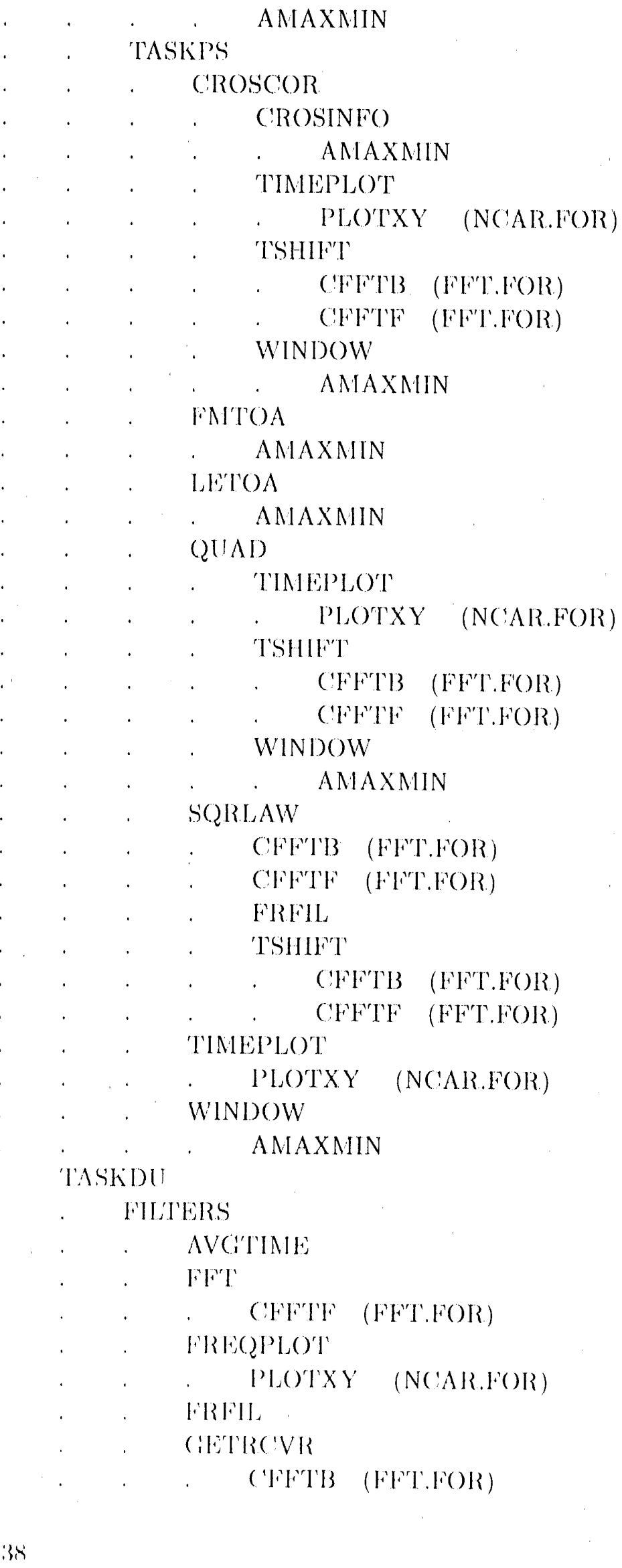




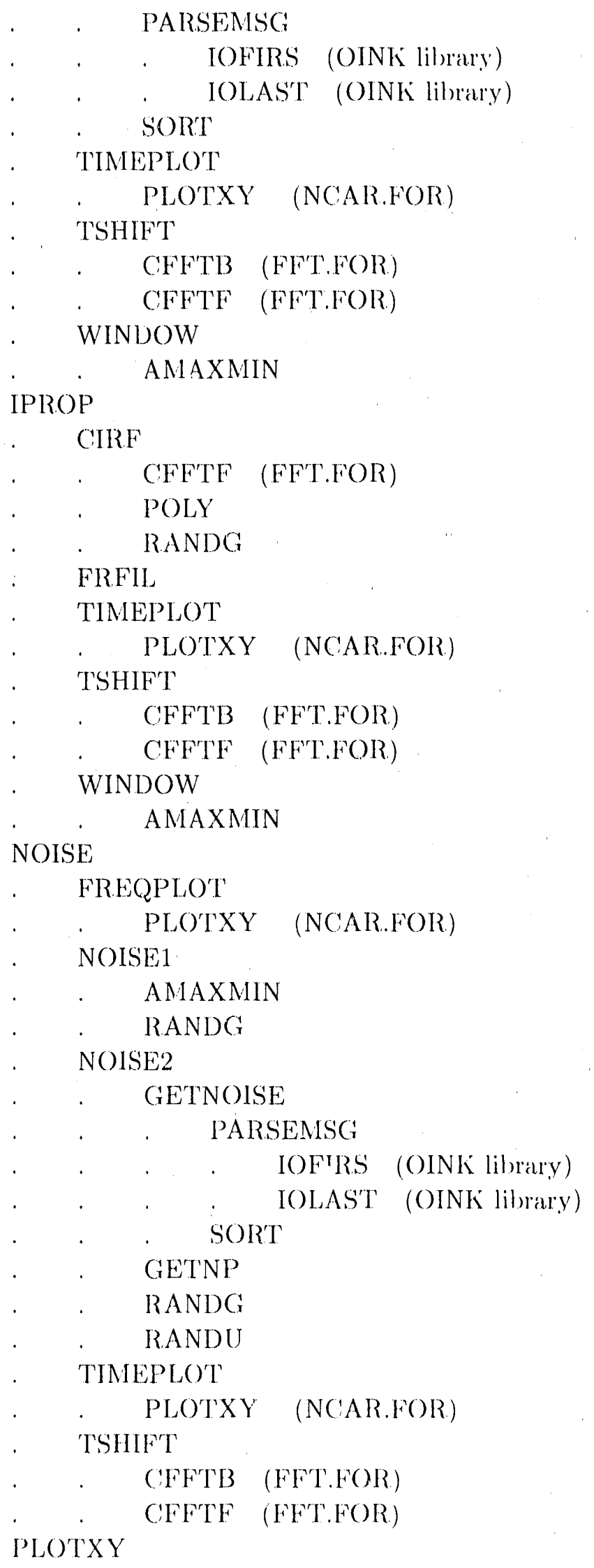




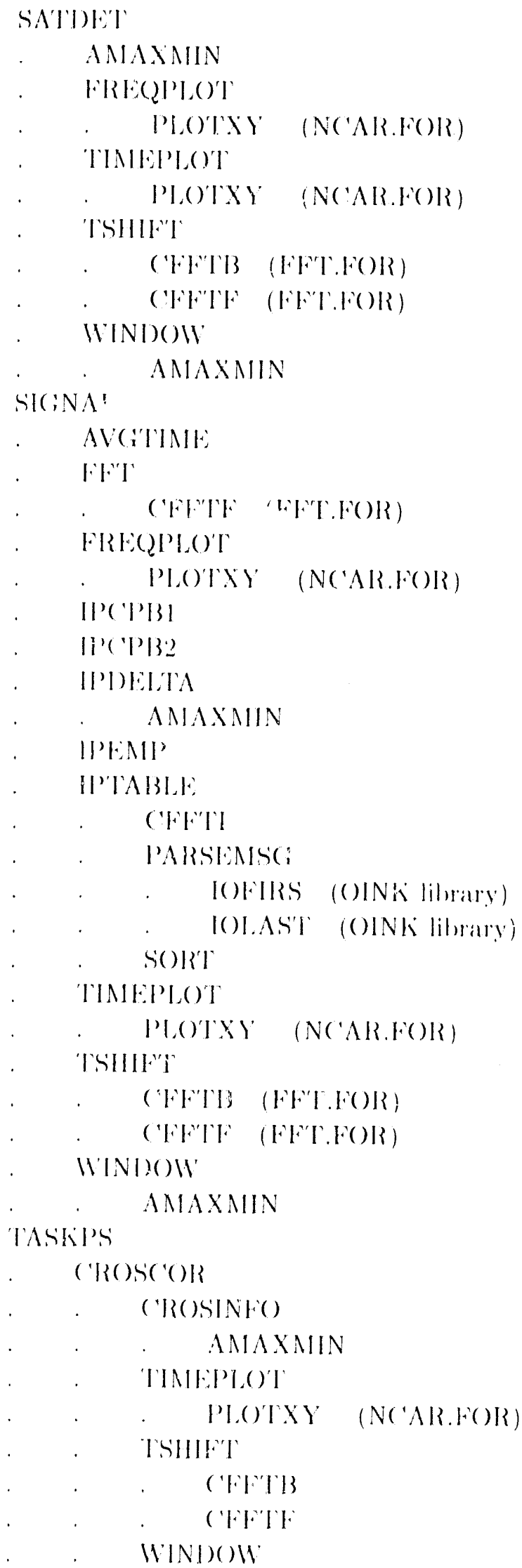




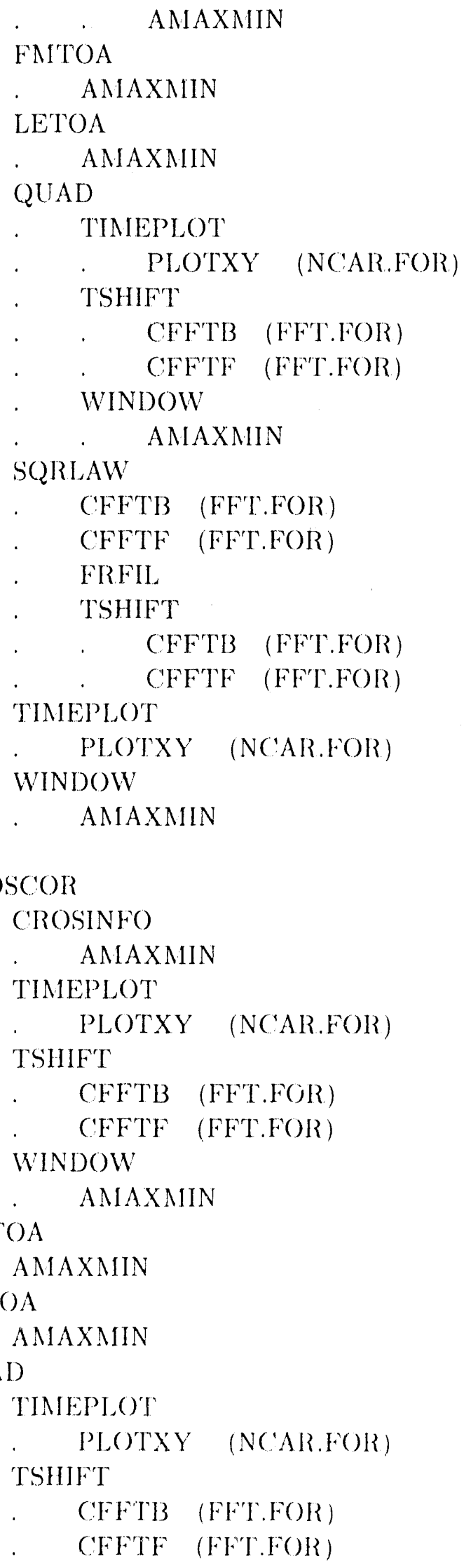




$$
\begin{aligned}
& \text { WINDOW } \\
& \text {. . AMAXMIN } \\
& \text { SQRLAW } \\
& \text { CFFTB (FFT.FOR) } \\
& \text { CFFTF (FFT.FOR) } \\
& \text { FRFIL } \\
& \text { SHIFT } \\
& \text { CFFTB (FFT.FOR) } \\
& \text { CFFTF (FFT.FOR) } \\
& \text { TINEPLOT } \\
& \text { PLOTXY (NCAR.FOR) } \\
& \text { WINDOW } \\
& \text { TASKSD } \\
& \text { AMAXMIN } \\
& \text { FILTERS } \\
& \text { AVGTINE } \\
& \text { FFT } \\
& \text { CFFTF (FFT.FOR) } \\
& \text { FREQPLOT } \\
& \text { PLOTXY (NCAR.FOR) } \\
& \text { FRFIL } \\
& \text { GETRCVR } \\
& \text { CFFTB (FFT.FOR) } \\
& \text { PARSEMSG } \\
& \text { IOFIRS (OINK library) } \\
& \text { IOLAST (OINK library) } \\
& \text { SORT } \\
& \text { TIMEPLOT } \\
& \text { PLOTXY (NCAR.FOR) } \\
& \text { TSHIFT } \\
& \text { CFFTB (FFT.FOR) } \\
& \text { CFFTF (FFT.FOR) } \\
& \text { WINDOW } \\
& \text { AMAXNIN } \\
& \text { FREQPLO'T } \\
& \text { PLOTXY (NCAR.FOR) } \\
& \text { SATDET } \\
& \text { AMIAXMIN } \\
& \text { FREQPLOT } \\
& \text { PLOTXY (NCAR.FOR) } \\
& \text { TIMEPLO'T } \\
& \text { PLOTXY (NCAR.FOR) } \\
& \text { TSHIFT } \\
& \text { (FFTB (FFT.FOR) }
\end{aligned}
$$




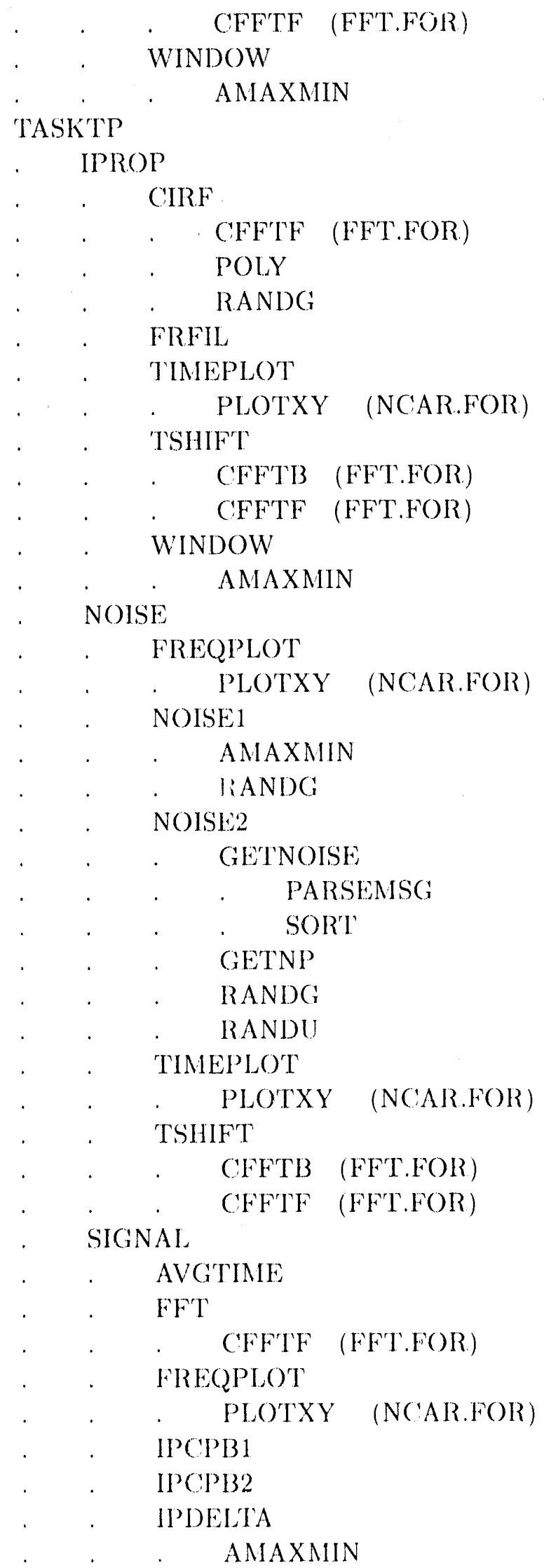


IPEMP

IPTABLE

CFFTI

PARSEMSG

IOFIRS (OINK library)

IOLAST (OINK library)

SORT

TIMEPLOT

- LOTXY (NCAR.FOR)

TSHIFT

CFFTB (FFT.FOR)

CFFTF (FFT.FOR)

WINDOW

AMAXMIN 


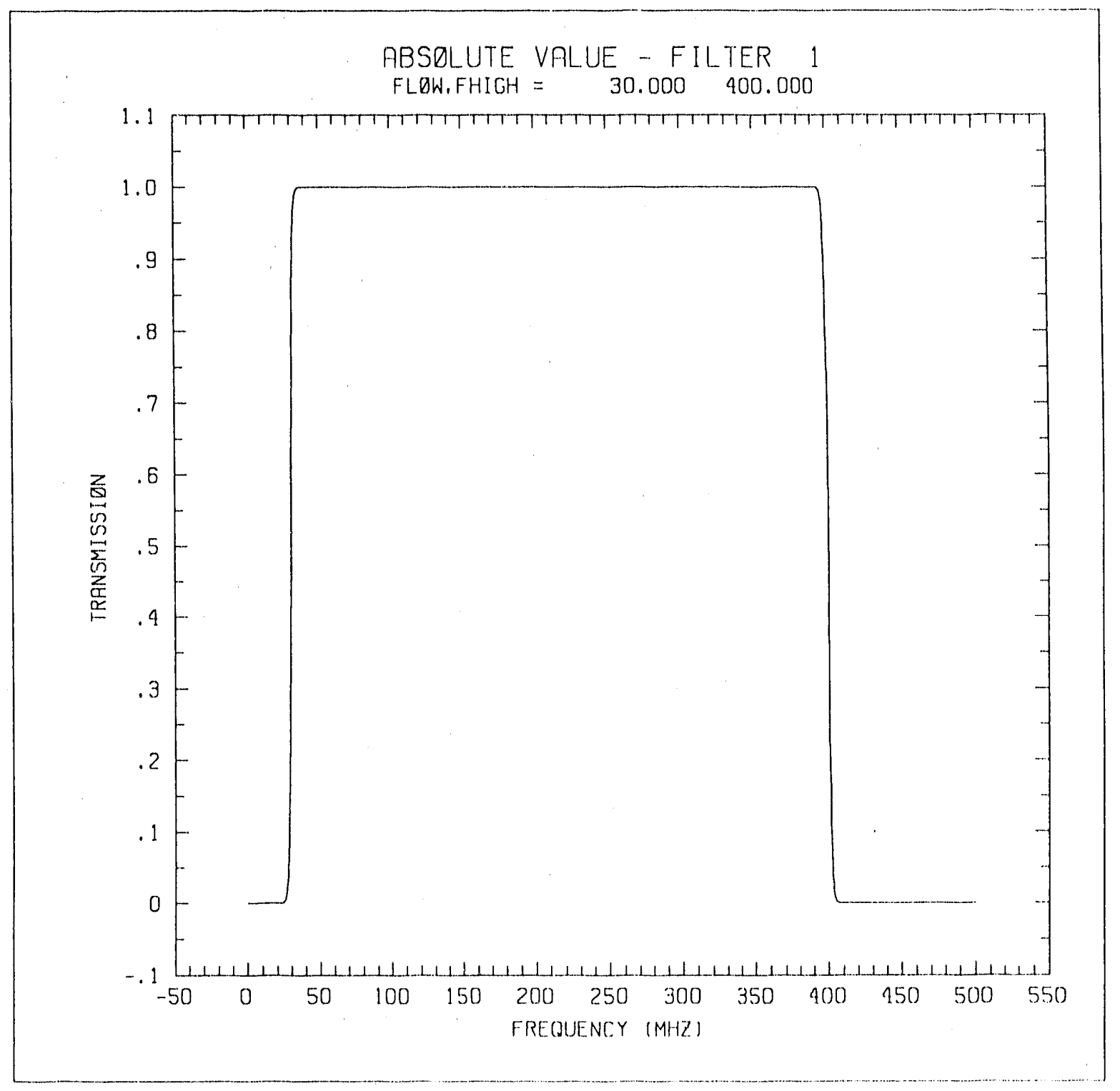

Figure 1 The filter shape used to limil the frequency range in the time domain to fit in the $10-\mu$ s window. This functional form preserves the signal amplitude through most of the lime domain and minimizes ringing near the edges of the window. 


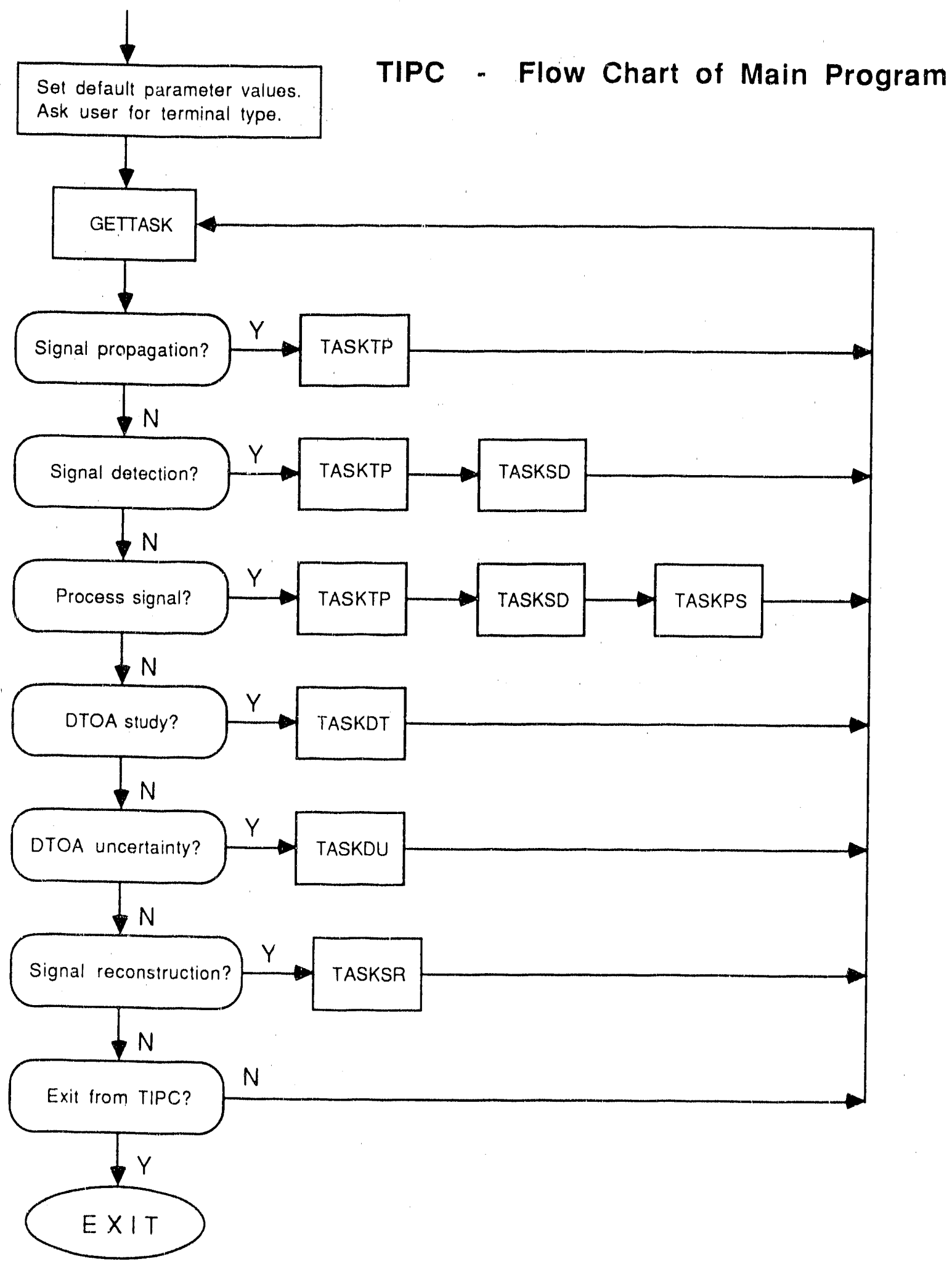

Figure 2 Flow diagram for TIPC. 


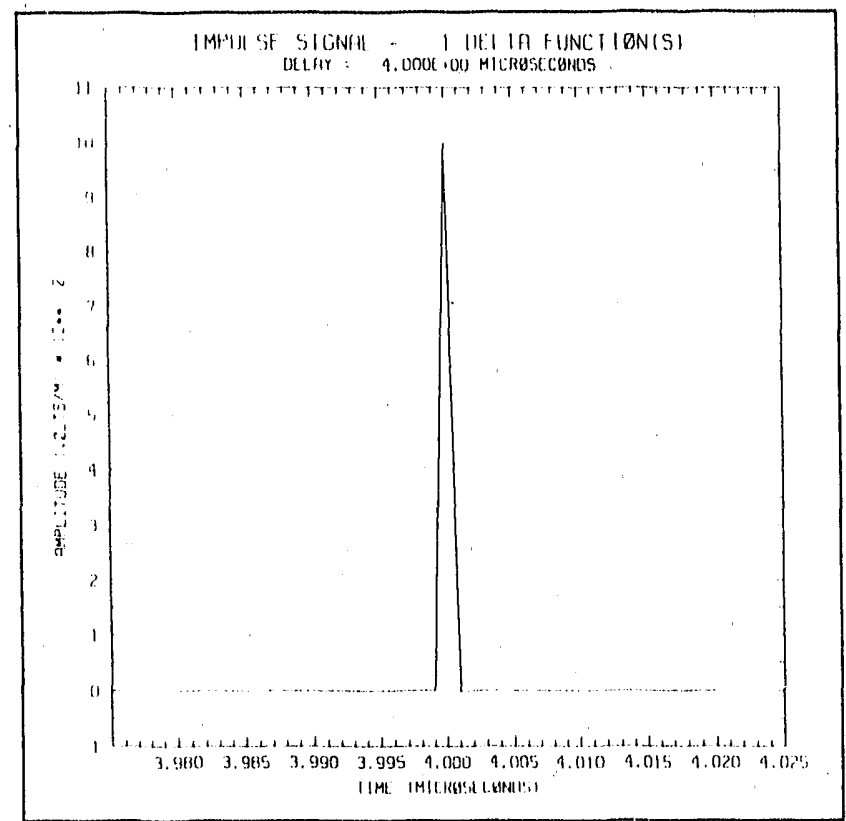

Figure 3a

Figure $3 b$

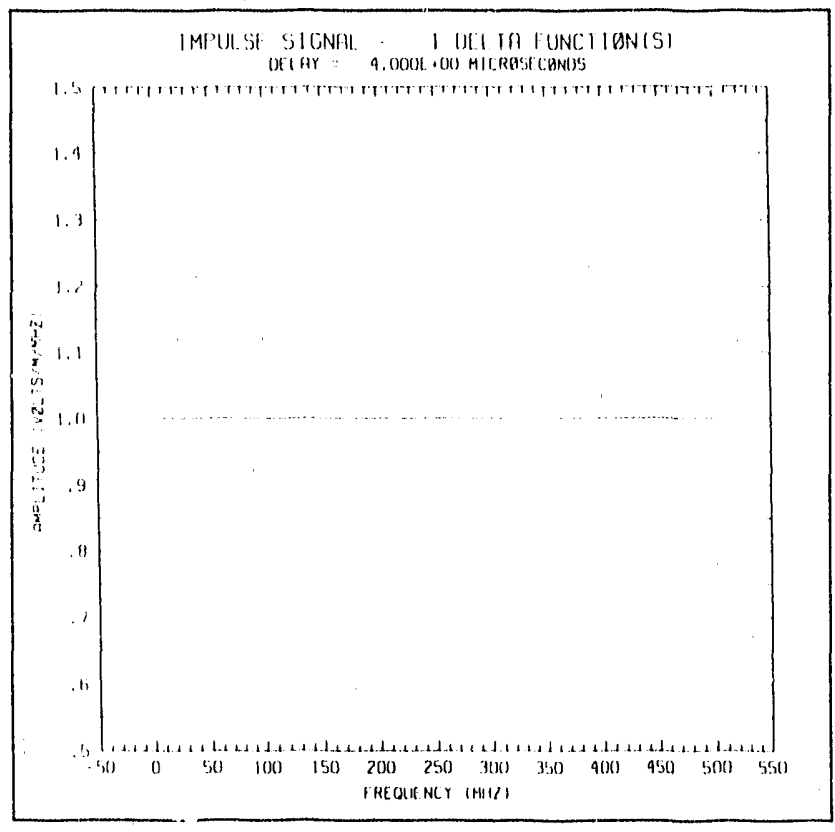

Figures 3a-j (a) Temporal plot of the default delta function. (b) FFT of the default delta function. (c)--(f) Corresponding transionospheric signals for a TEC of $1 \times 10^{13} \mathrm{~cm}^{-2}$, an SNR limited by numerical noise $(70 \mathrm{~dB})$, and $F_{\text {coh }}=\infty, 20,10$, and $1 \mathrm{MHz}$, respectively. $(\mathrm{g})-(\mathrm{h})$ Temporal plot of the noisy default delta function with an SNR $=100$ and the corresponding transionospheric signal for a TEC of $1 \times 10^{13} \mathrm{~cm}^{-2}, F_{c o h}=\infty$, and a SNR $=100$, respectively. (i)-(j) Temporal plot of the noisy default delta function with an SNR $=10,000$ and the corresponding transionospheric signal for a TEC of $1 \times 10^{13} \mathrm{~cm}^{-2}, F_{c o h}=x$, and an $S N R=10,000$, respectively. 


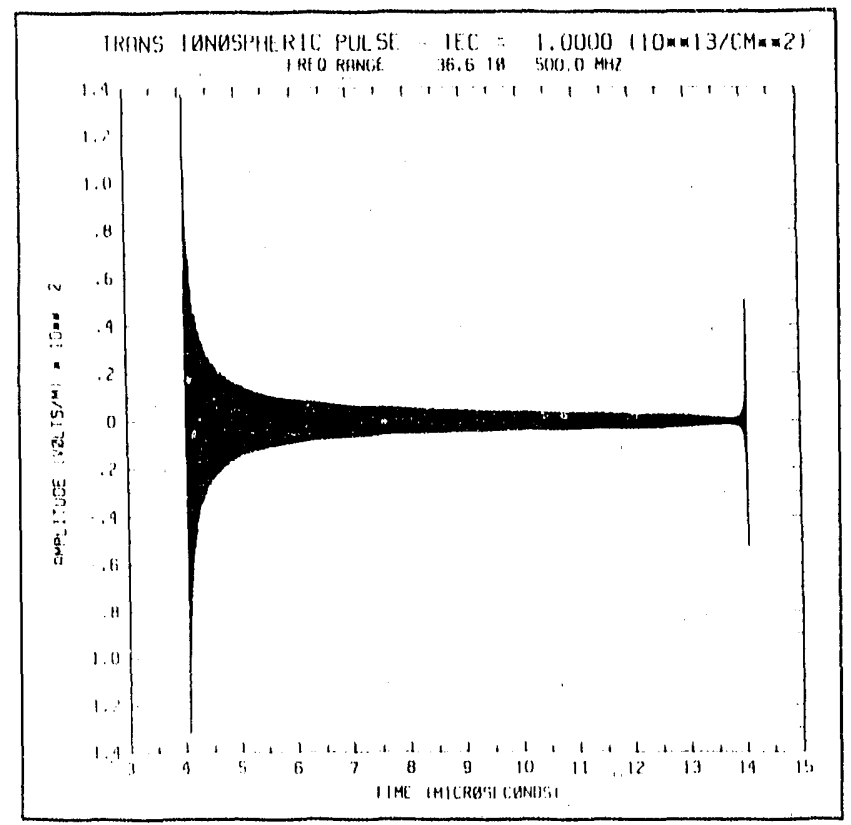

Figure 3c

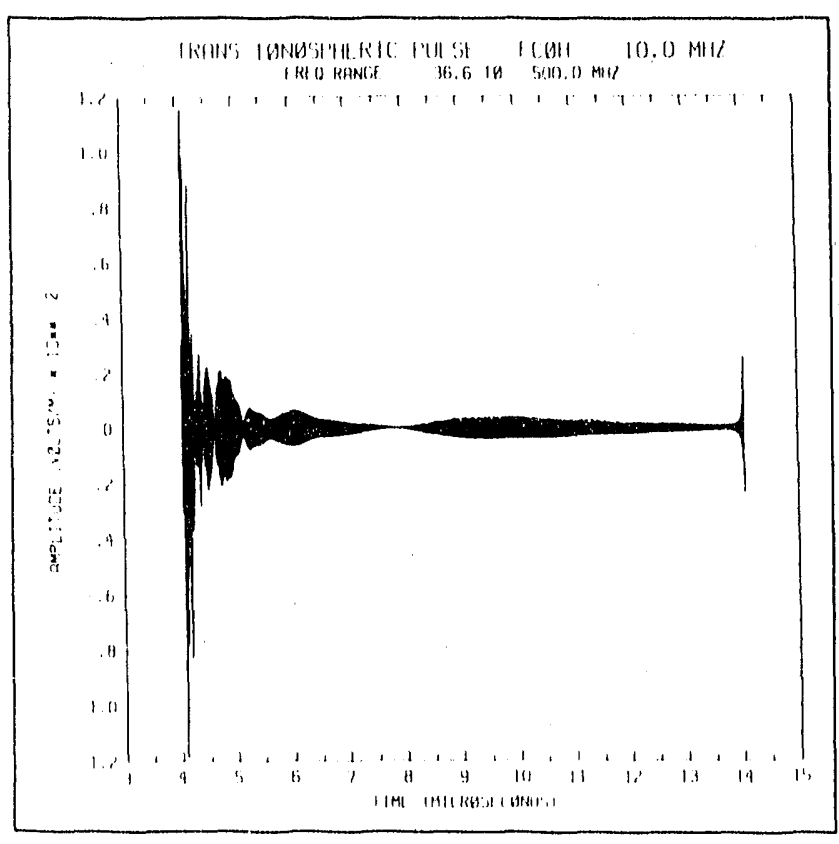

Figure 3e

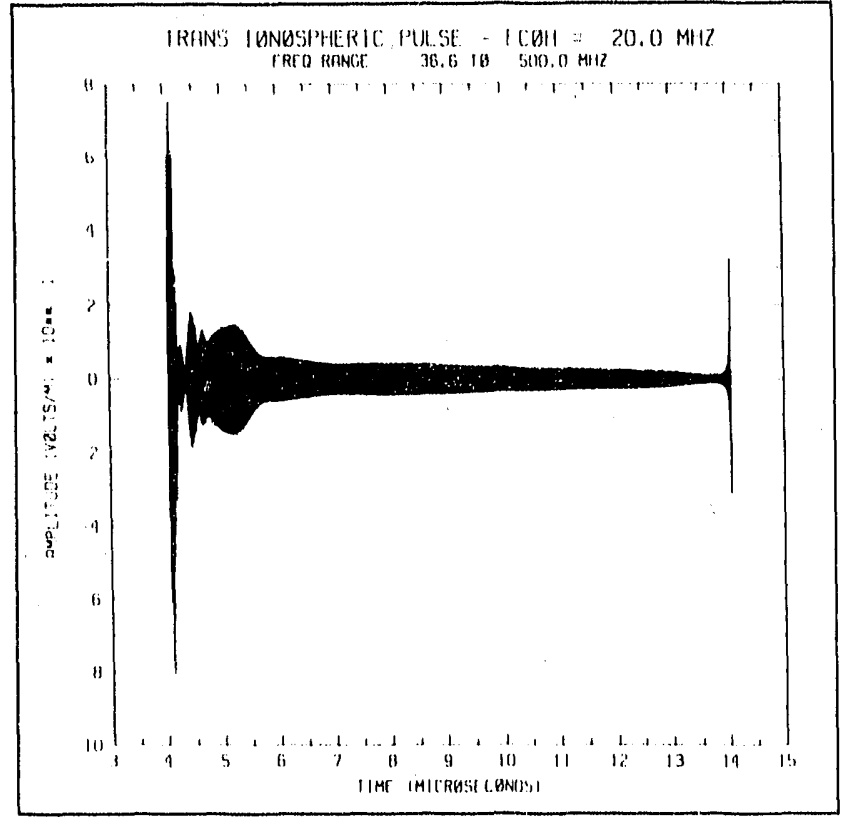

Figure 3a'

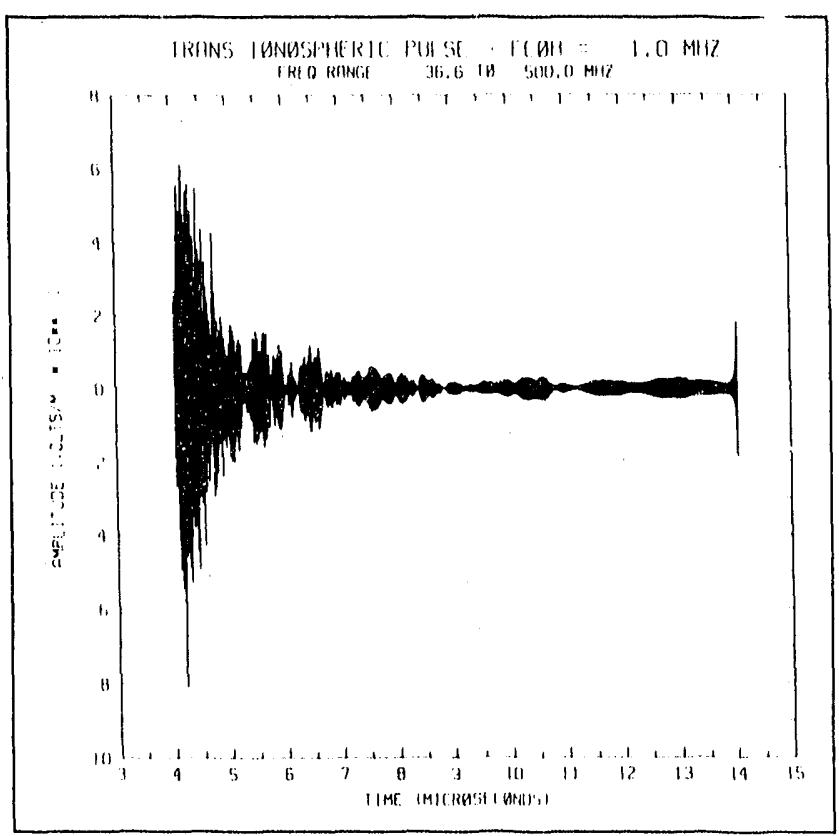

Figure 3f 


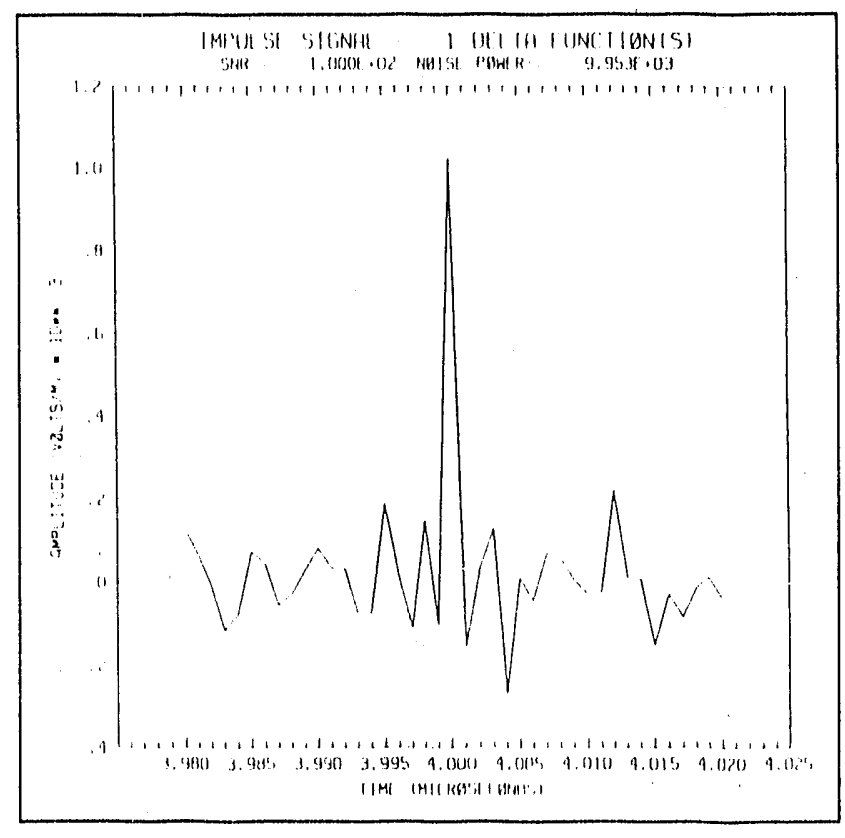

Figure 3g

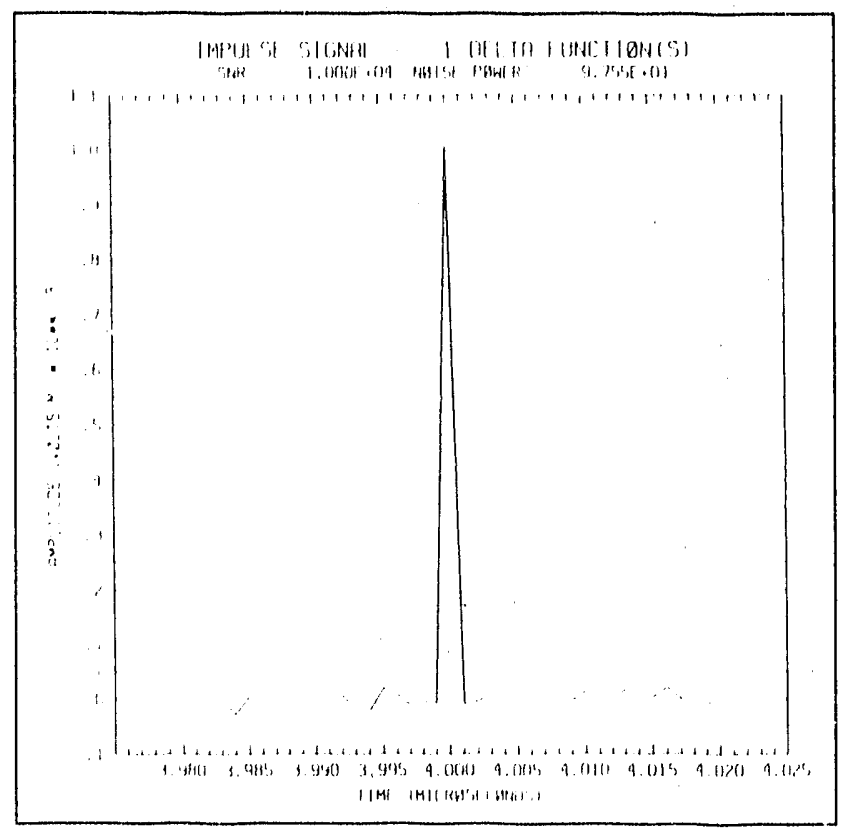

Figure 3i

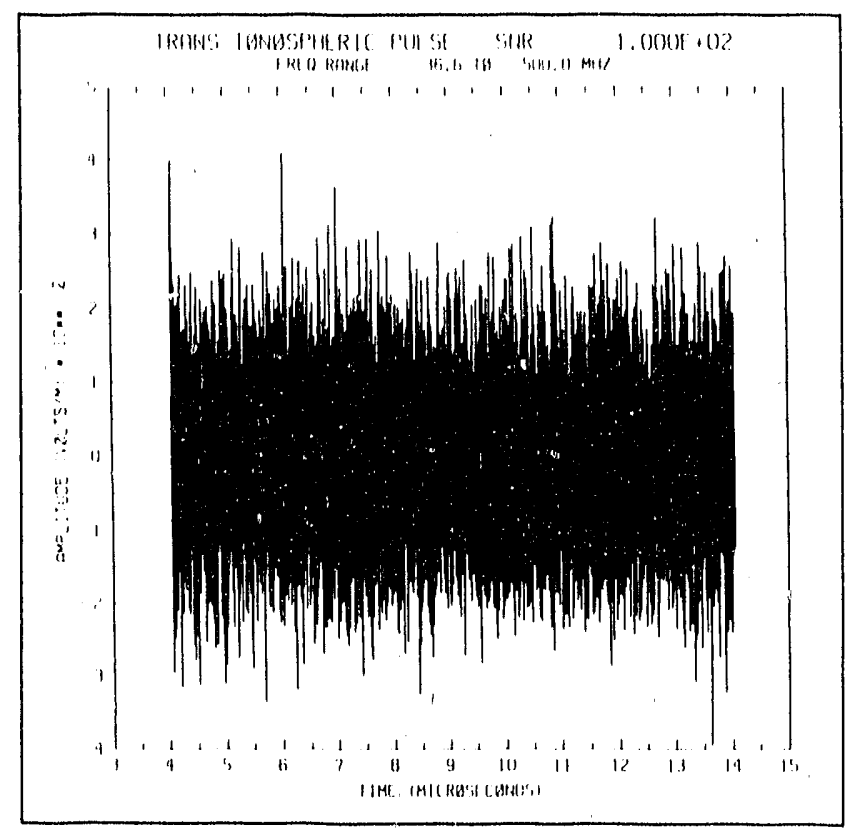

Figure 3 h

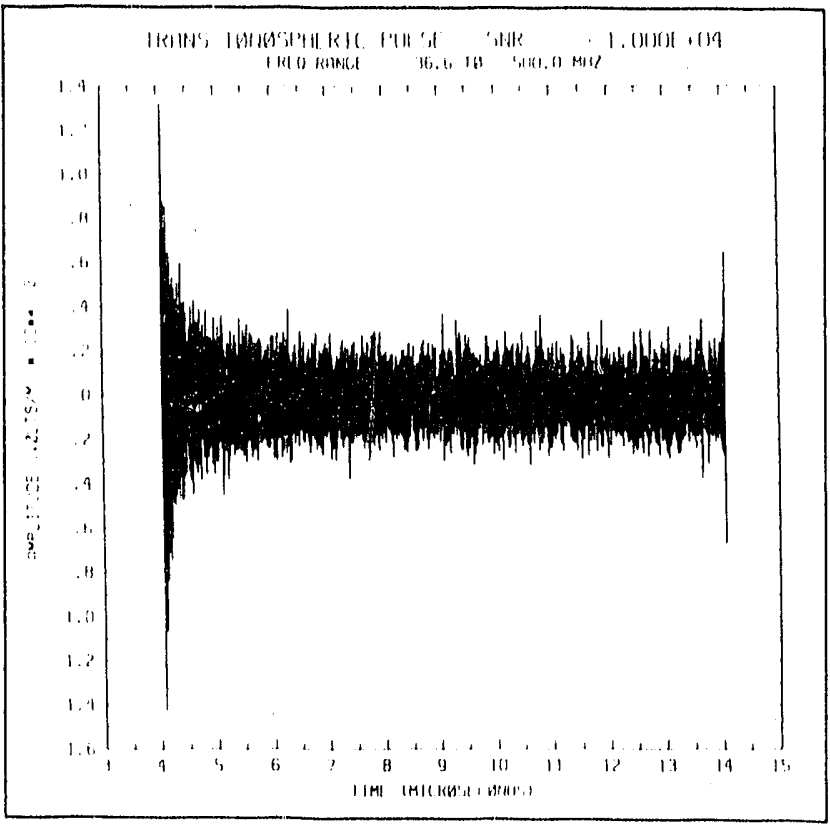

Figure $3 \mathrm{j}$ 


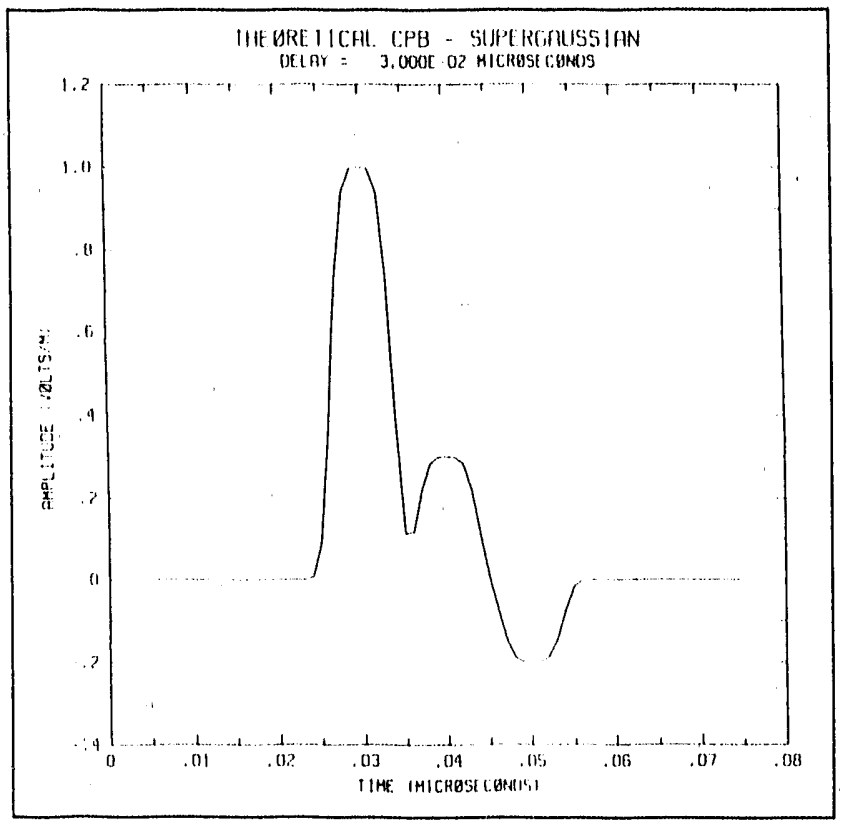

Figure 4a

Figure $4 b$

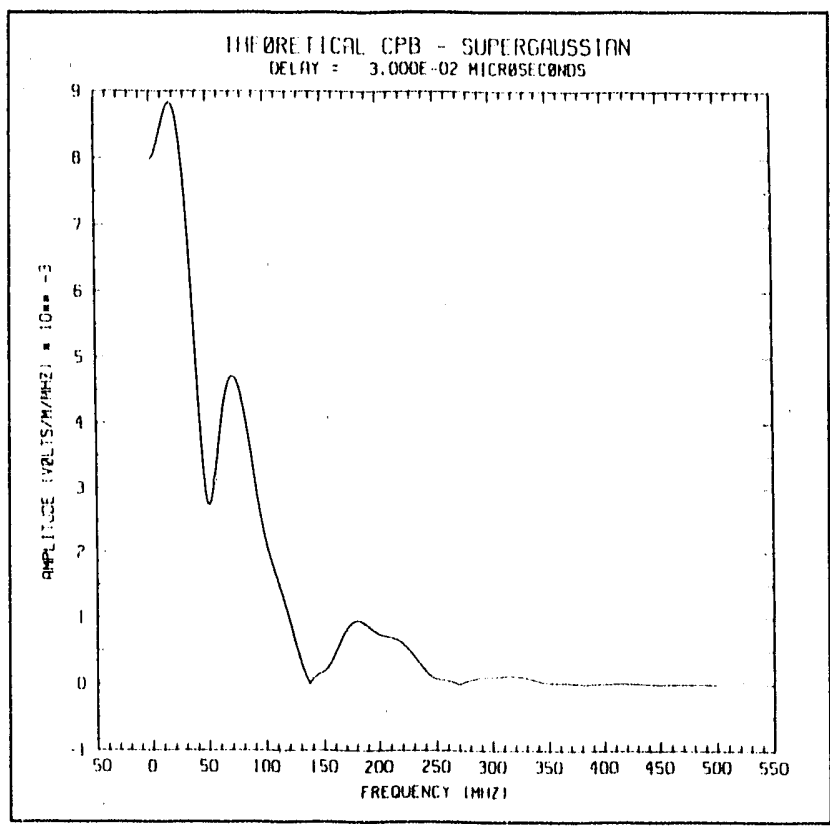

Figures $4 \mathbf{a}-\mathbf{j}$ (a) Temporal plot of the default super-Gaussian pulse. (b) FFT of the default super-Gaussian pulse. (c)-(f) Corresponding transionospheric signals for an TEC of $1 \times 10^{13} \mathrm{~cm}^{-2}$, an SNR limited by numerical noise $(70 \mathrm{~dB})$, and $F_{c o h}=\infty, 20,10$, and $1 \mathrm{MH} \%$, respectively. (g)-(h) Temporal plot of the noisy default delta function with an $S N R=100$ and the corresponding transionospheric signal for a TEC of $1 \times 10^{13} \mathrm{~cm}^{-2}, H_{c o h}=\infty$, and an SNR $=100$, respectively. (i)-(j) Temporal plot of the noisy default delta function with an $S N R=10,000$ and the corresponding transionospheric signal for a TEC of $1 \times 10^{.3} \mathrm{~cm}^{-2}, l^{\prime}$ coh $=\infty$, and an SNR $=10,(00)$, respectively. 


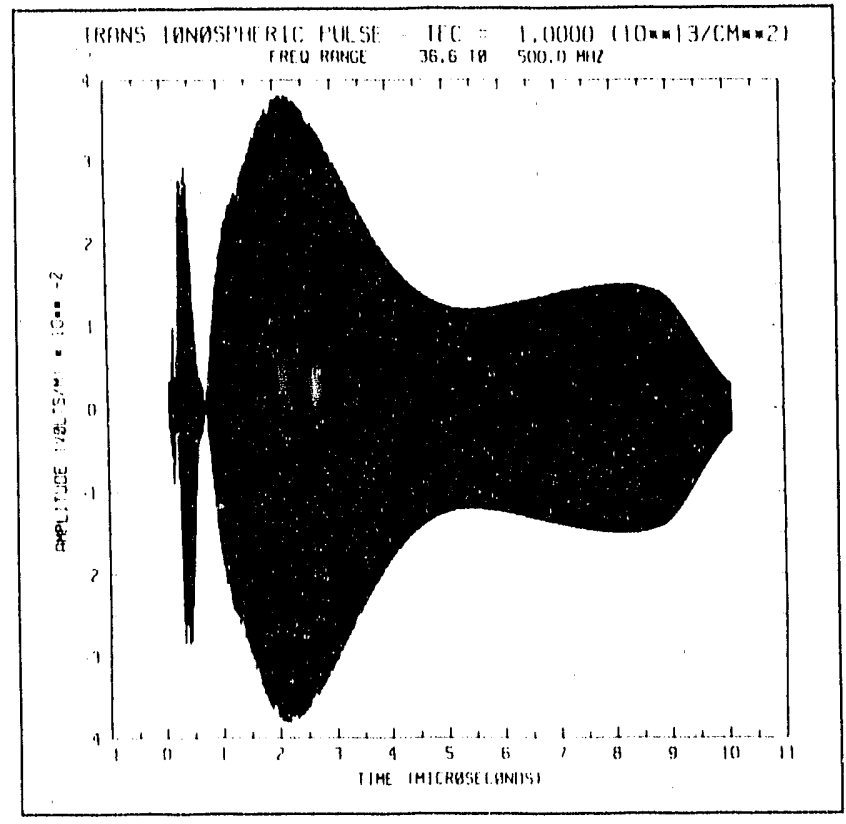

Figure $4 c$

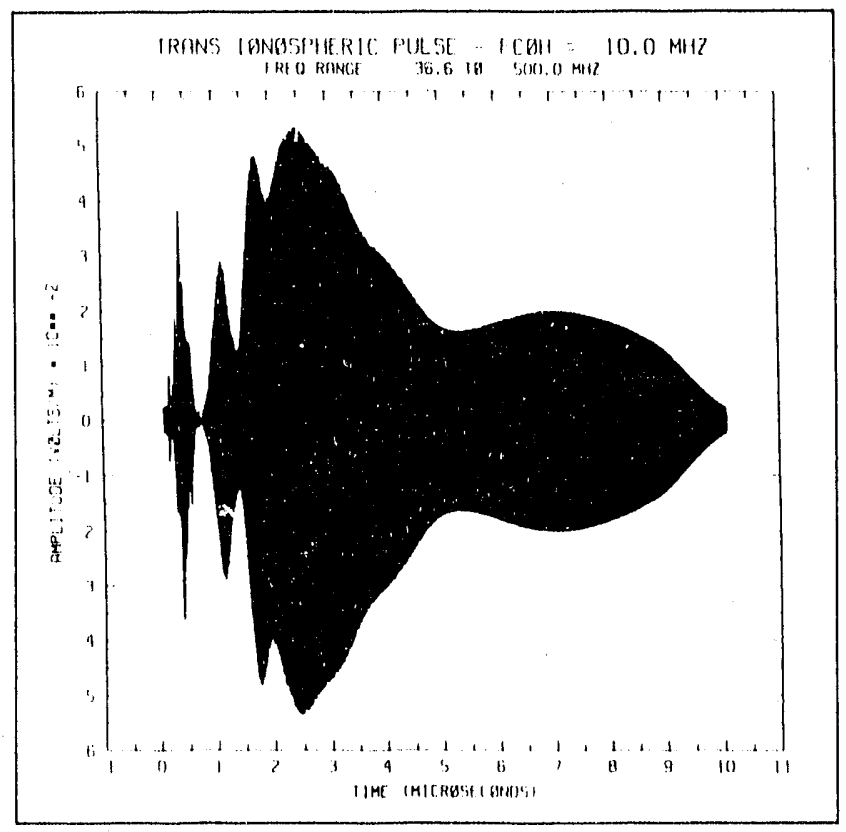

Figure te

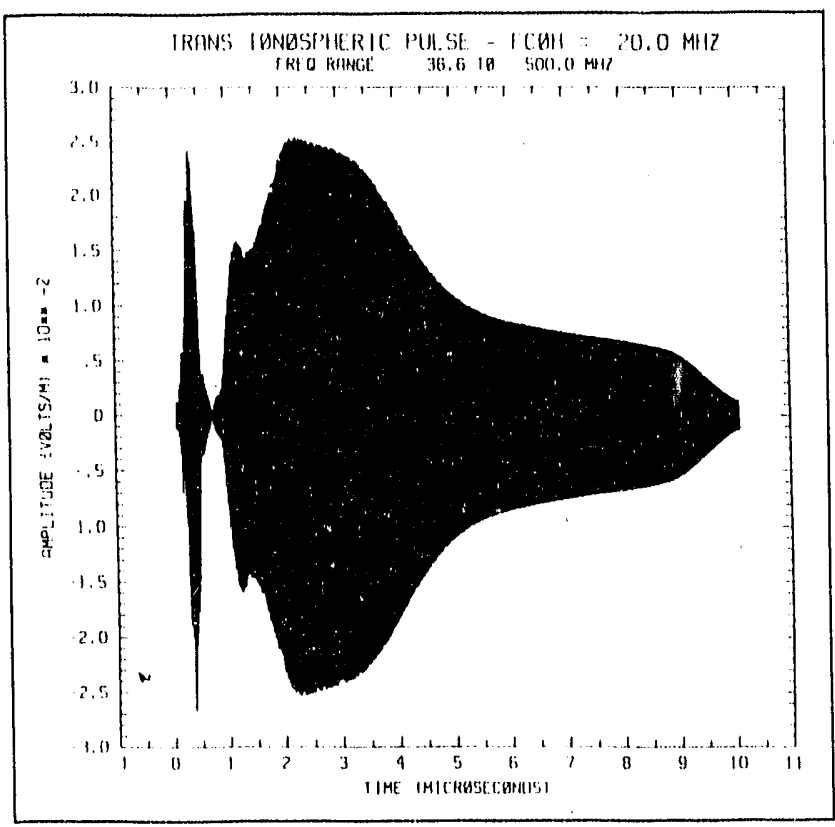

Figure Ad

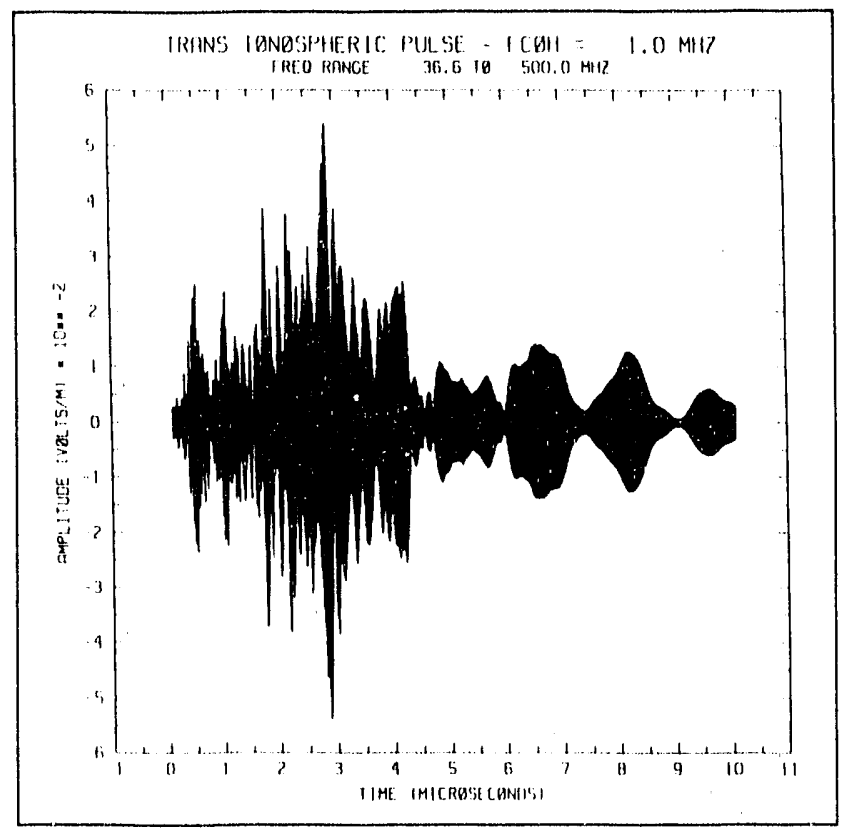

Figure $4 f$

51 


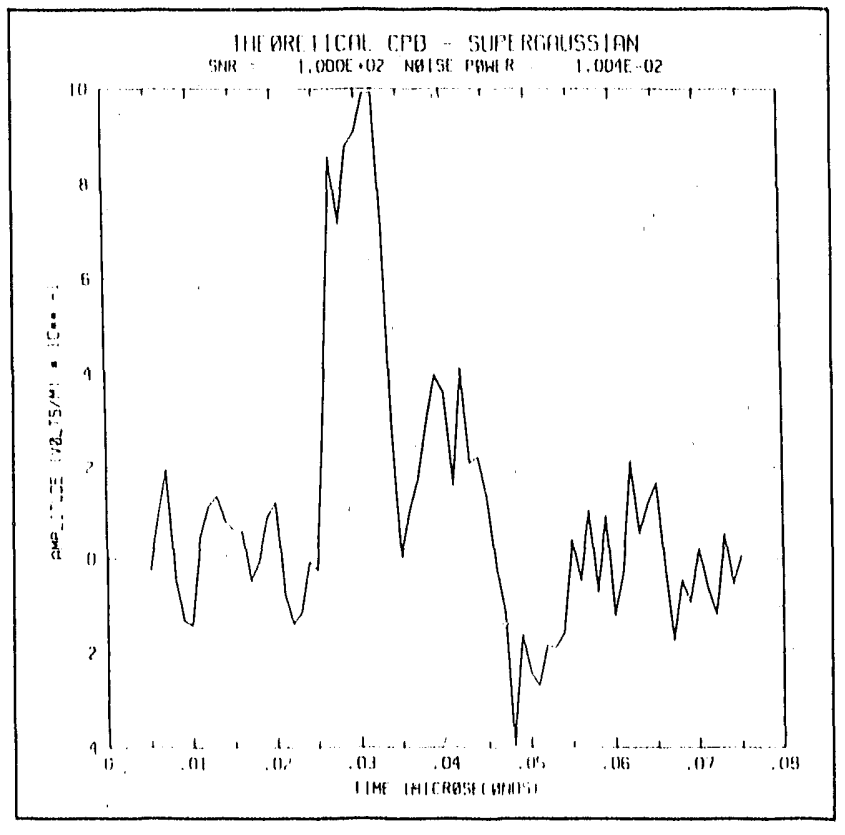

Figure $4 \mathrm{~g}$

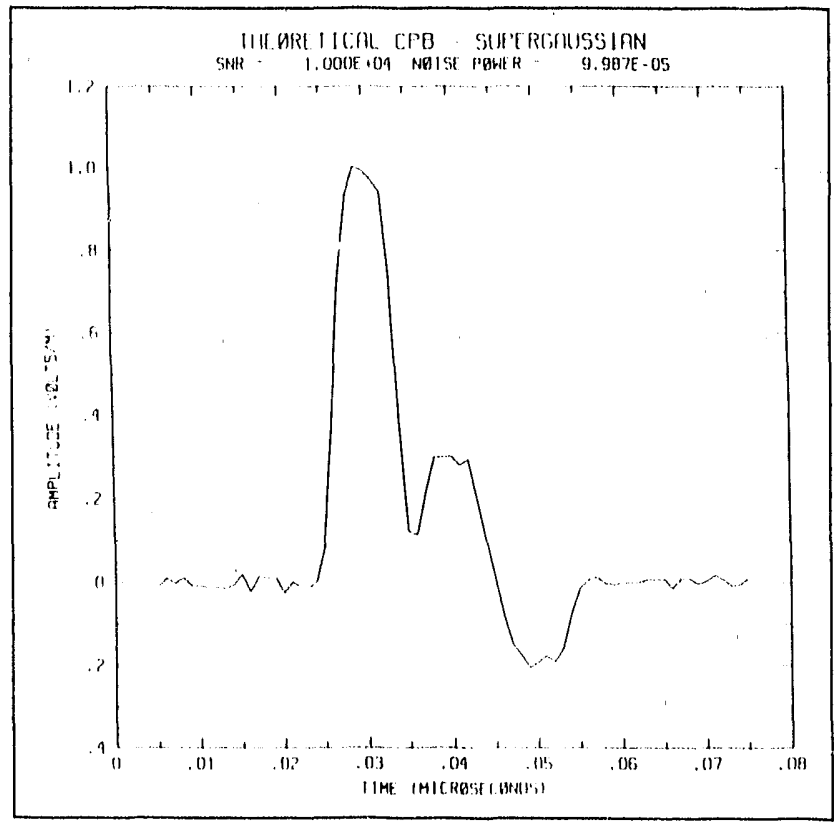

Figure 4i

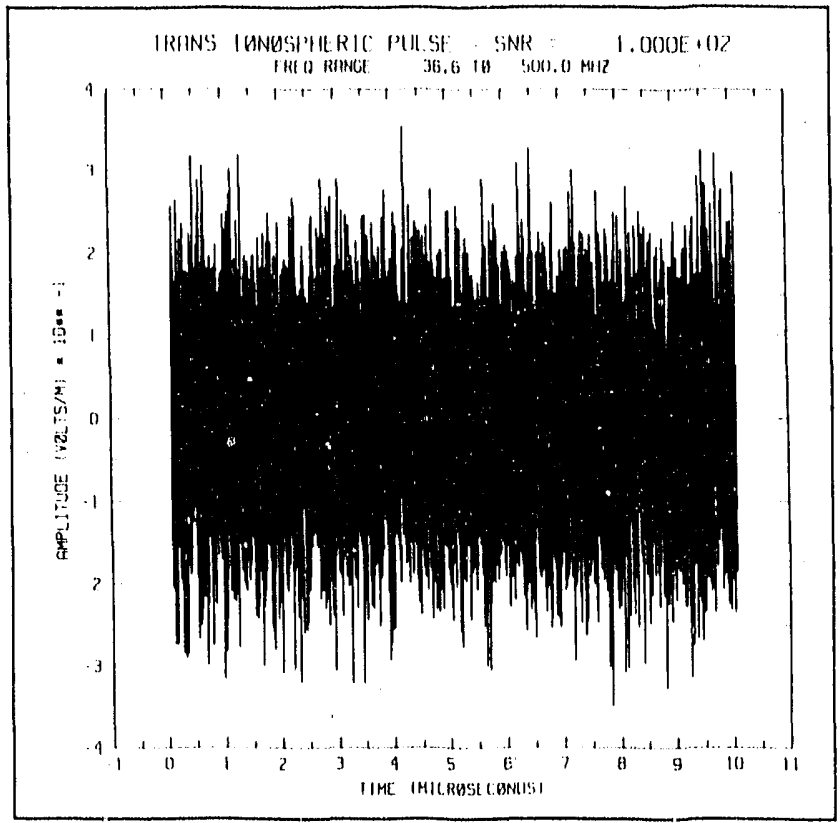

Figure $4 h$

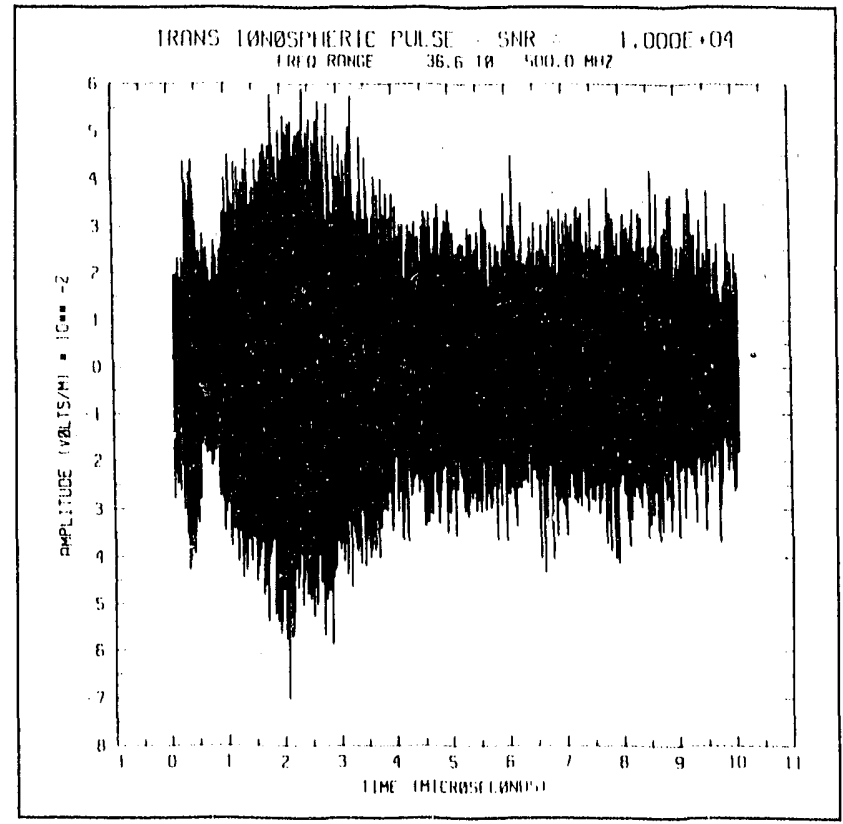

Figure $4 j$ 


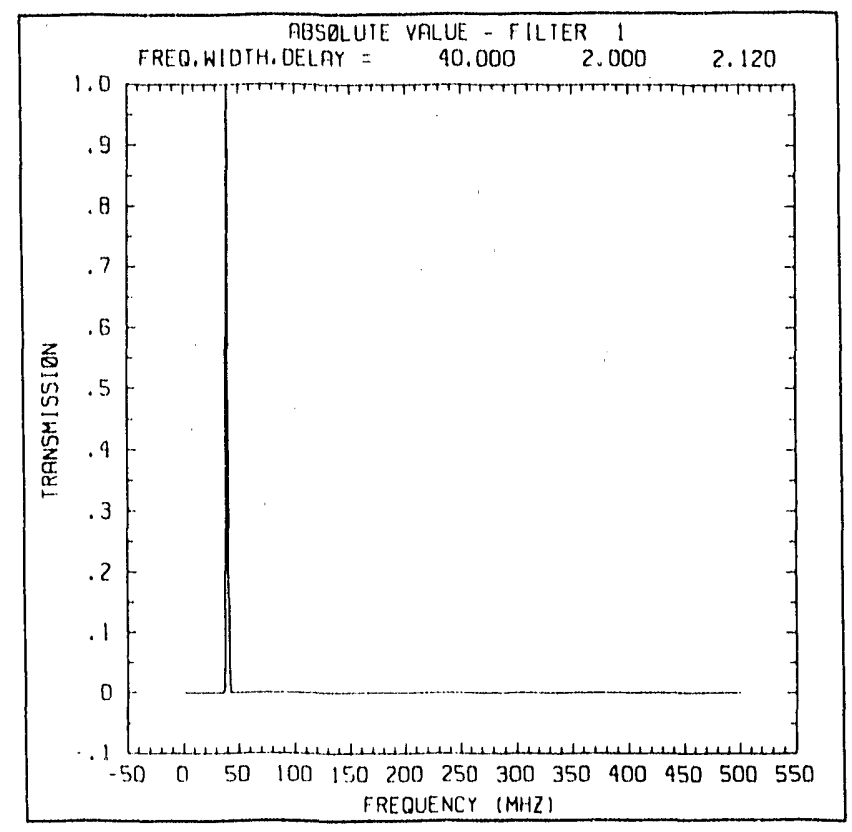

Figure 5a

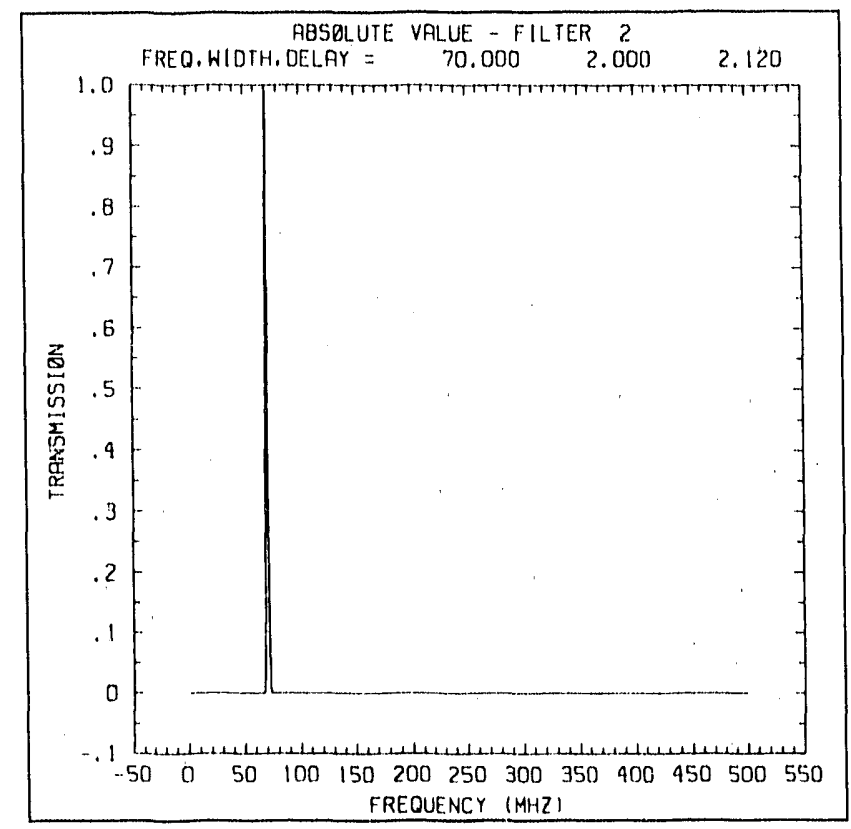

Figure 5b

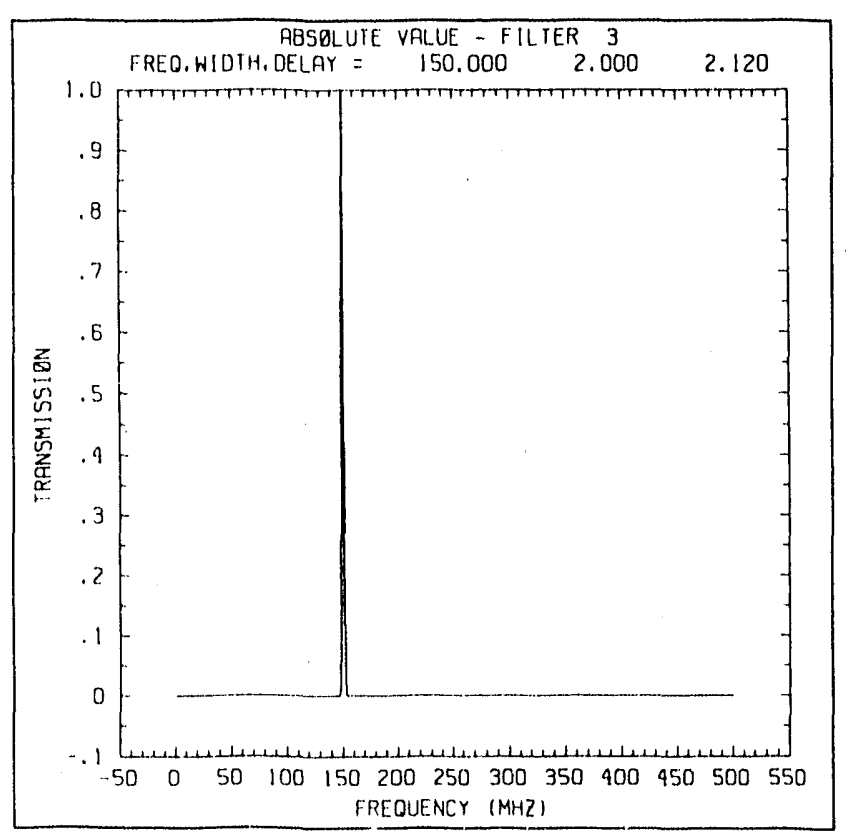

Figure 5c

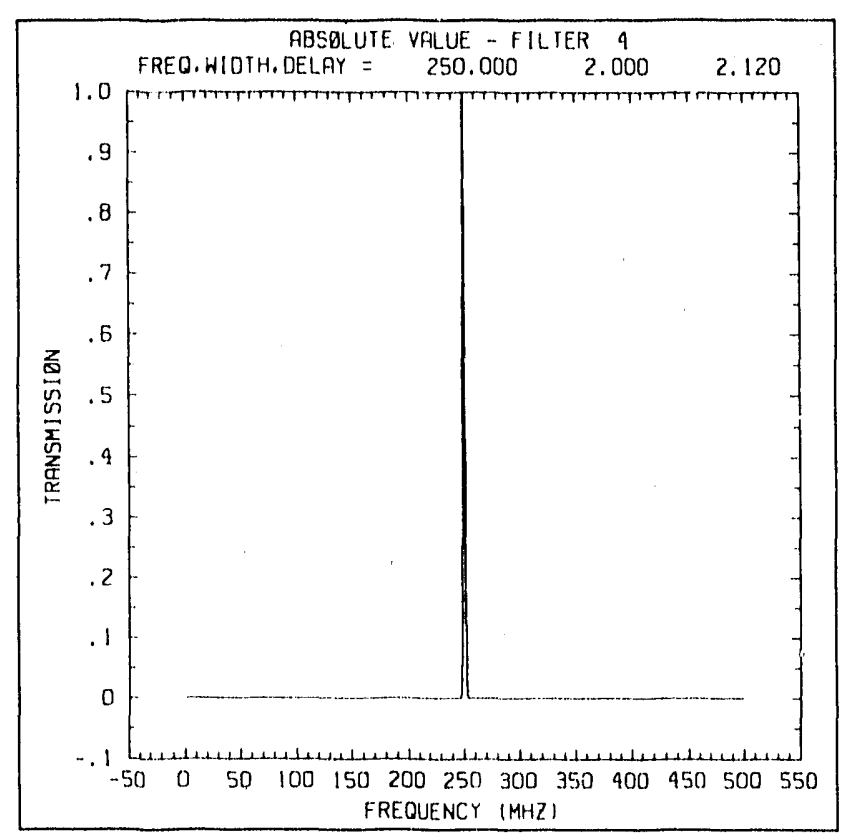

Figure 5d

Figures 5a-d (a)-(d) Transmission profiles for four Gatussian filters with 2-MHz bandwidths (FWHM) and central frequencies of $40,70,150$, and $250 \mathrm{MH} \%$, respectively. 


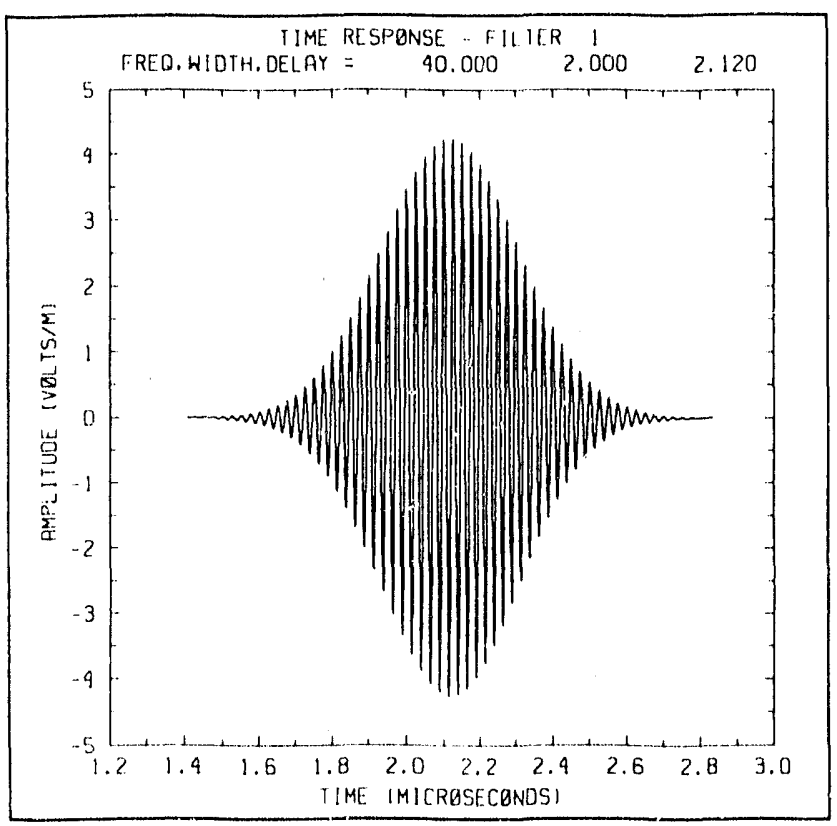

Figure 6a

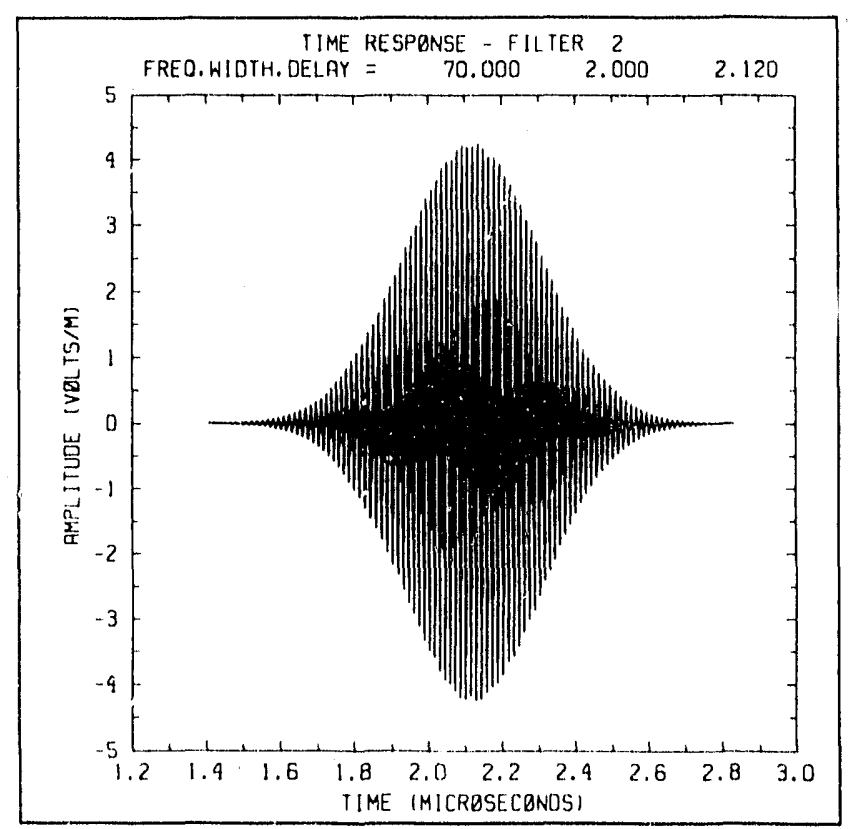

Figure 6b

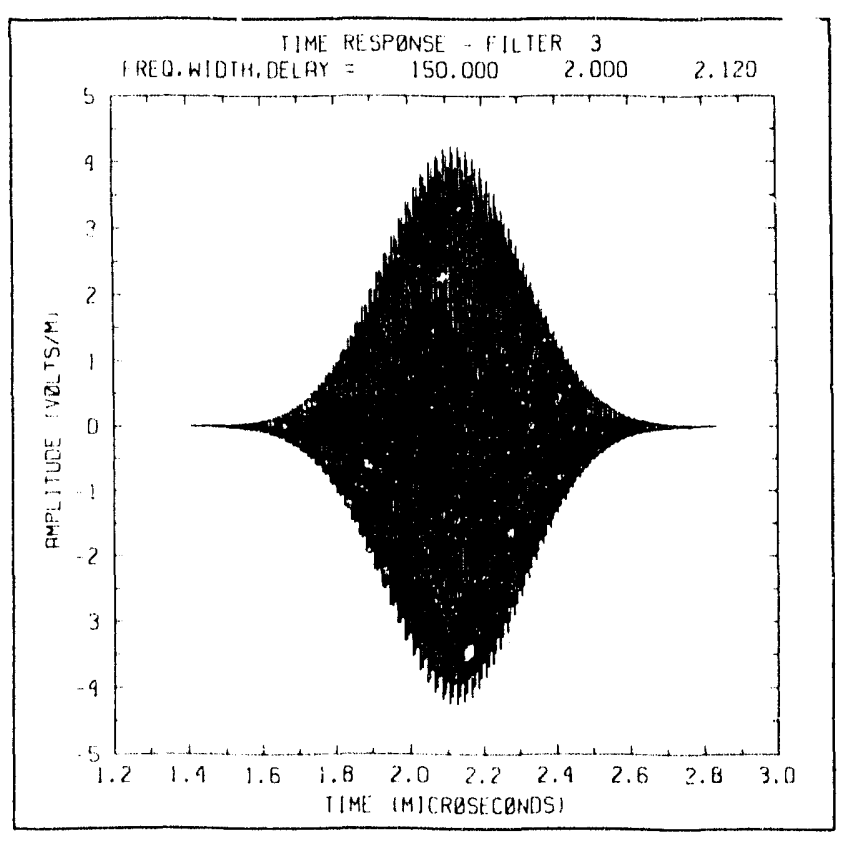

Figure 6c

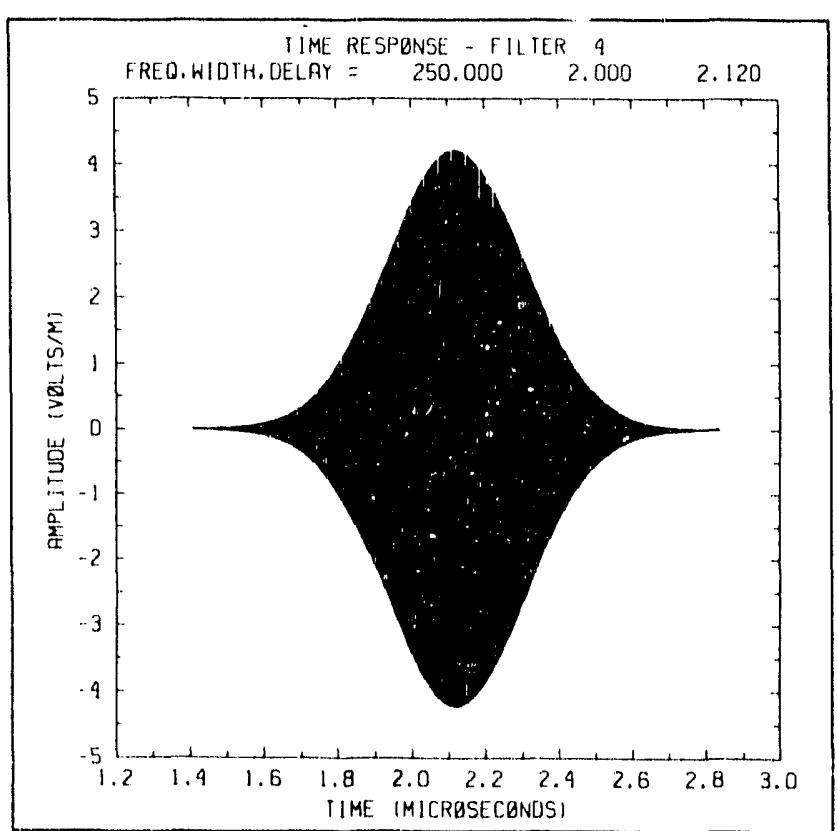

Figure 6d

Figures 6a-d (a)-(d) Impulse response functions for four (jaussian filters with 2-MHz bandwidths (FWHM) and central frequencies of 40,70, 150, and 250 MHz, respectively. Each filter has a built in time delay equal to approximately $2.12 \mu \mathrm{s}$. 


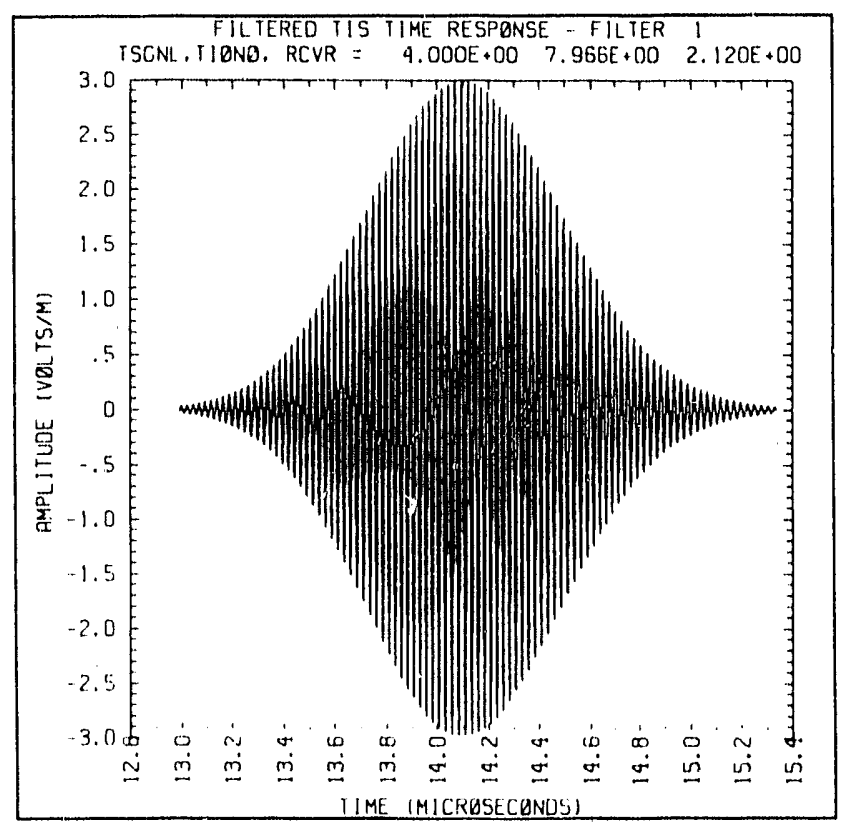

Figure 7a

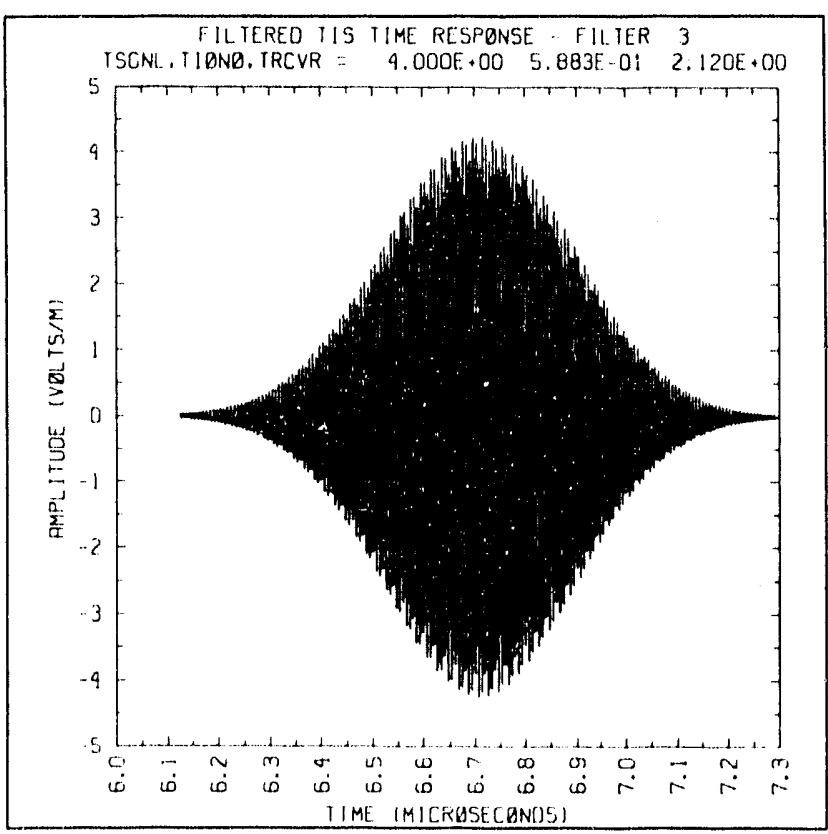

Figure $7 \mathrm{c}$

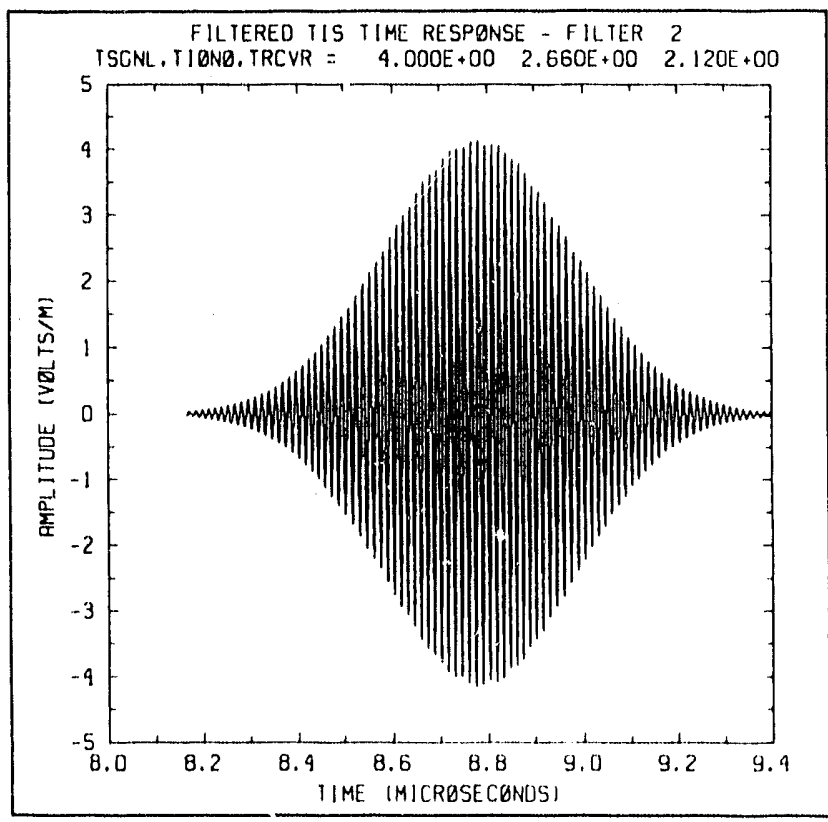

Figure $7 \mathrm{~b}$

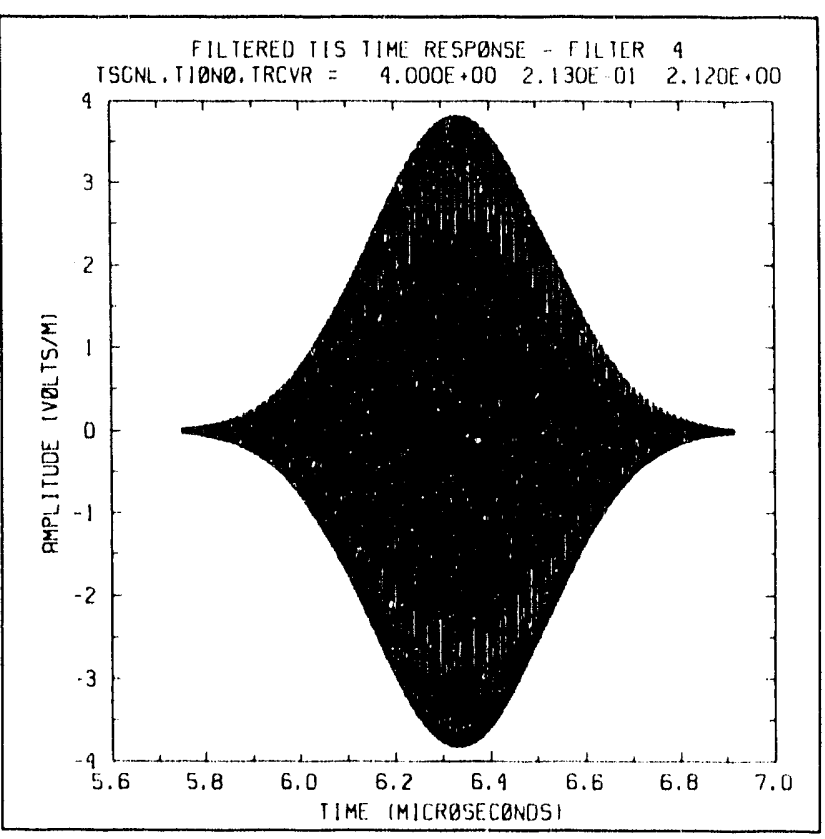

Figure 7d

Figures 7a-d (a)-(d) Filtered transionospheric signals obtained for the default delta function with the four Gaussian filters shown in Figures $5 \mathrm{a}-\mathrm{d}$, respectively. A TEC of $1 \times 10^{13} \mathrm{~cm}{ }^{2}, F, F_{1}=x$, and an SNR of $70 \mathrm{~dB}$ were assurned. 


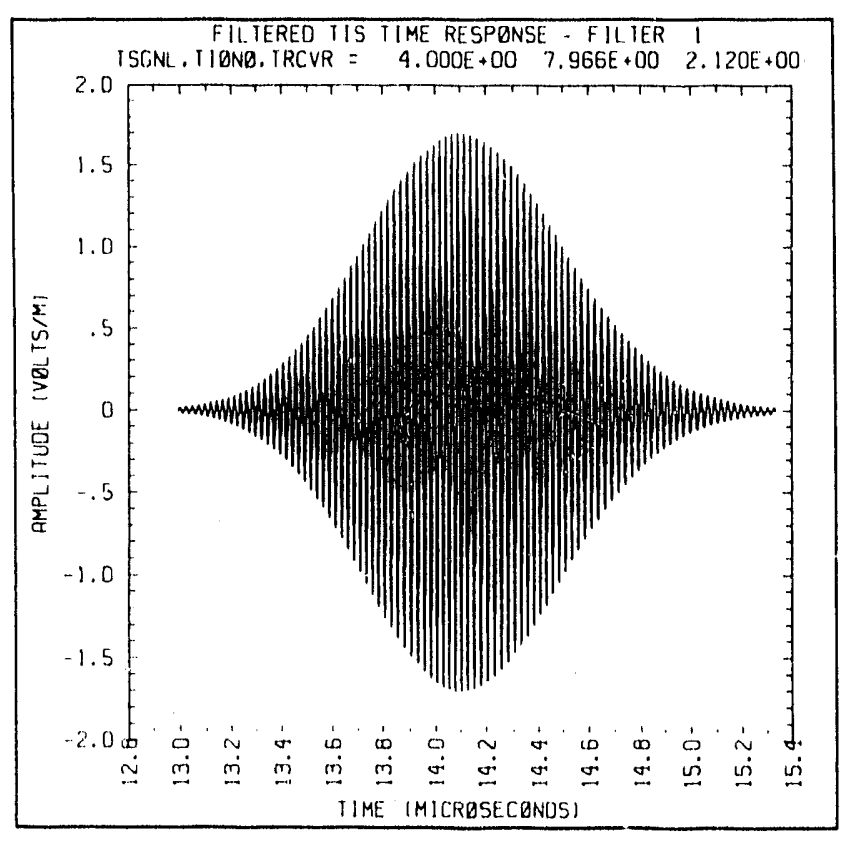

Figure 8a

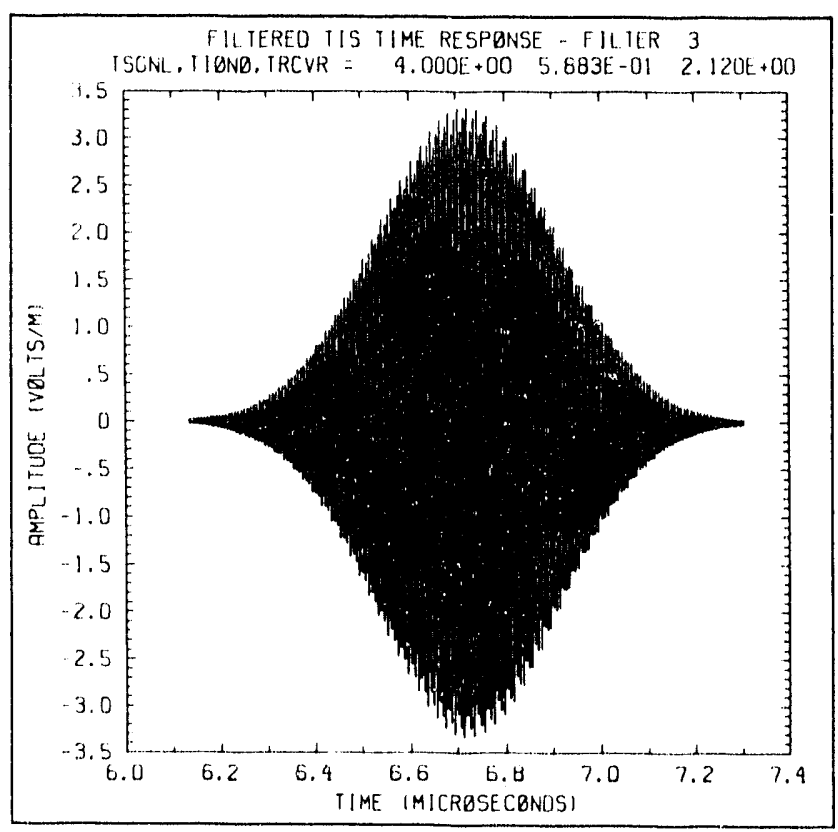

Figure 8c

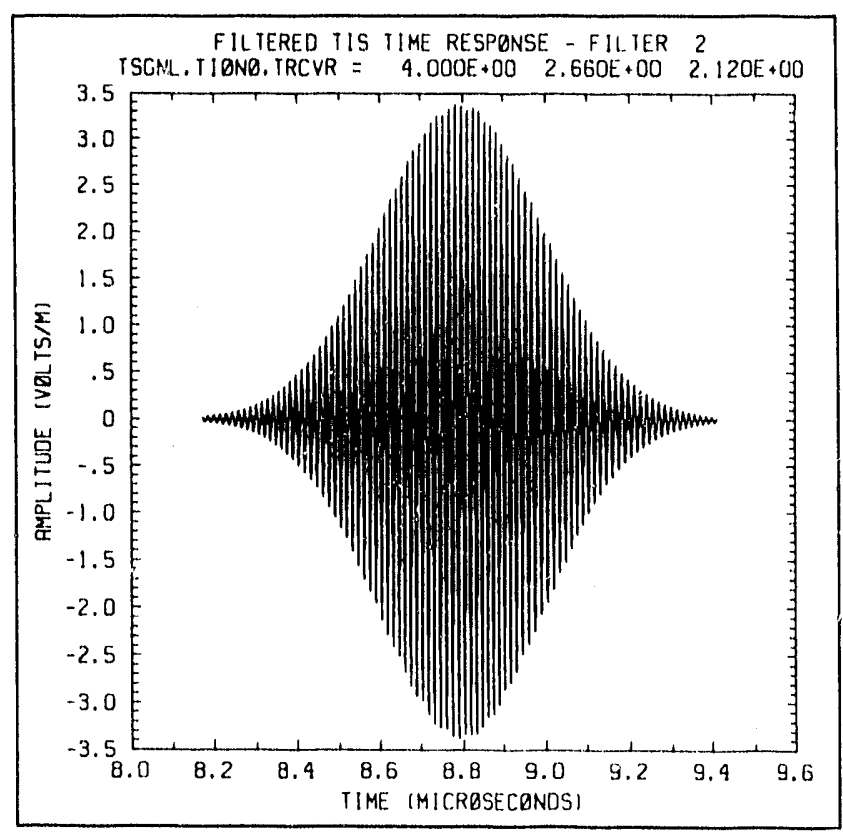

Figure 8b

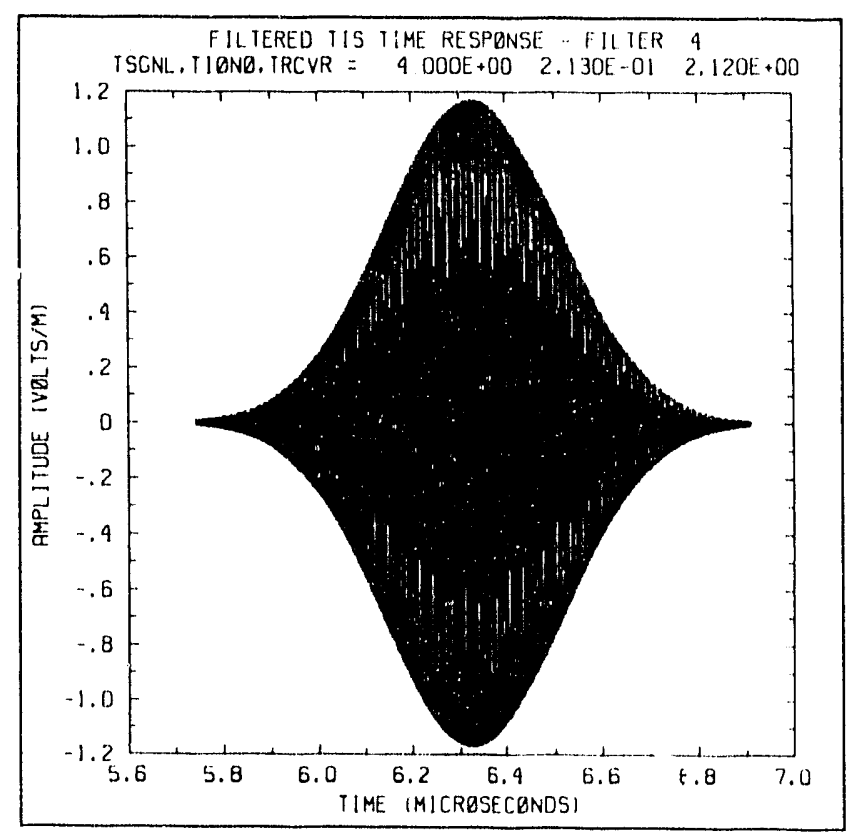

Figure 8d

Figures 8a-d (a)-(d) Filtered transionospheric signals obtained for the default delta function with the four (iaussian filters shown in Figures $5 \mathrm{a}-\mathrm{d}$, respectively. A TEC of $1 \times 10^{13} \mathrm{~cm}^{2}, F_{10 h}=20 \mathrm{MHz}$, and an SNR of $70 \mathrm{~dB}$ were assumed. 


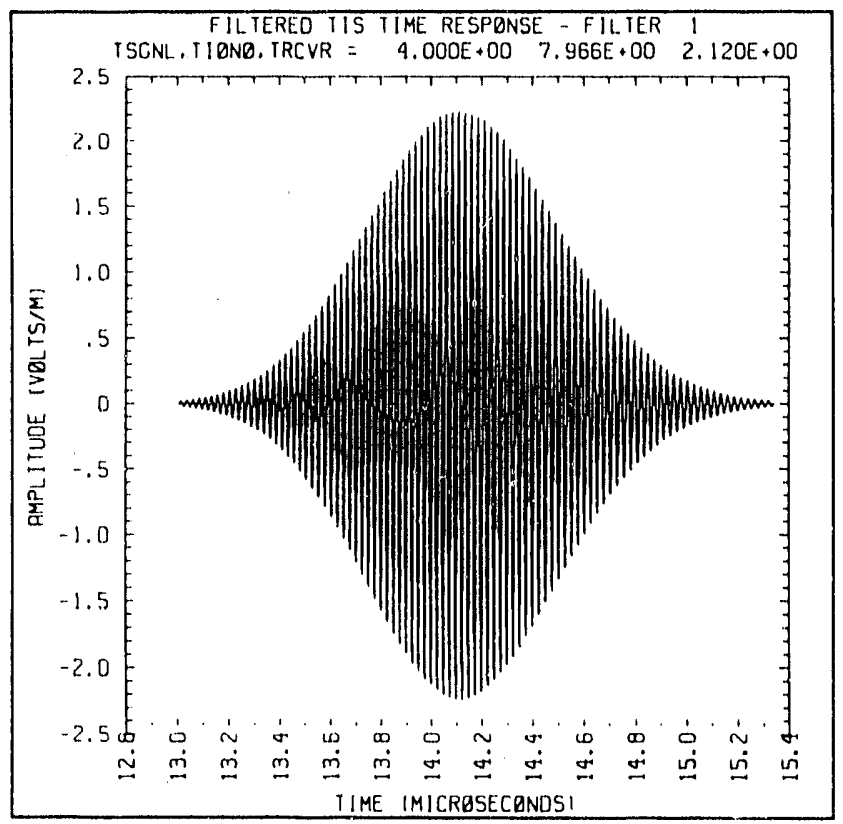

Figure 9a

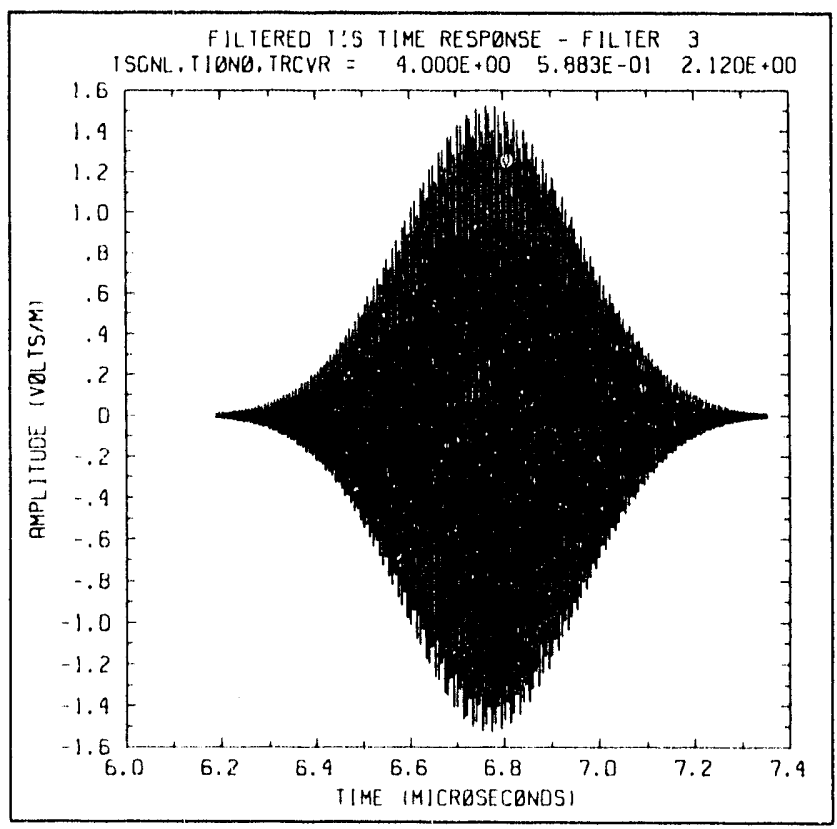

Figure 9c

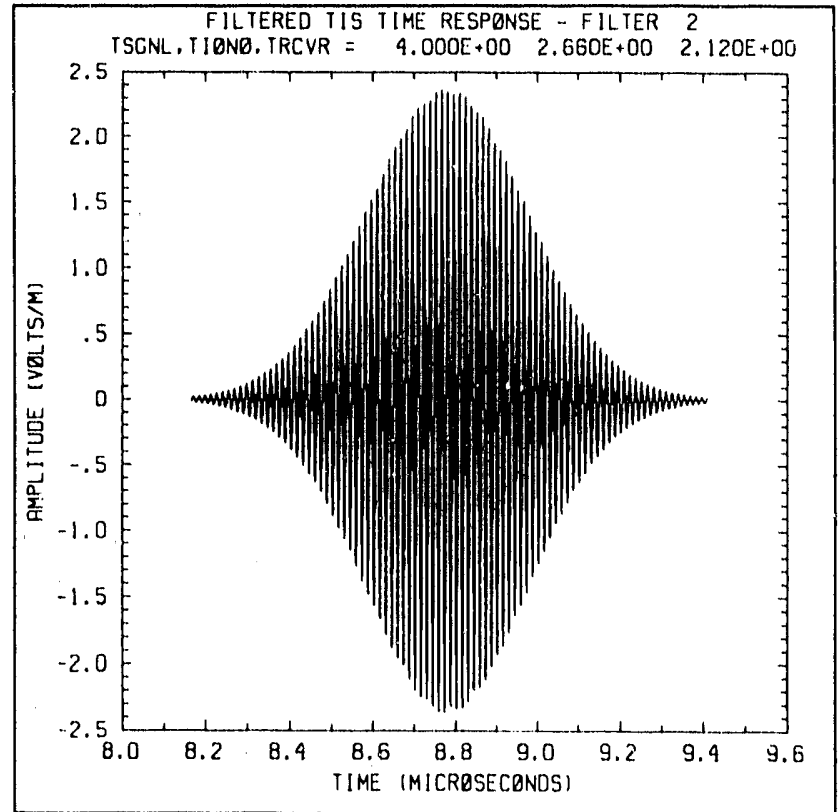

Figure 9b

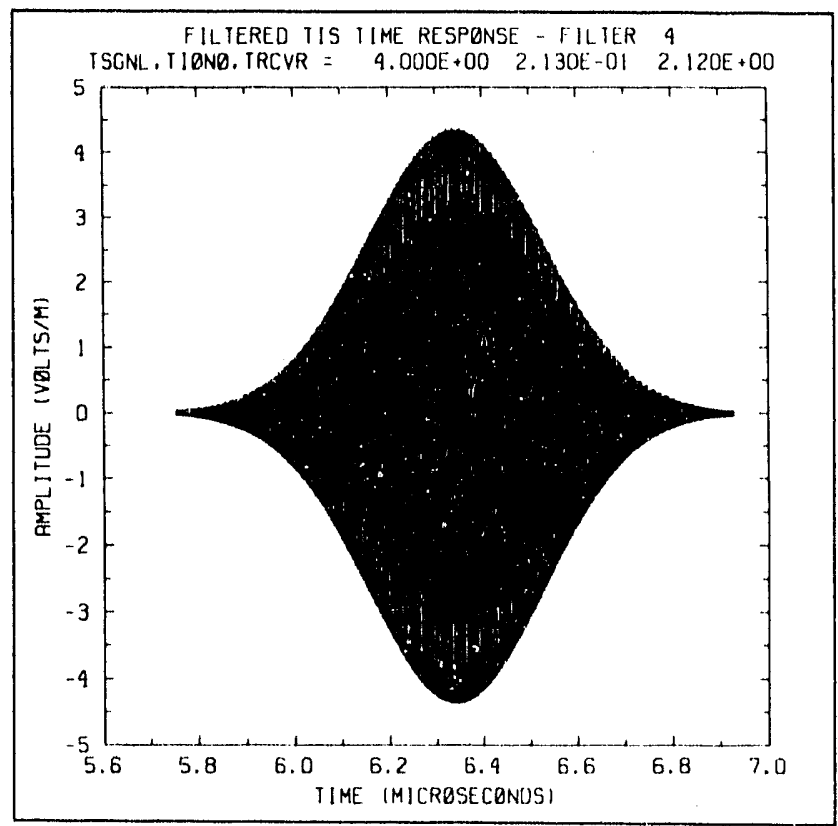

Figure 9d

Figures 9a-d (a)-(d) Filtered transionospheric signals obtained for the default delta function with the four Gaussian filters shown in Figures 5a-d, respectively. A TEC of $1 \times 10^{13} \mathrm{~cm}^{-2}, F_{c, 1}=10 \mathrm{MH} /$, and an SNR of $70 \mathrm{~dB}$ were assumed. 


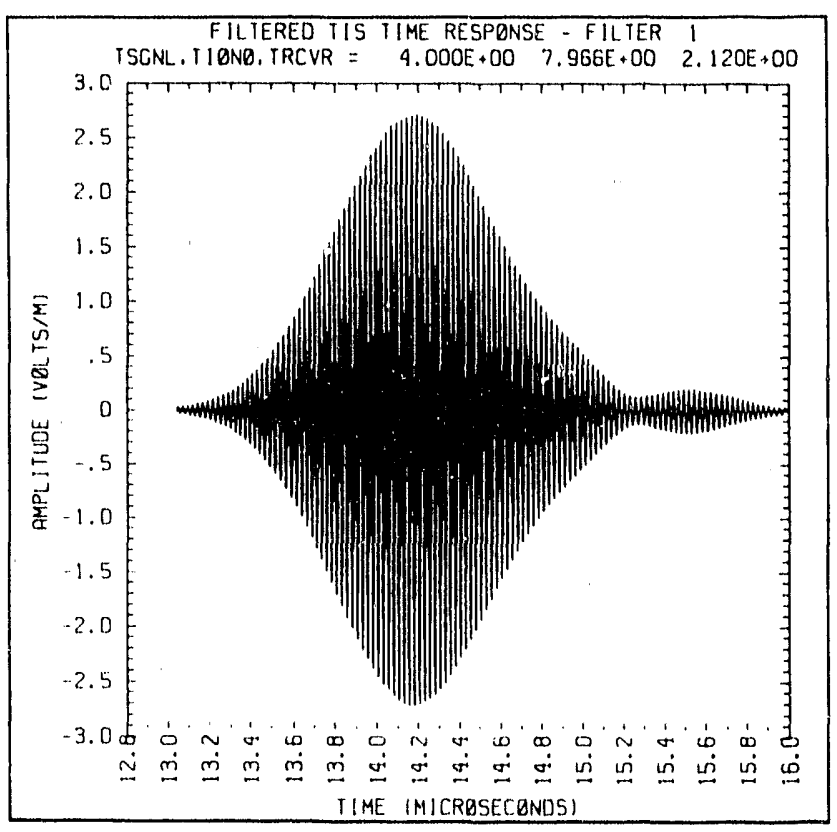

Figure 10a

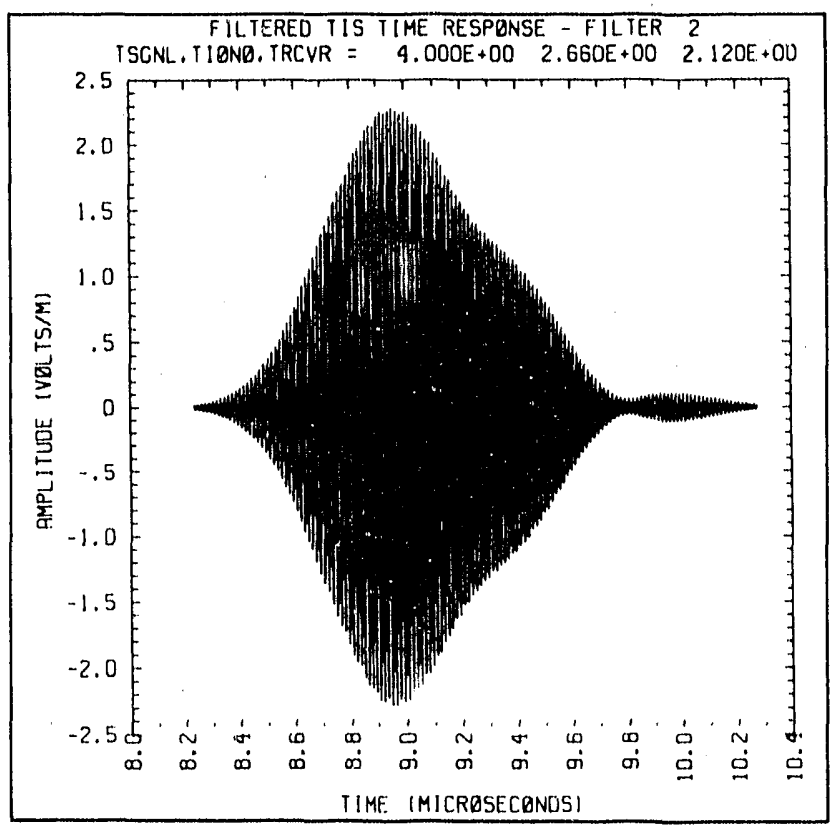

Figure 10b

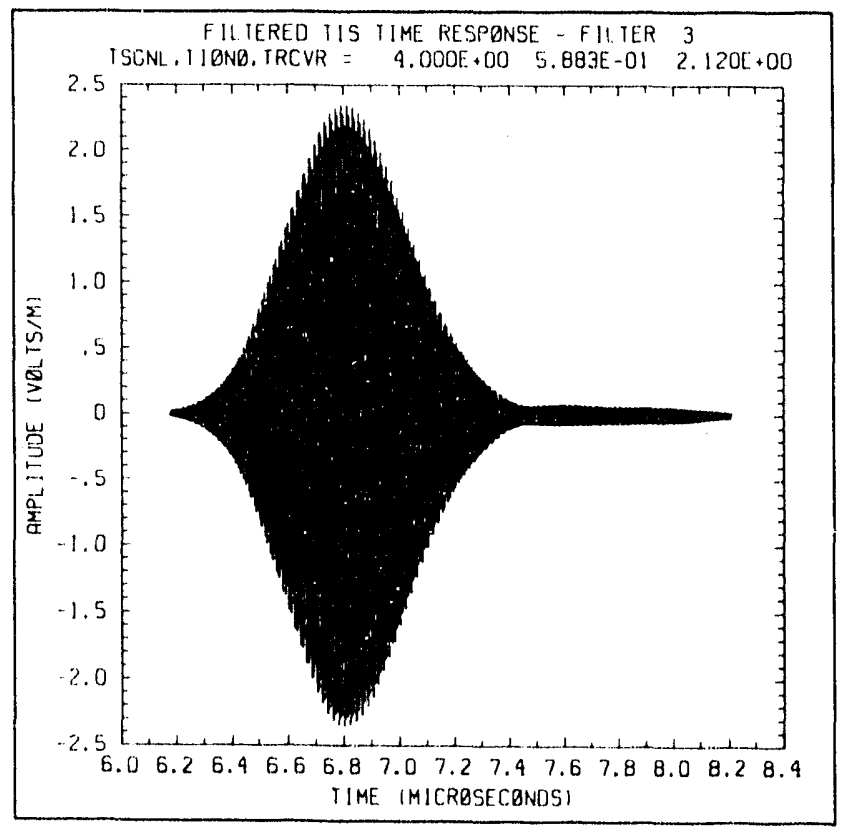

Figure 10c

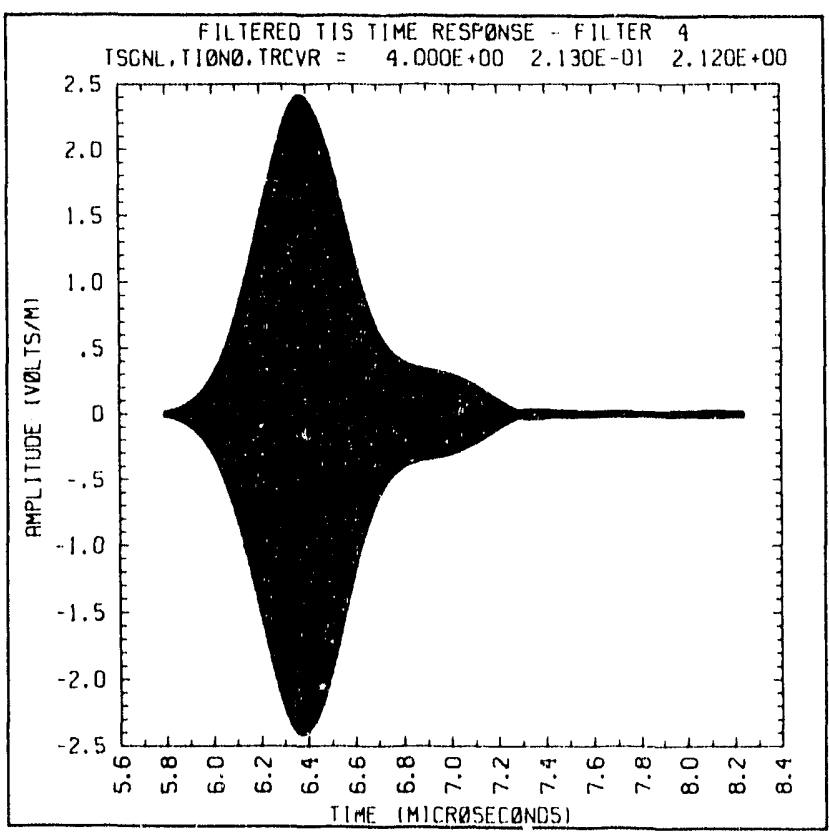

Figure 10d

Figures 10a-d (a)--(d) Filtered transionospheric signals obtained for the default delta function with the four Gaussian filters shown in Figures $5 \mathrm{a}-\mathrm{d}$, respectively. A TEC of $1 \times 10^{1: 3} \mathrm{~cm}{ }^{2}, F \mathrm{coh}=1 \mathrm{MHz}$, and an SNR of $70 \mathrm{~dB}$ were assumed. 


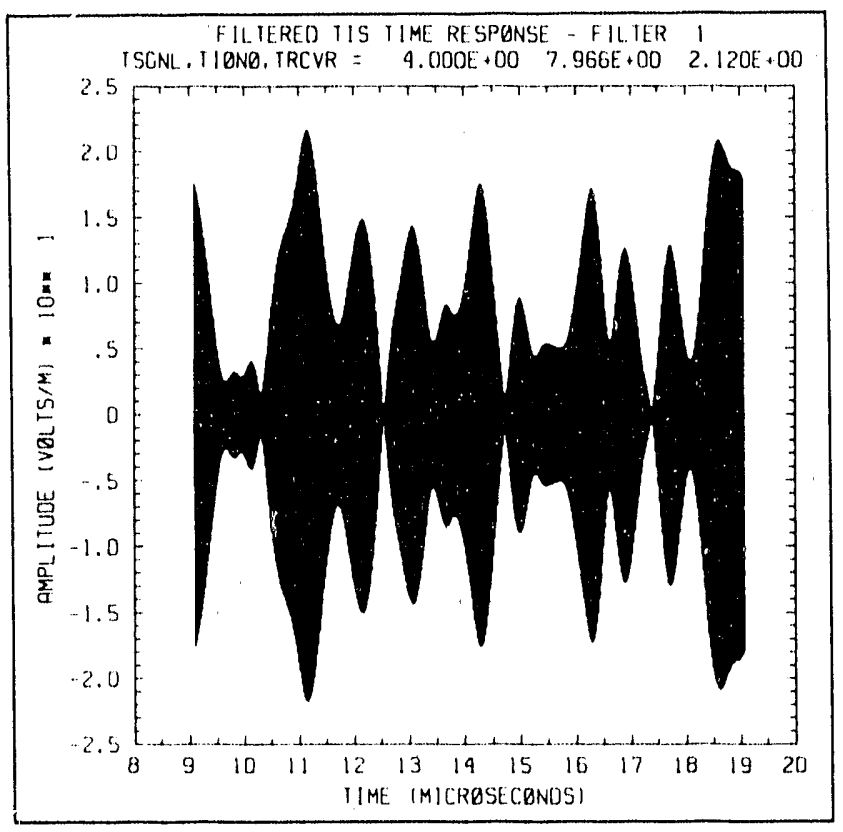

Figure 11 a

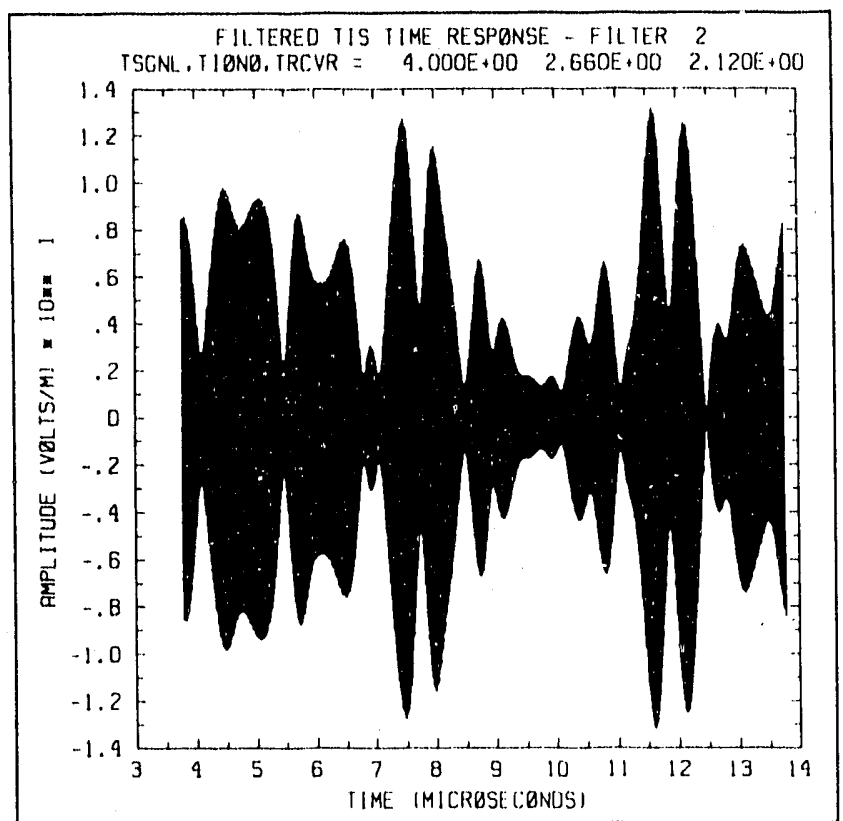

Figure $11 \mathrm{~b}$

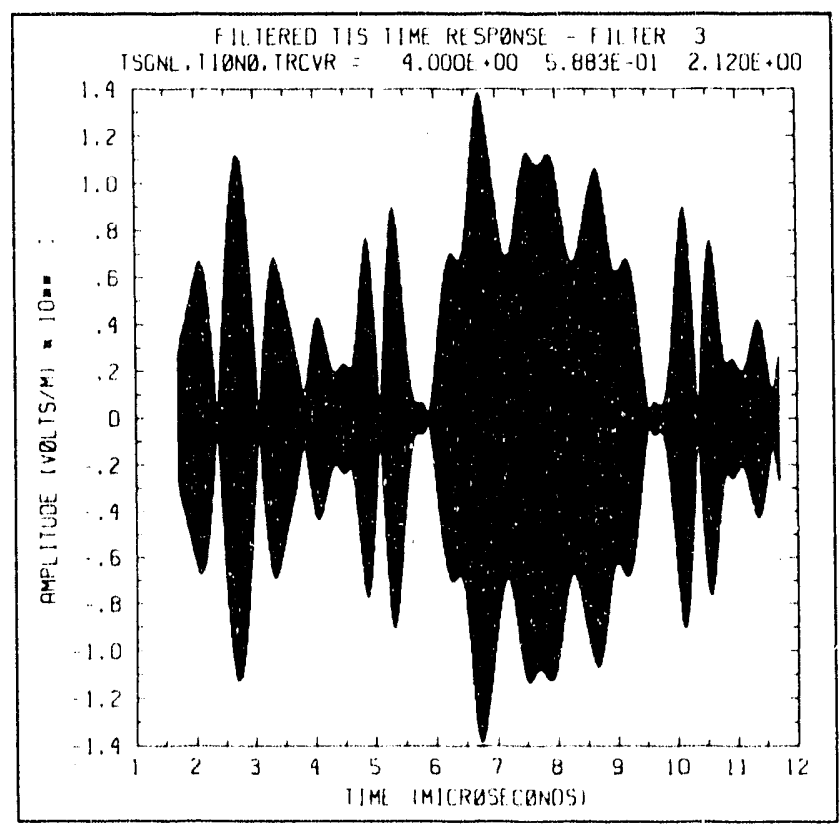

Figure $11 \mathrm{c}$

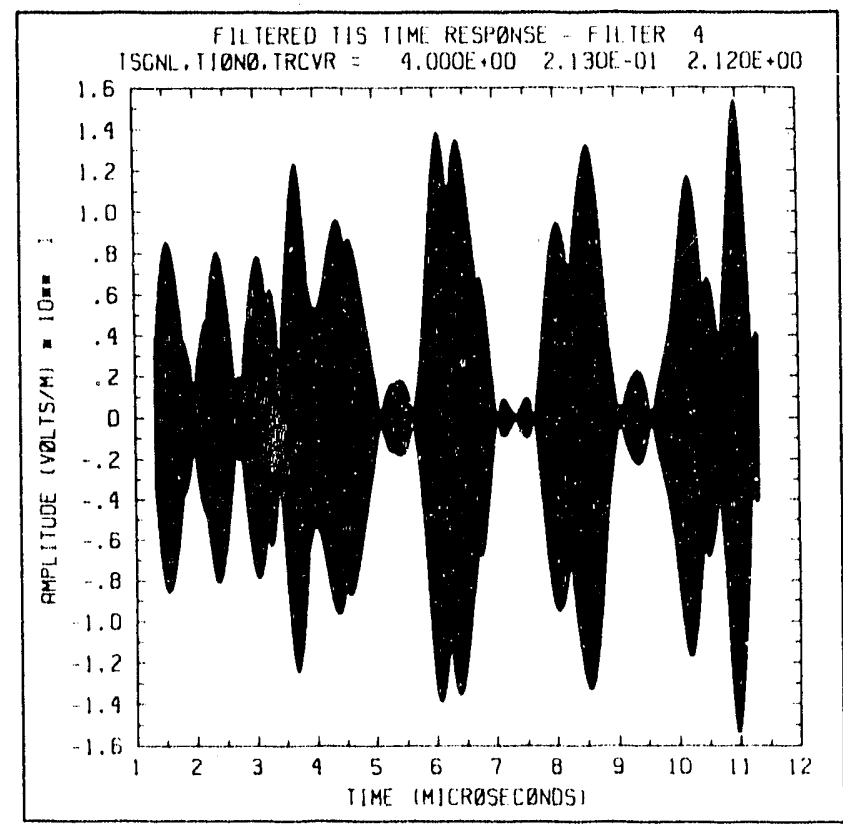

Figure 11d

Figures 1la-d (a) (d) filtered transionospheric signals obtained for the default delta function with the four Gaussian filters shown in Figures .5ad, respectively. A TEC of $1 \times 10^{13} \mathrm{~cm}^{2}, F r_{1}=x$, and an SNR of I(k) were assumed. 


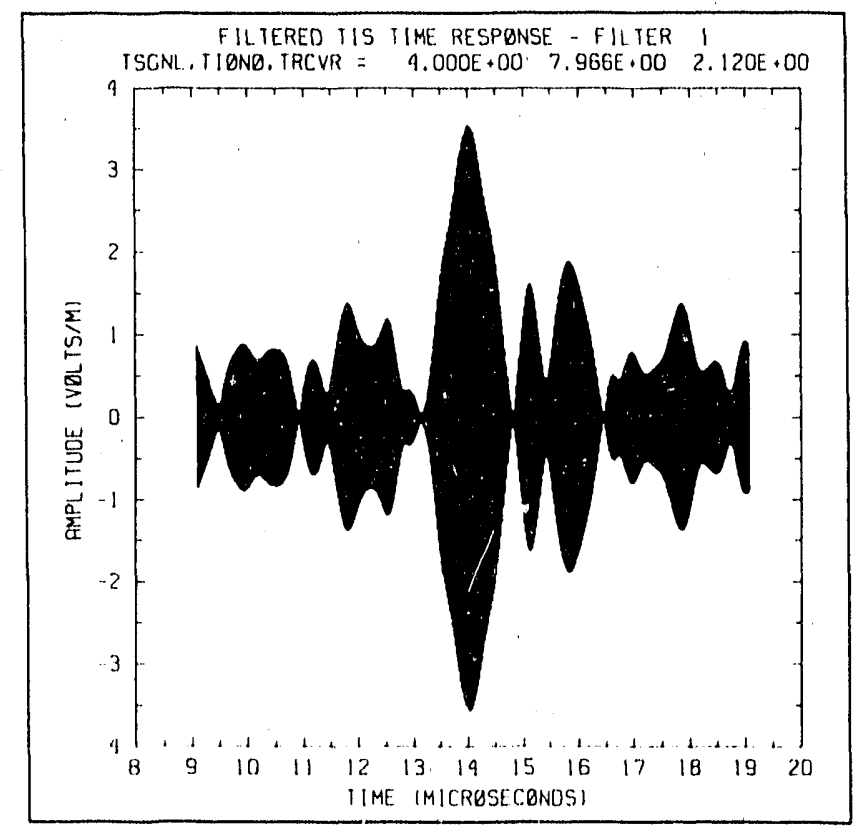

Figure 12a

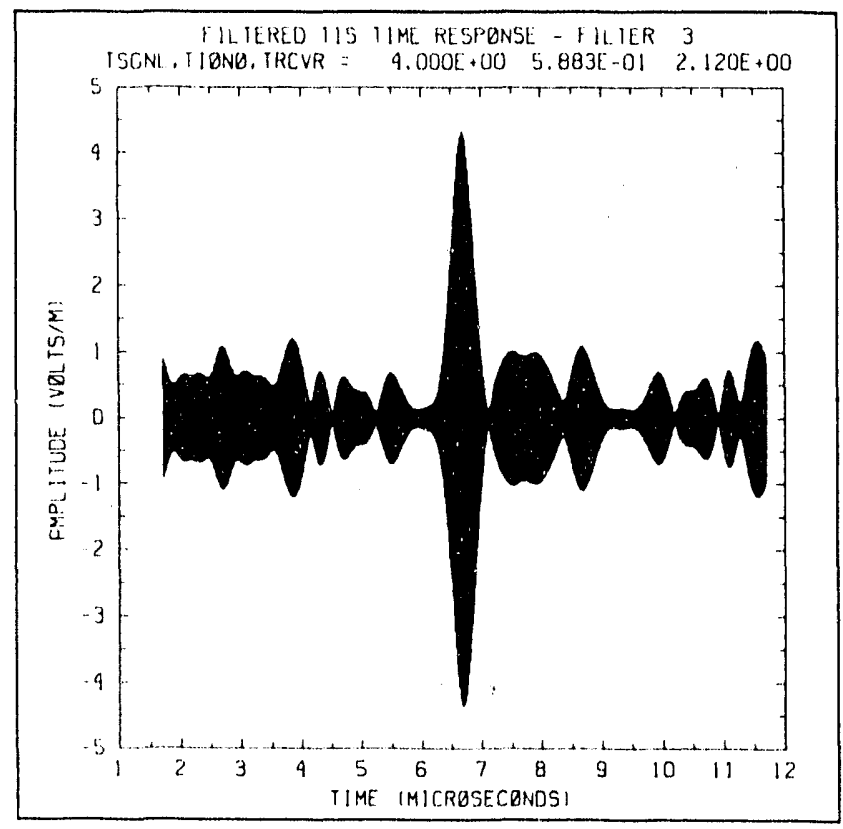

Figure 12c

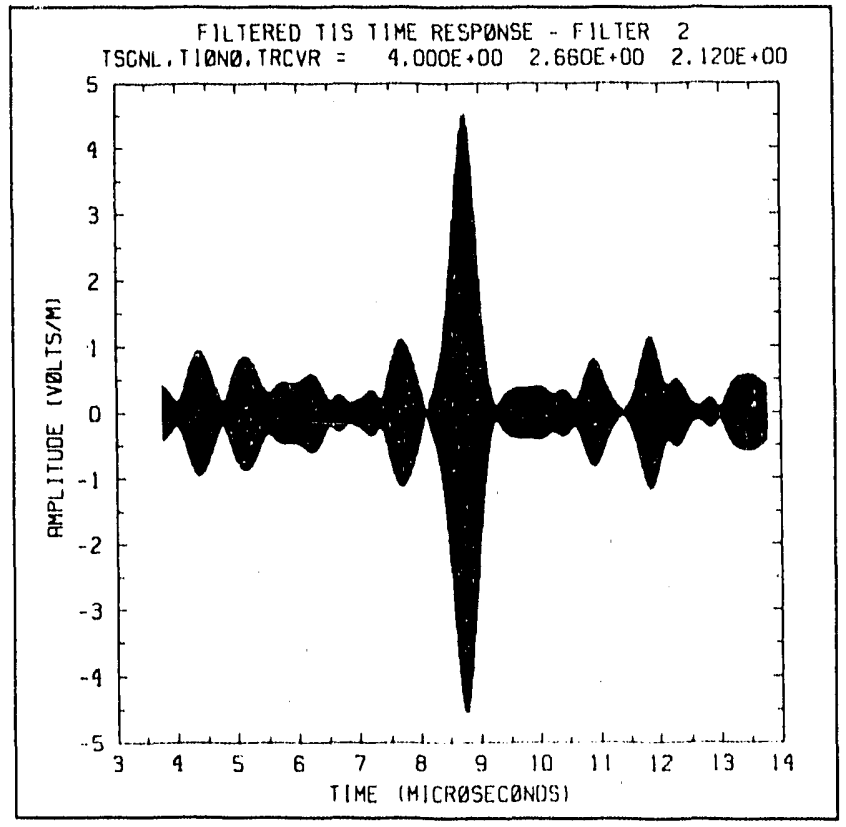

Figure 12b

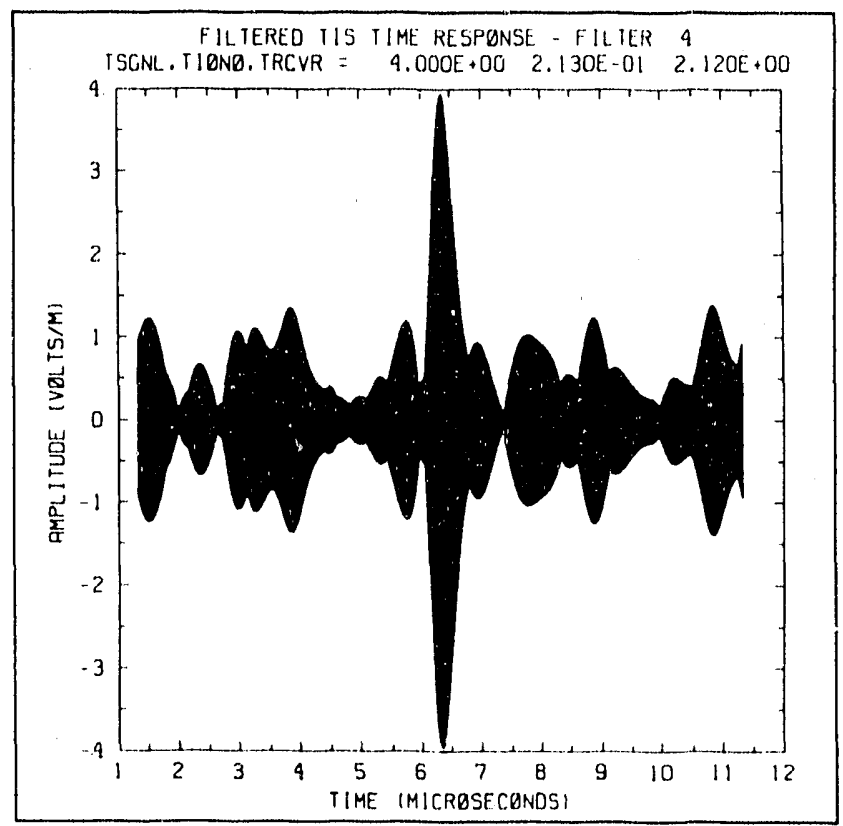

Figure 12d

Figures 12a-d (a)-(d) Filtered transionospheric signals obtained for the default delta function with the four Gaussian filters shown in Figures $5 \mathrm{~d}-\mathrm{d}$, respectively. A TEC of $1 \times 10^{13} \mathrm{~cm}^{-2}$. Fros $=x$, and an SNR of $10,()())$ were assumed. 


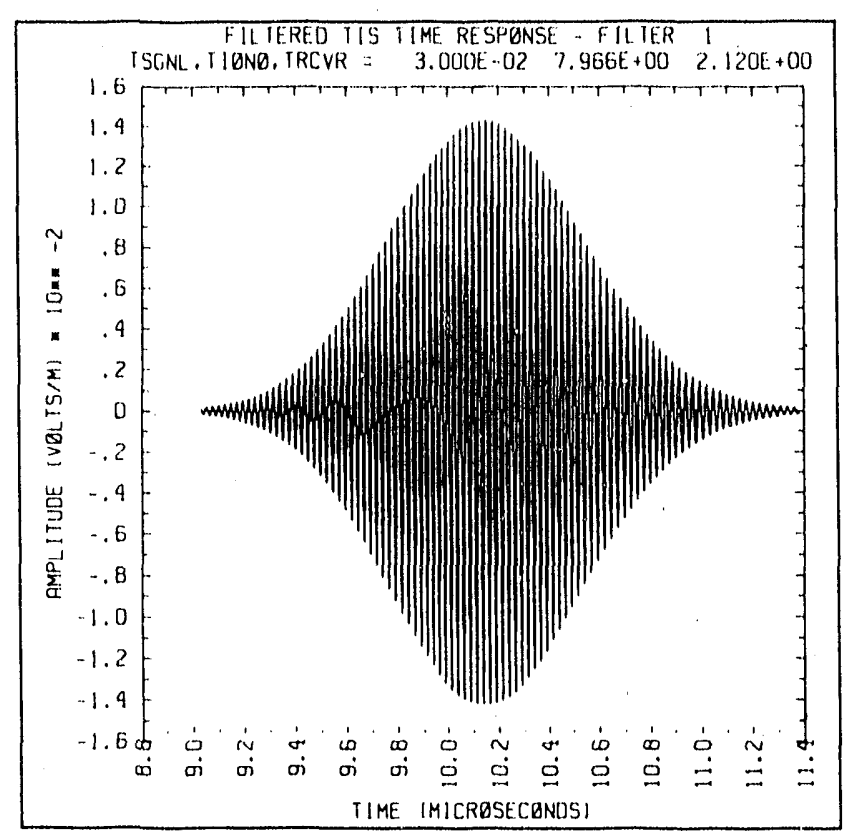

Figure 13a

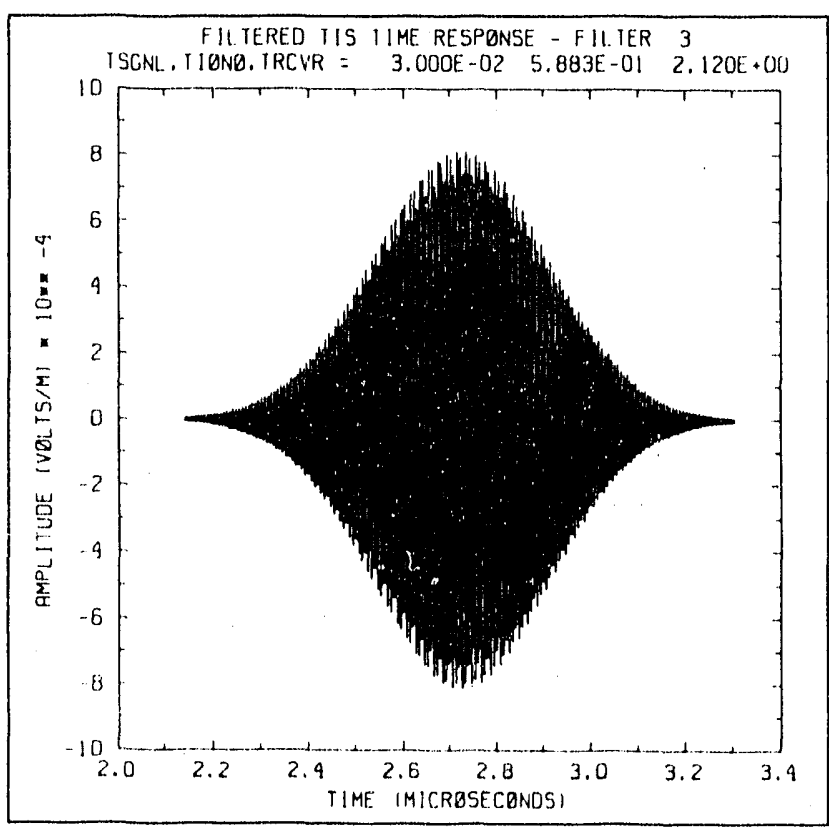

Figure $13 \mathrm{c}$

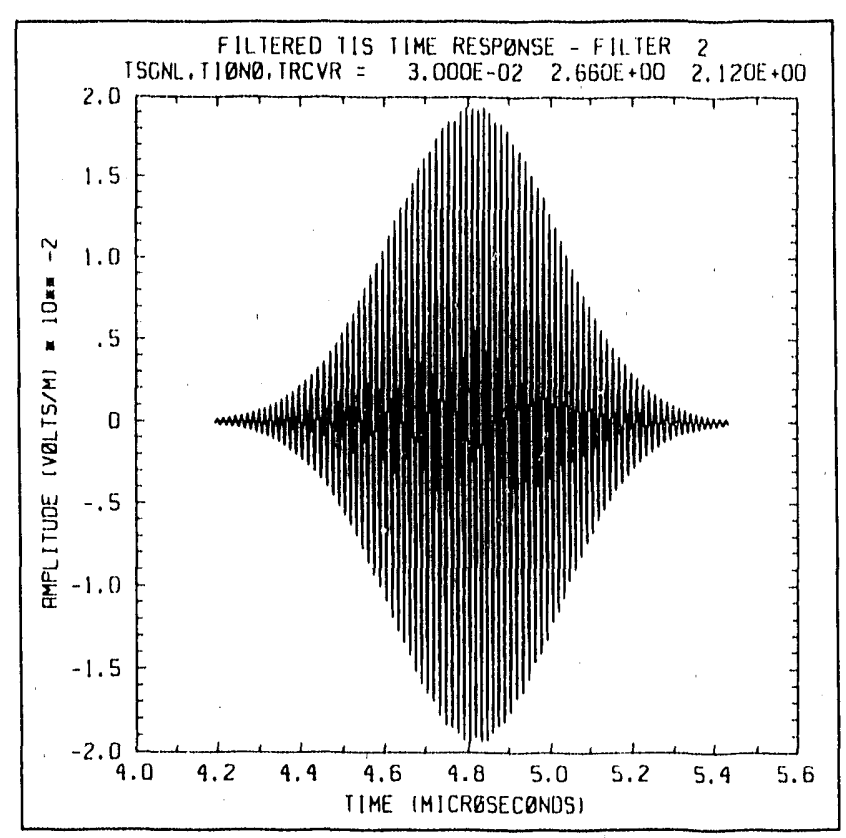

Figure 13b

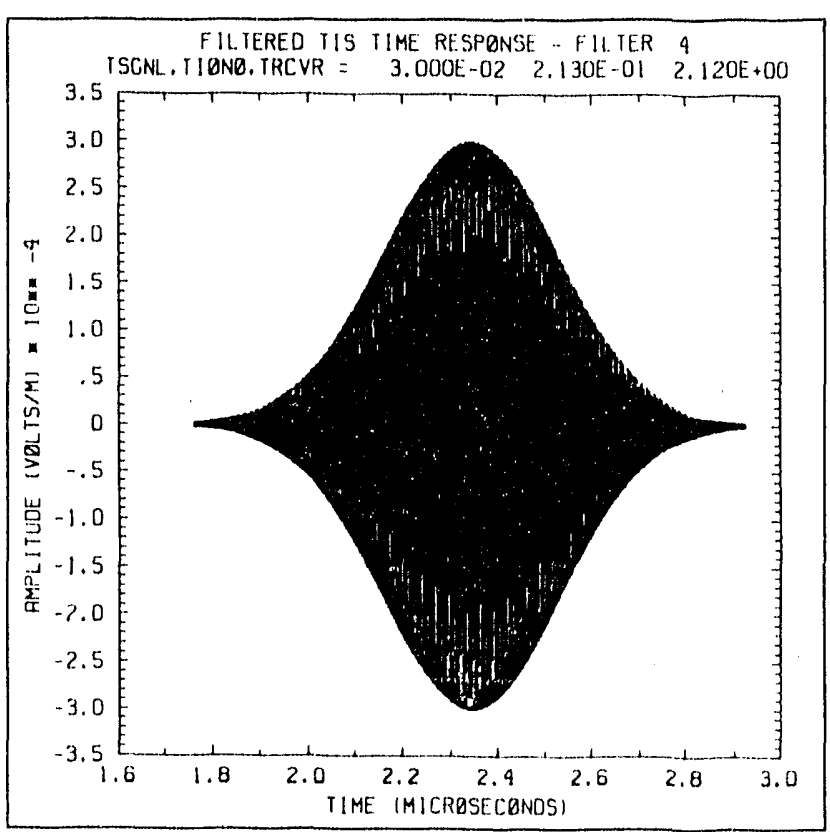

Figure 13d

Figures 13a-d (a)-(d) Filtered transionospheric signals obtained for the default super-Gaussian pulse with the four Gaussian filters shown in Figures 5 a-d, respectively. $\triangle \mathrm{TEC}$ of $1 \times 10^{13} \mathrm{~cm}^{-2}, F_{6, h}=x$, and an SNR of $70 \mathrm{~dB}$ were assumed. 


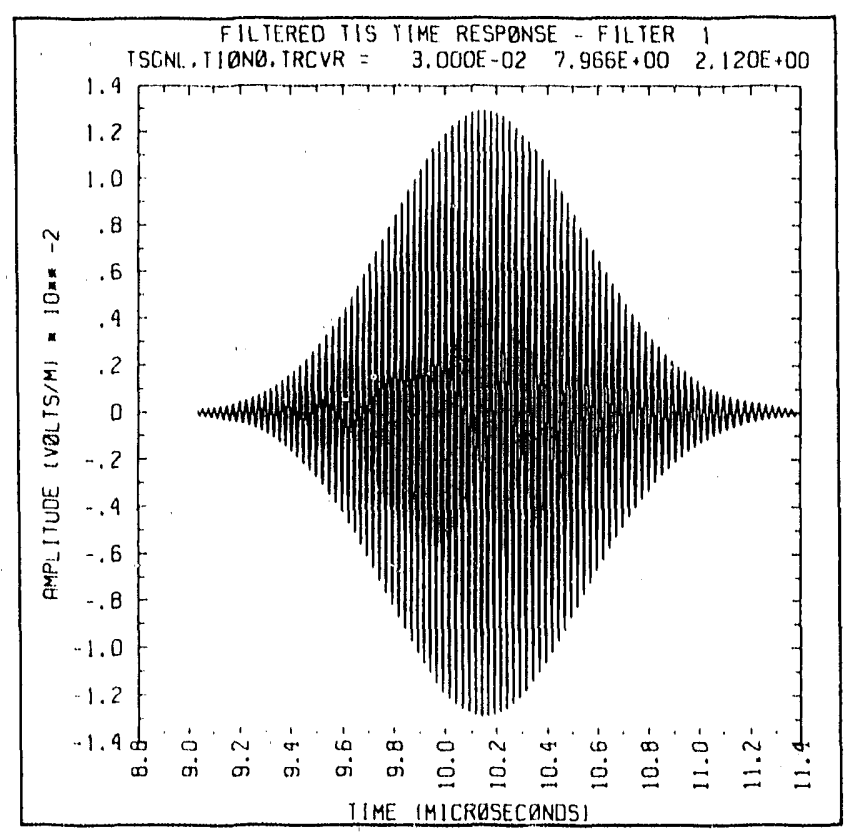

Figure 14a

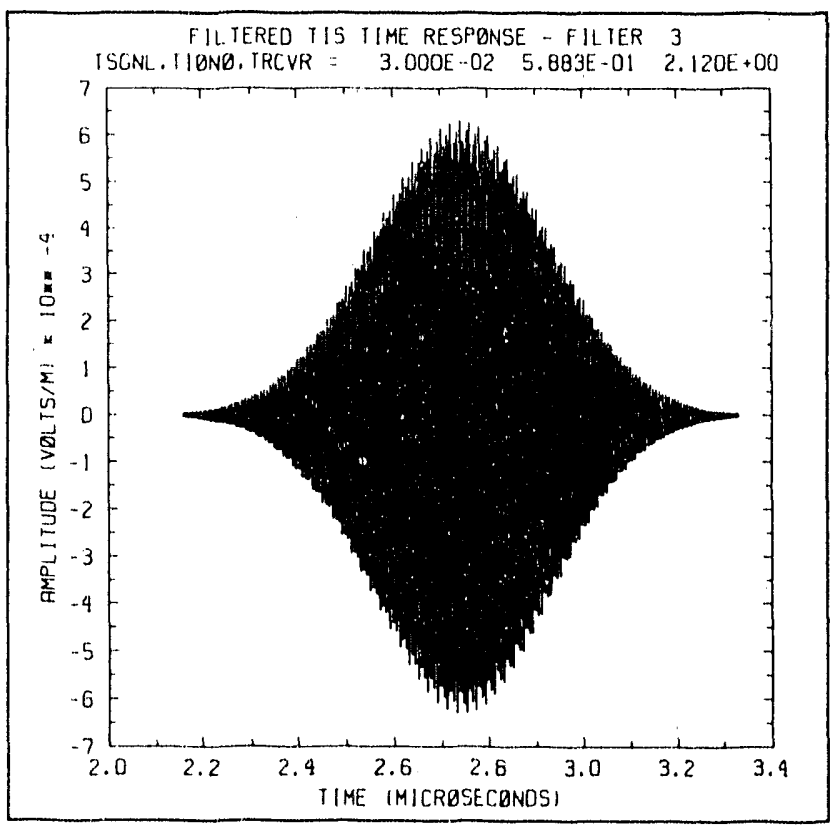

Figure 14c

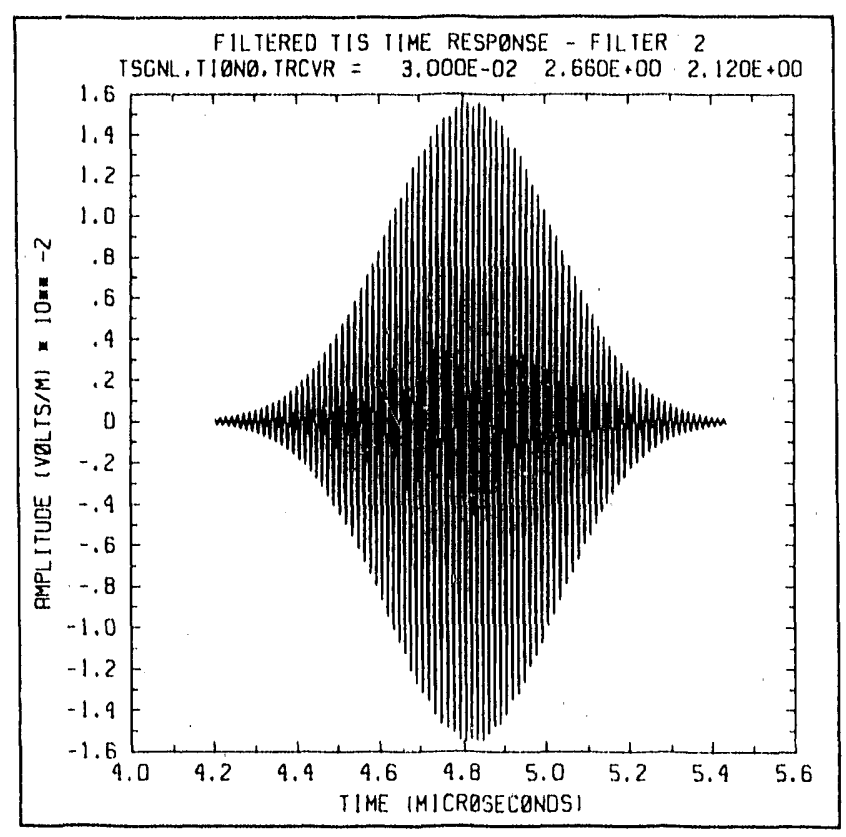

Figure 14b

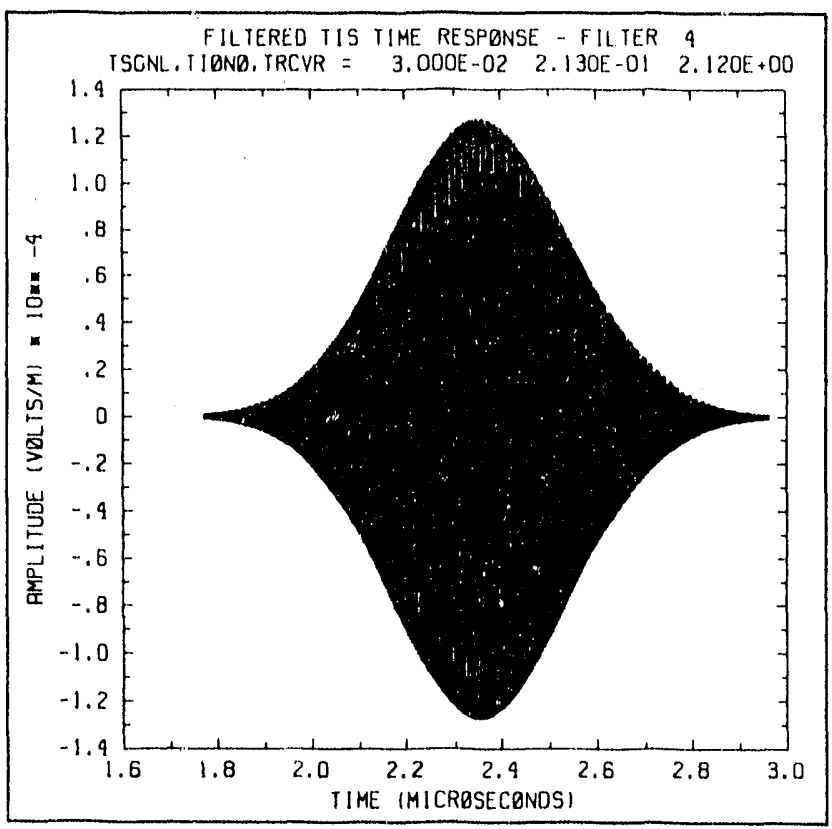

Figure 14d

Figures 14a-d (a)-(d) Filtered transionospheric signals obtained for the default super-Gaussian pulse with the four Gaussian filters shown in Figures $5 \mathrm{a}-\mathrm{d}$, respectively. A TEC of $1 \times 10^{13} \mathrm{~cm}^{-2}, F_{\text {roh }}=20 \mathrm{MHz}$, and an SNR of $70 \mathrm{~dB}$ were assumed. 


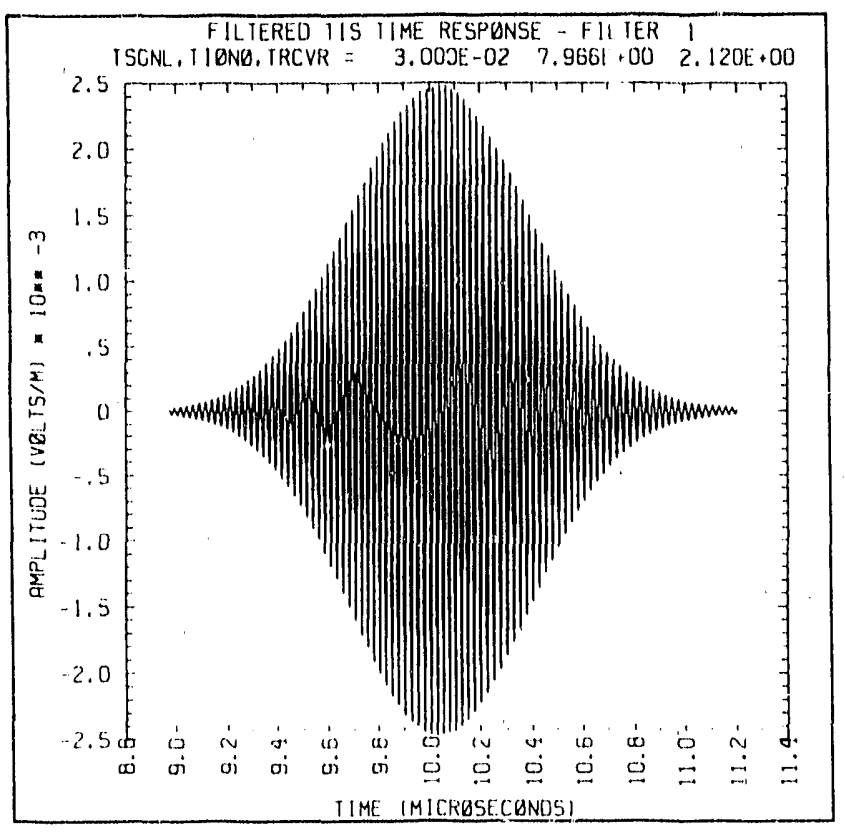

Figure 15a

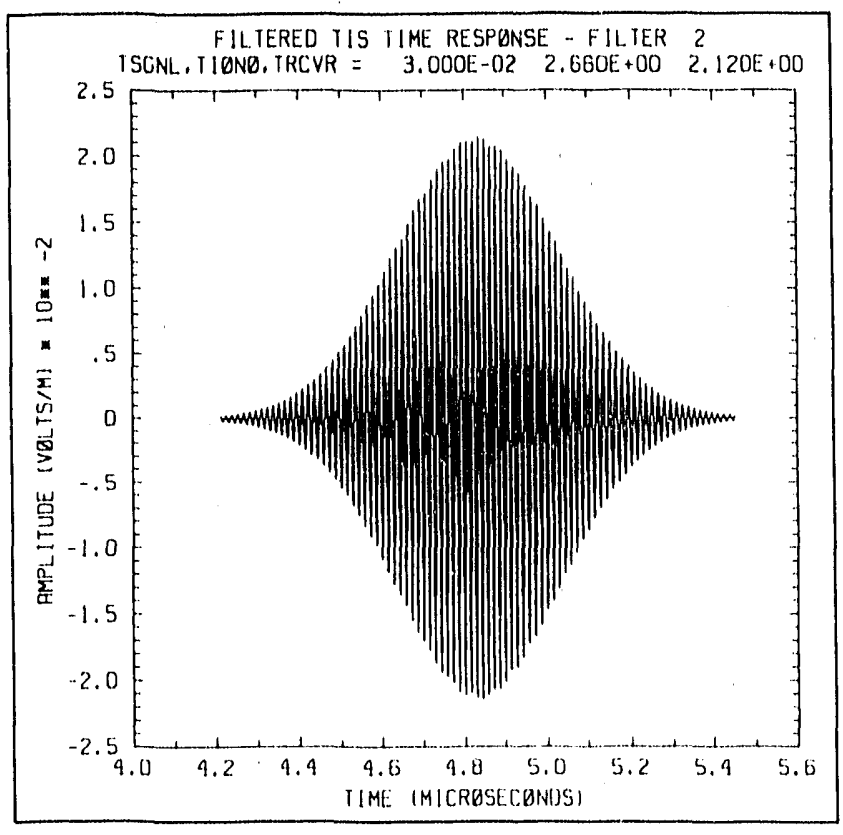

Figure 15b

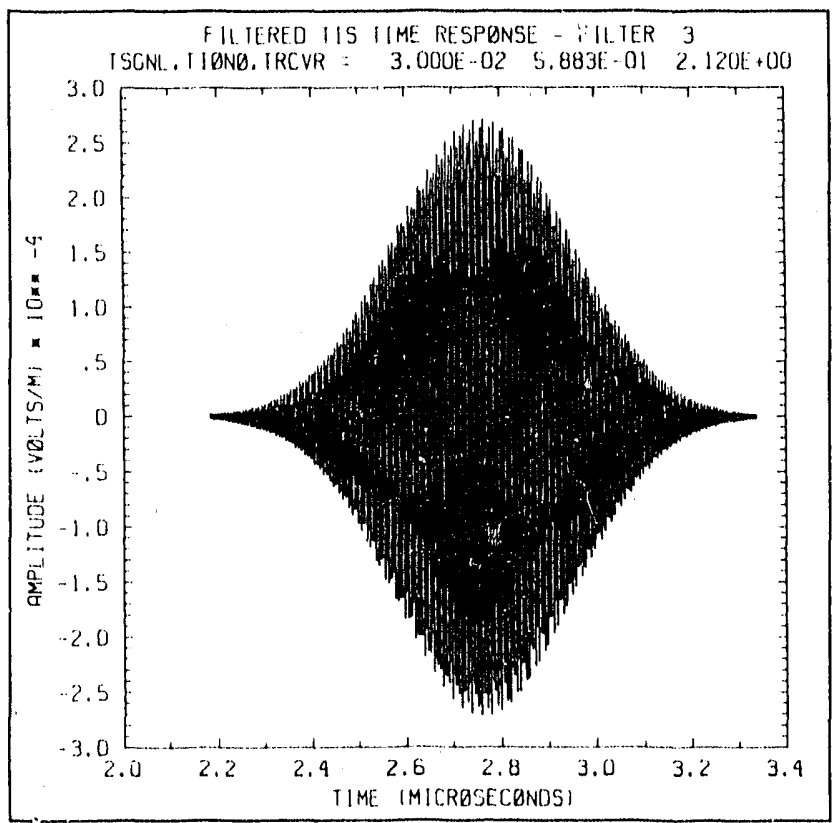

Figure 15c

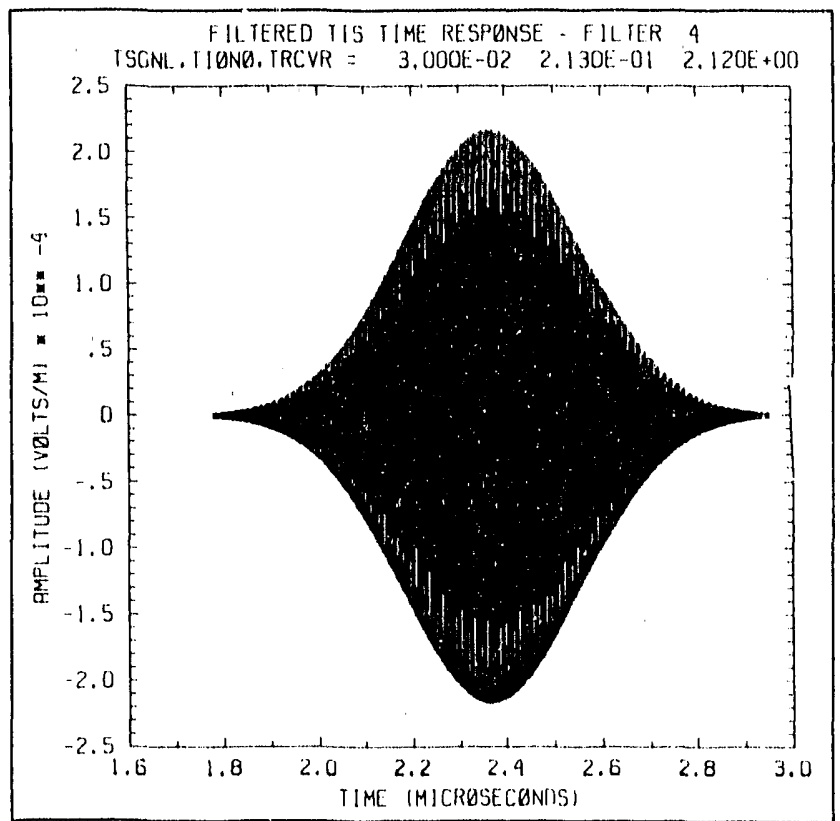

Figure 15d

Figures 15a-d (a)-(d) Filtered transionospheric signals obtained for the default super-(iaussian pulse with the four Gatussian filters shown in Figures 5a-d, respectively. A TEC of $1 \times 10^{13} \mathrm{~cm}^{2}, F_{c, w h}=10 \mathrm{MH}$, and an SNR of 70 dB were assumed. 


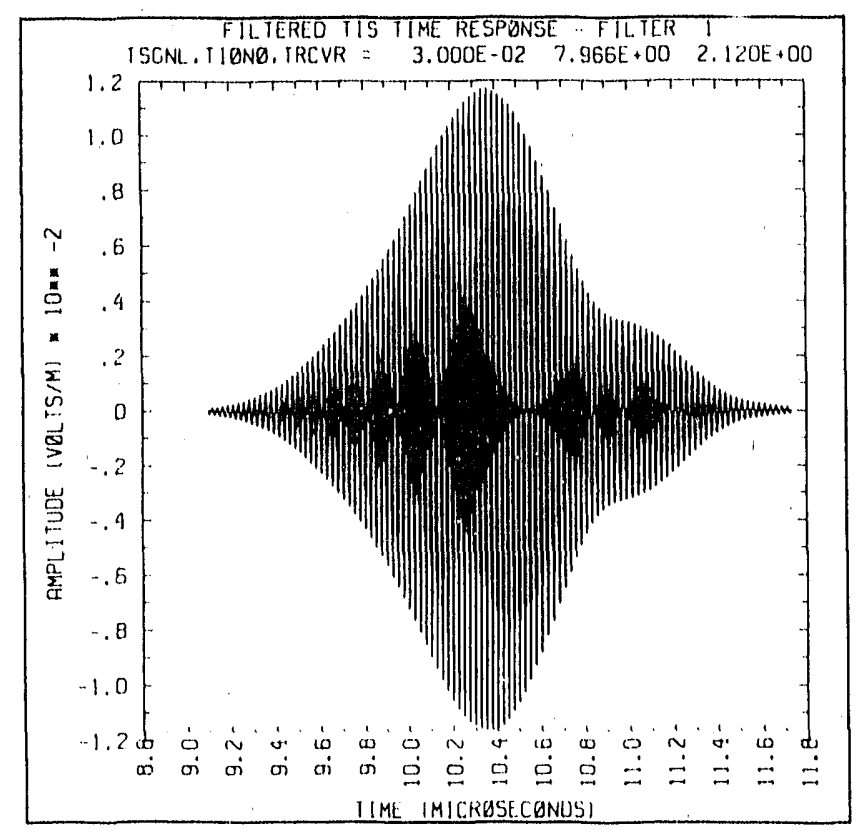

Figure 16a

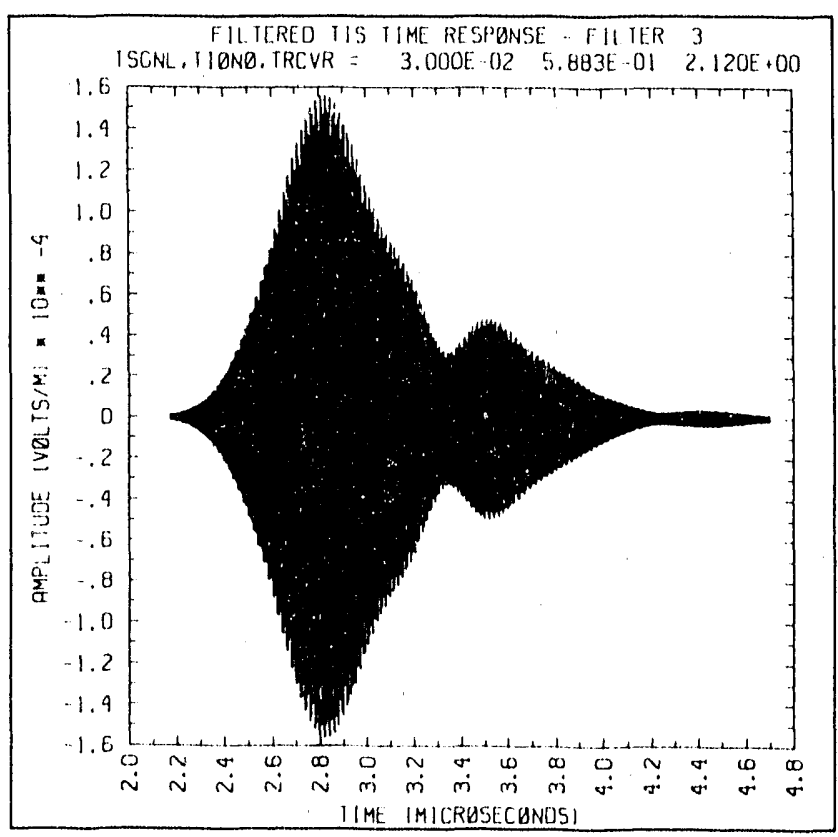

Figure 16c

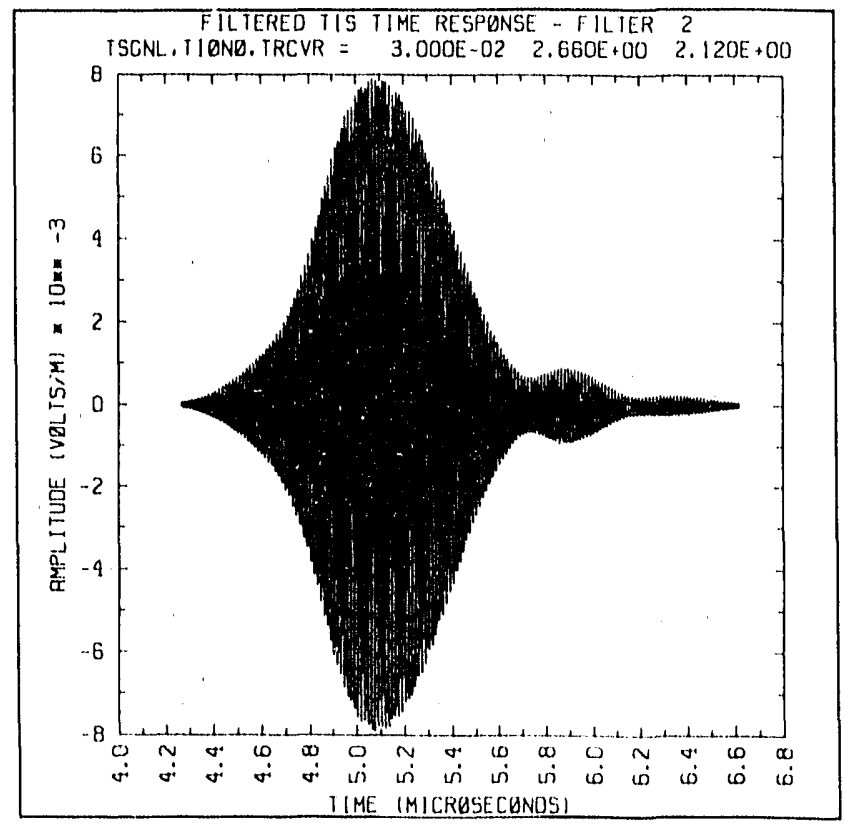

Figure 16b

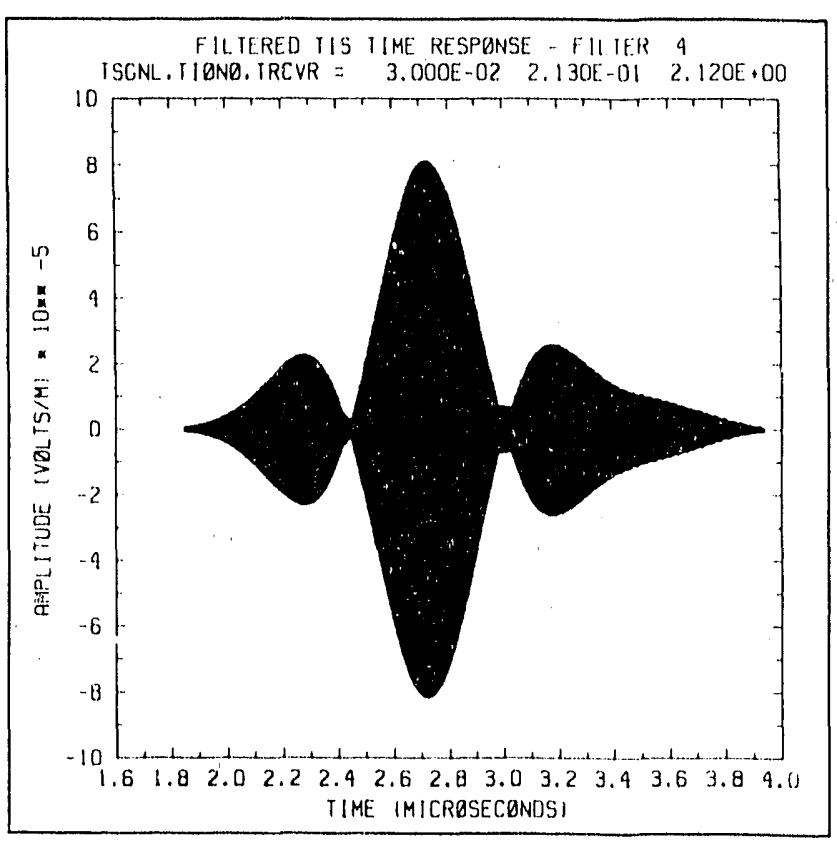

Figure 16d

Figures 16a-d (a)-(d) Filtered transionospheric signals obtained for the default super-(iaussian pulse with the four Gaussian filters shown in Figures Sa-d, respectively. A TLC of $1 \times 10^{1: 3} \mathrm{~cm}^{2}, \mathrm{~F}_{\mathrm{rah}}^{\prime}=1 \mathrm{MH} \%$, and an SNR of 70 dB wire assumed. 


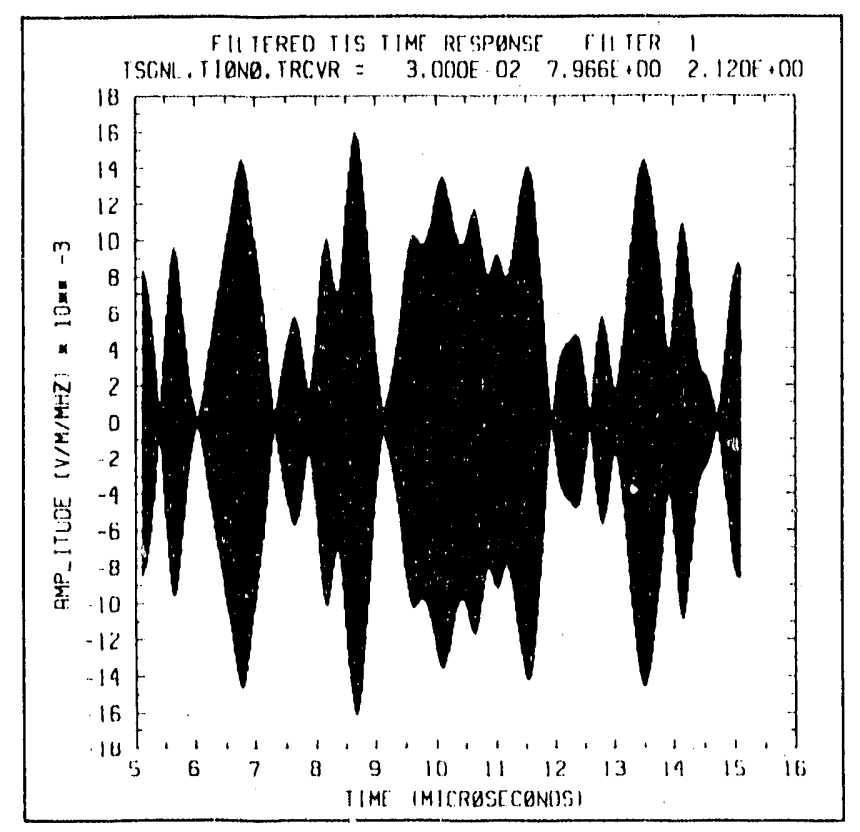

Figure 17a

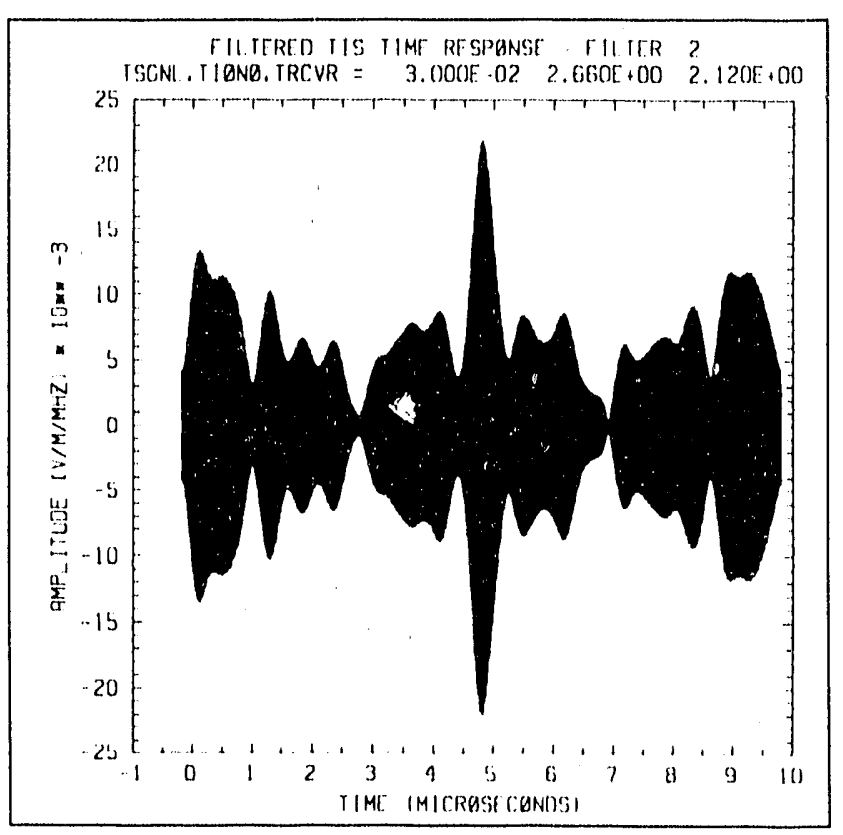

Figure 17b

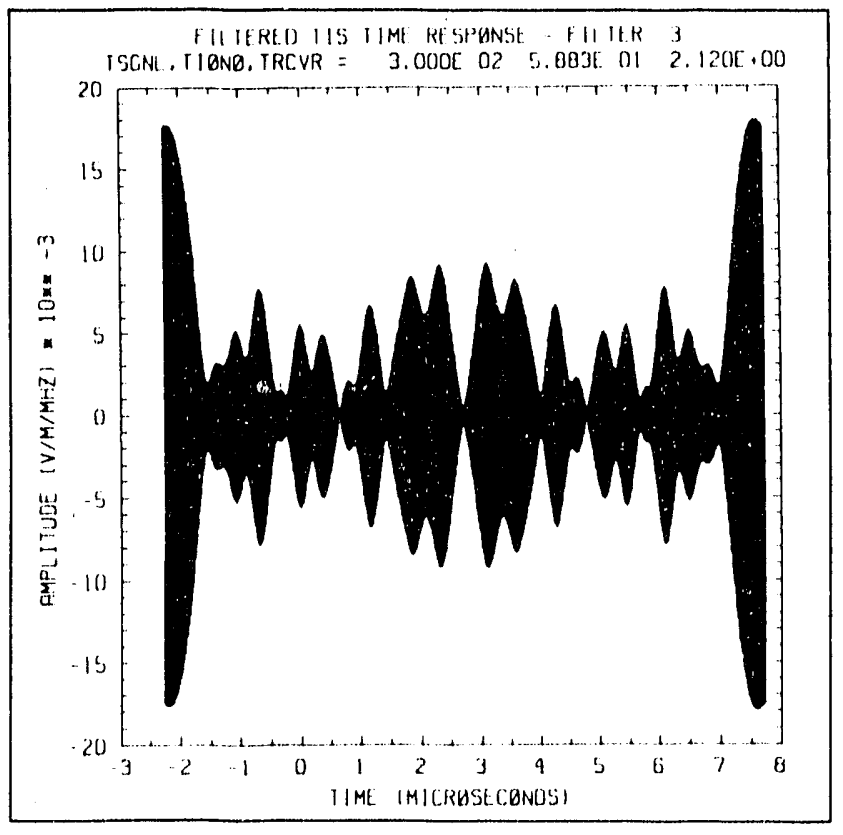

Figure $17 \mathrm{c}$

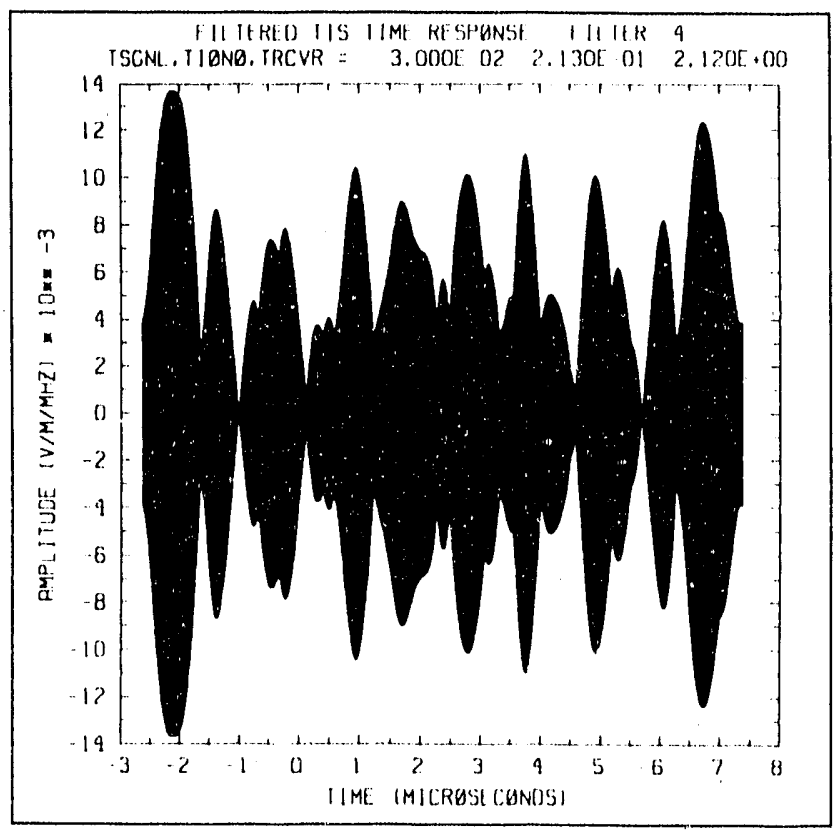

Figure 17d

Figures 17a-d (a)-(d) Filtered transionospheric signals obtained for the default super-Giatussian pulse with the four Gaussian filters shown in Figures 5 a d, respectively. A TISC of $1 \times 10^{13} \mathrm{~cm}^{2}$. F Foh $=x$, and an SNR of 100 were assumed. 


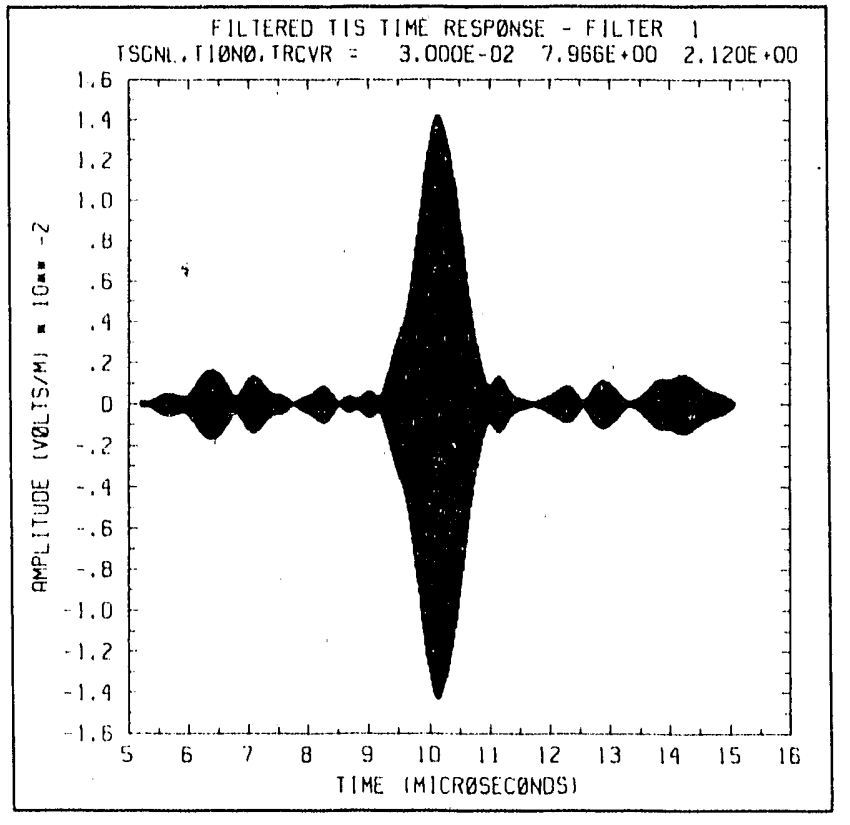

Figure 18a

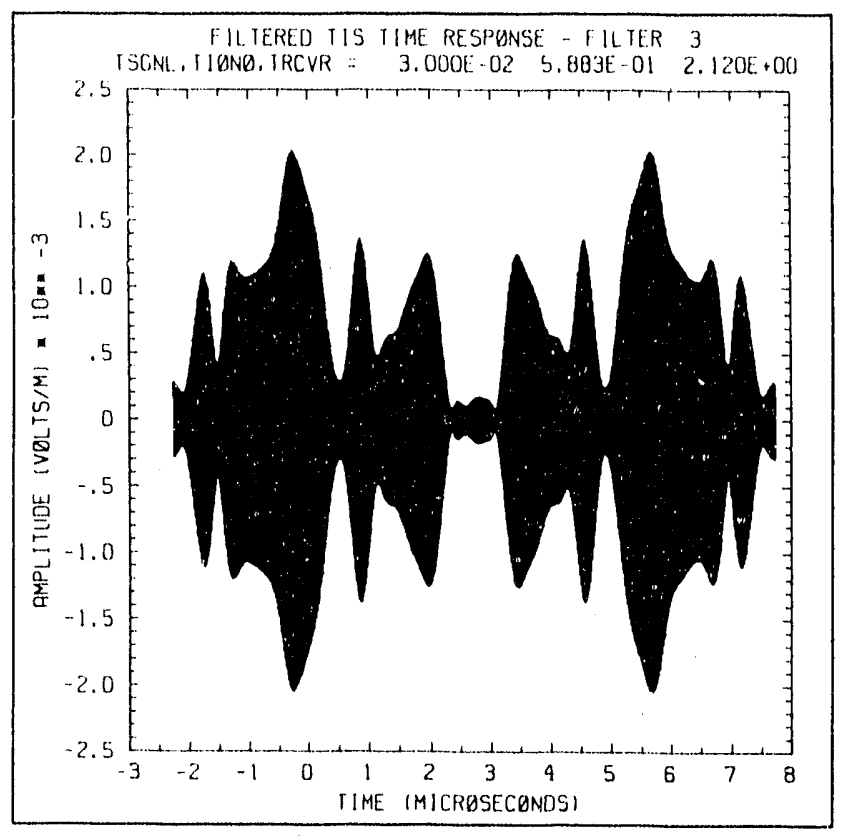

Figure 18c

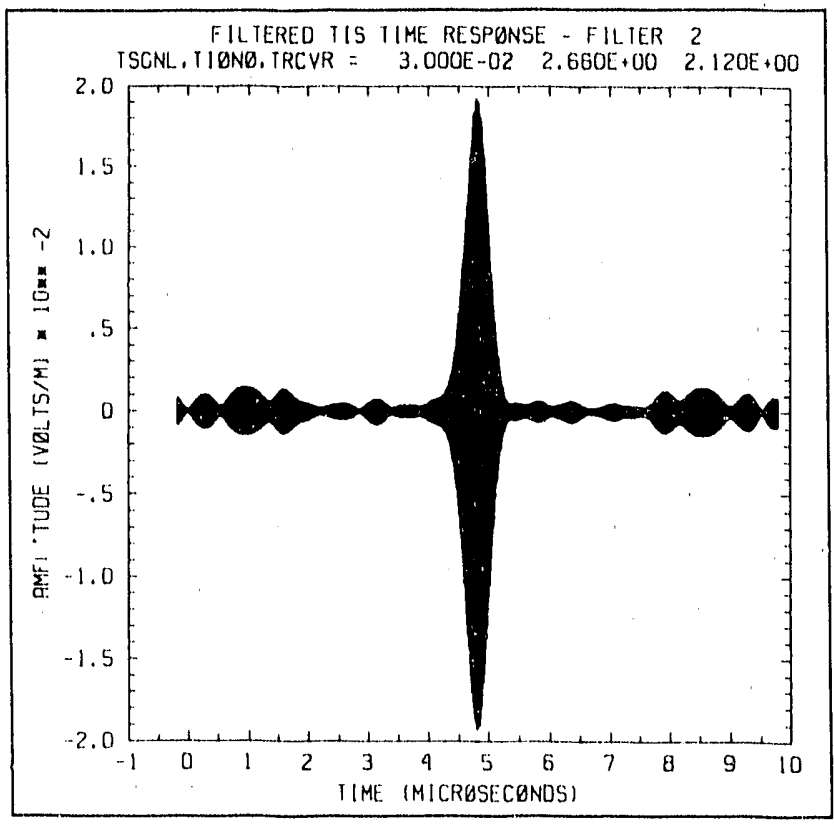

Figure 18b

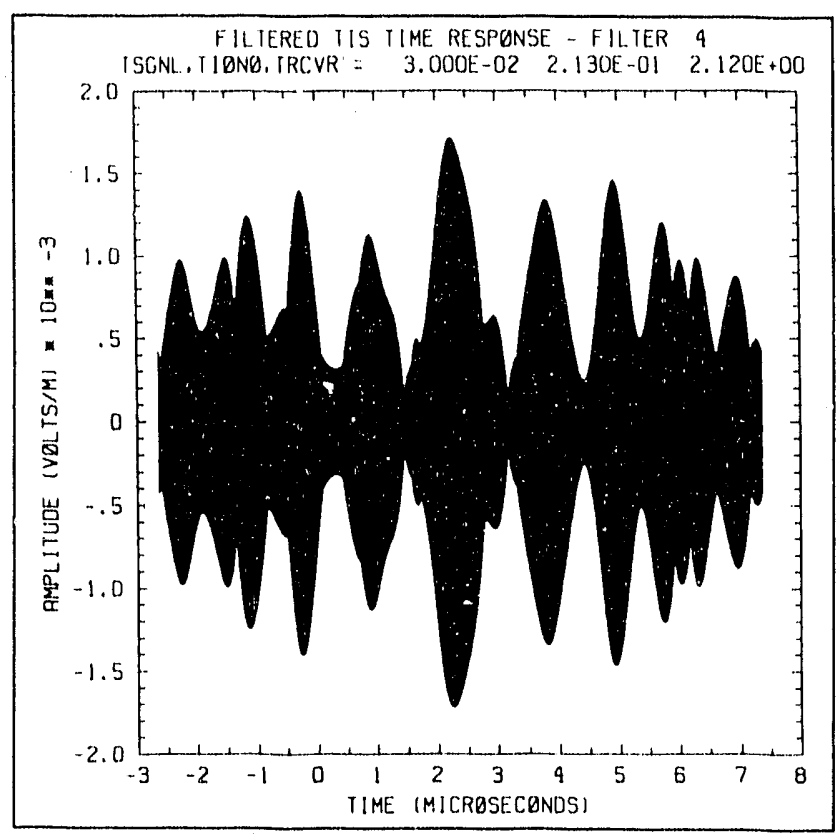

Figure 18d

Figures 18a-d (a)-(d) Filtered transionospheric signals obtained for the default super-Gaussian pulse with the four Gatussian filters shown in Figures 5 a-d, respectively. A TEC of $1 \times 10^{13} \mathrm{~cm}^{2}, F_{c, h}=\infty$, and an SNR of 10,000 were atssumed. 


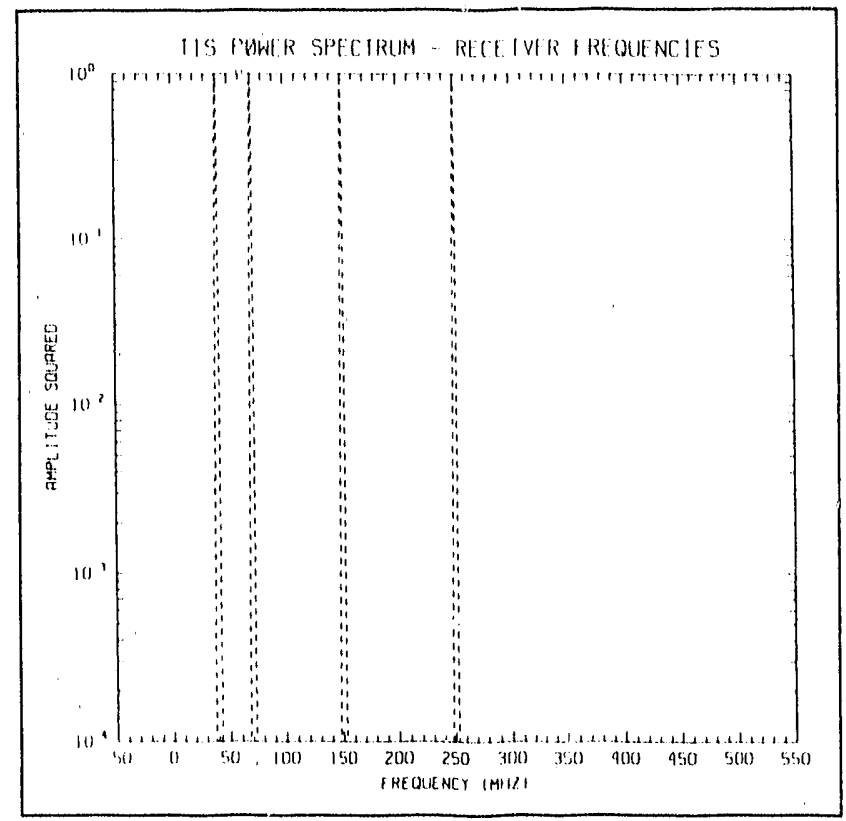

Figure 19a

Figure 19b

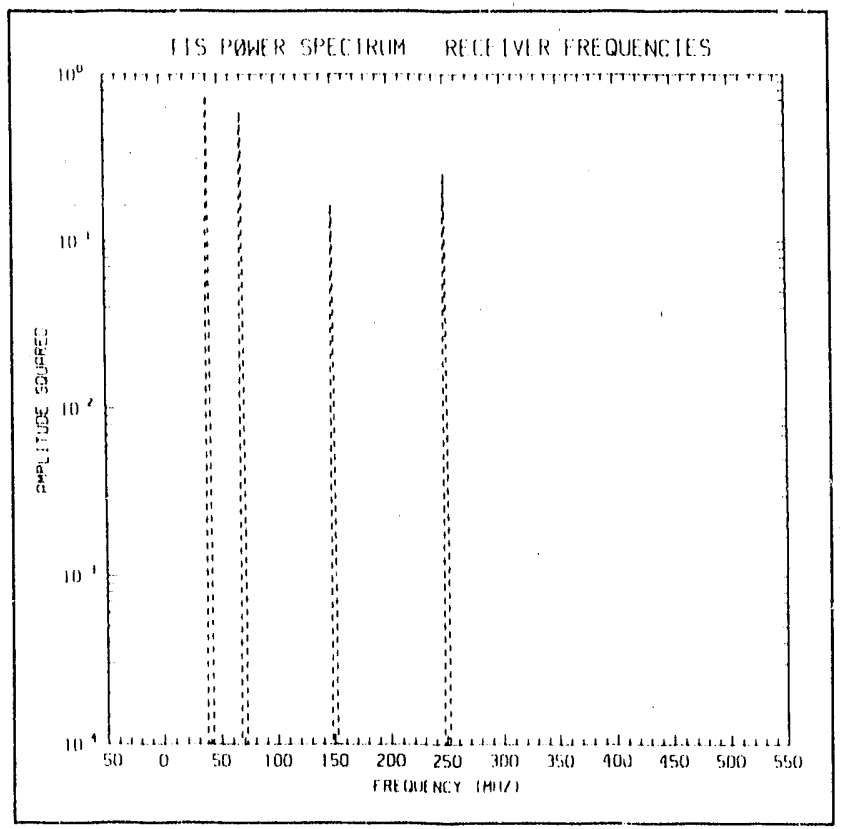

Figures 19a-f Received power in the four Gaussian filters shown in Figures 5a-d for the default delta function. The solid curve is a plot of the FFT of the input pulse while the peak values of the dashed curves represent the magnitude of the received power in the respective channels. (a) Results for a TEC. of $1 \times 10^{13} \mathrm{~cm}^{-2}, F_{c o h}=\infty$, and SNR of $70 \mathrm{~dB}$. (b)-(d) Results for a TEC of $1 \times 10^{13} \mathrm{~cm}^{-2}$, an SNR of $70 \mathrm{~dB}$, and $F_{c o h}$ of 20,10 and $1 \mathrm{MHz}$, respectively, (e)-(f) Results for a TEC of $1 \times 10^{13} \mathrm{~cm}^{-2}, F_{c o h}^{\prime}=\infty$, and SNR of 100 and $10,(0)($, respectively. 


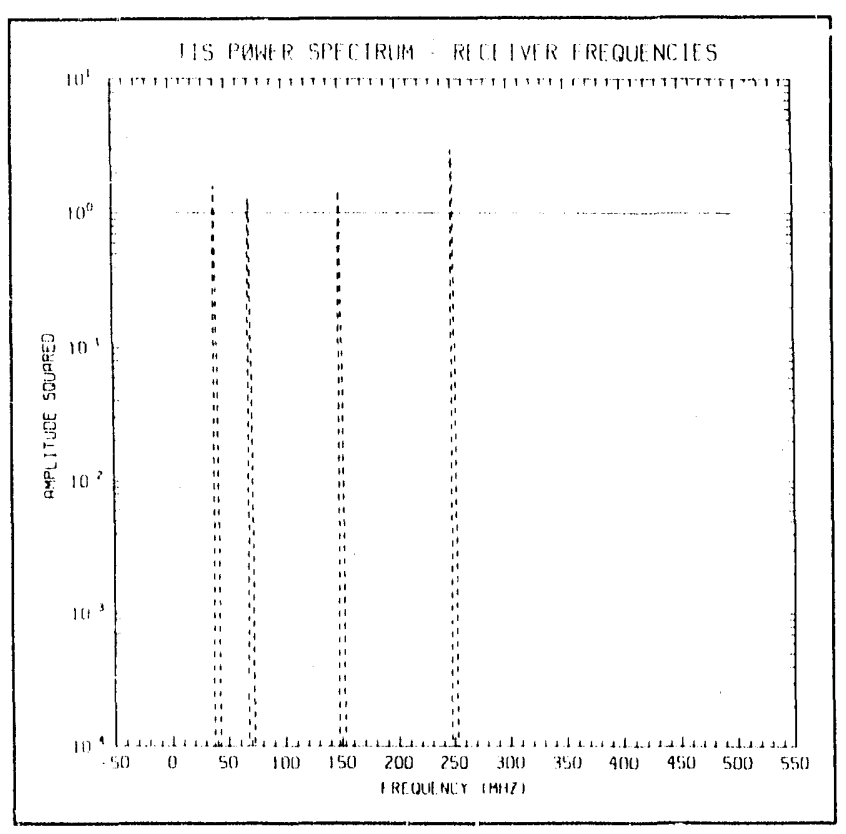

Figure 19c

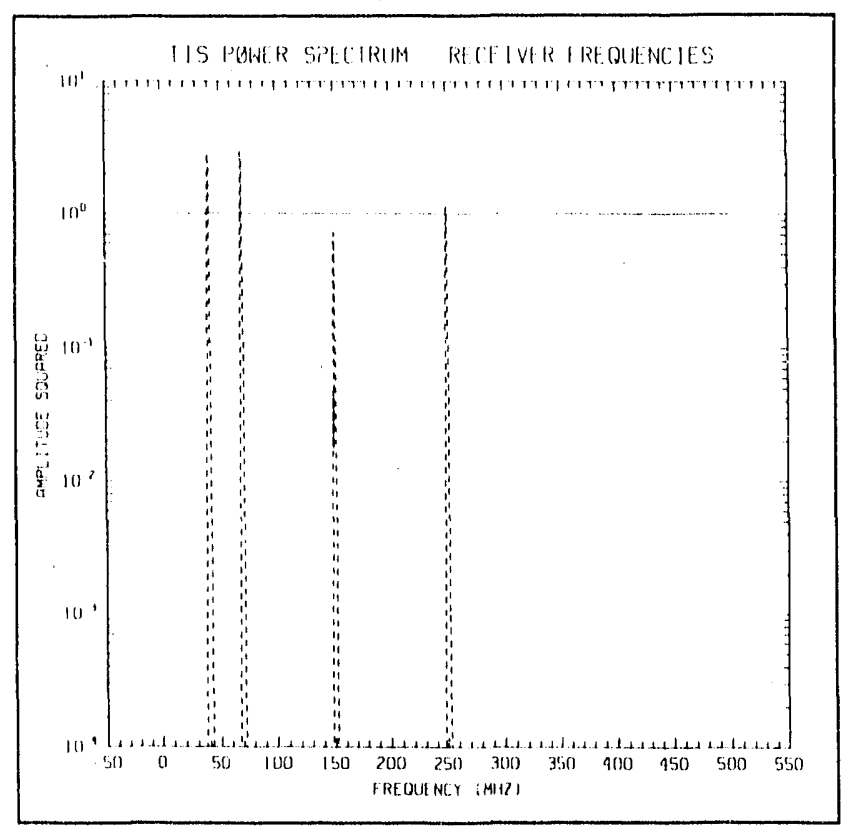

Figure 19d

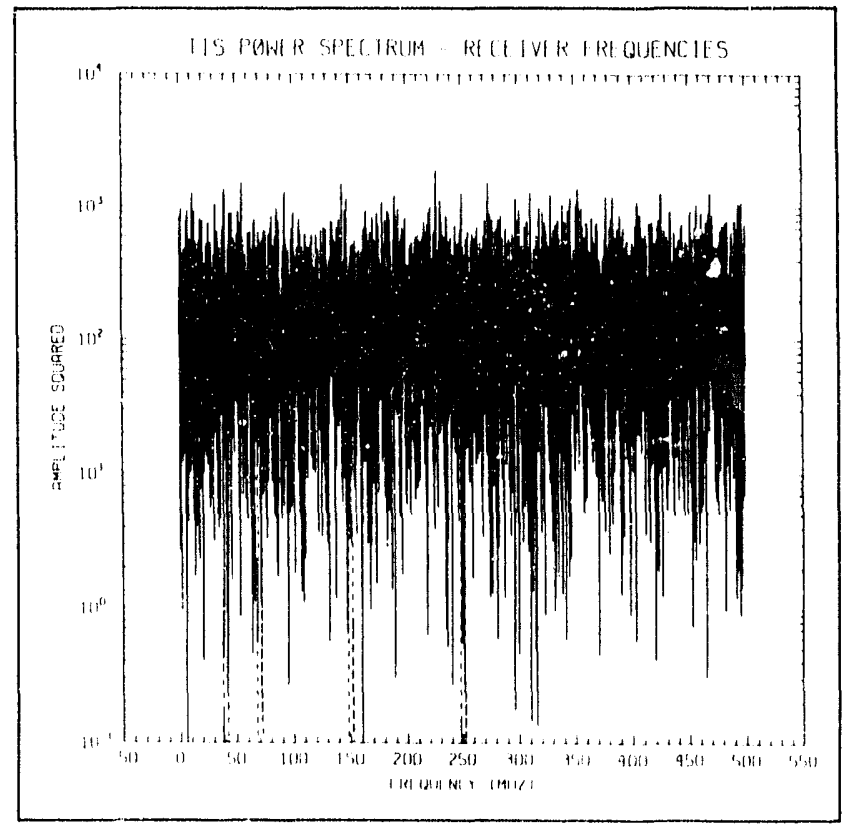

Figure 19e

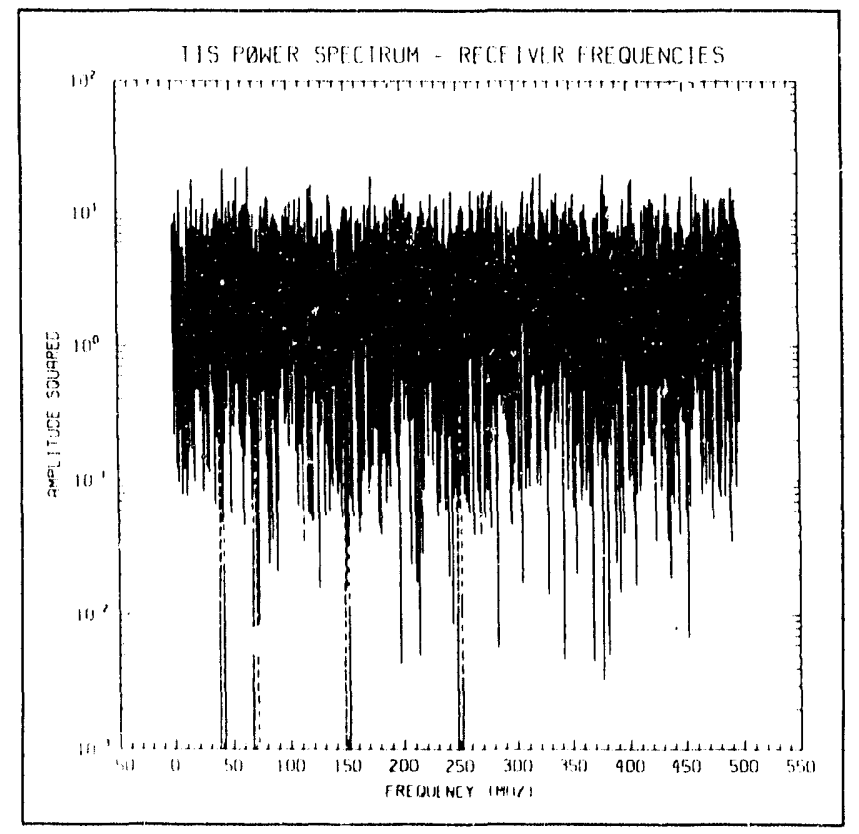

Figure $19 f$ 


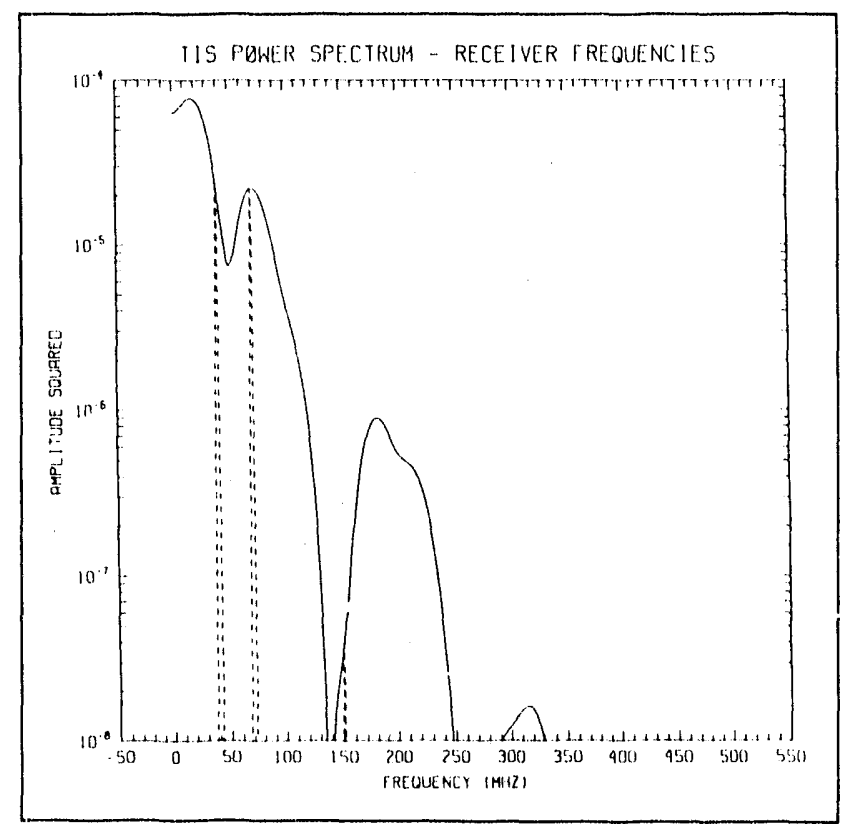

Figure 20a

Figure 20b

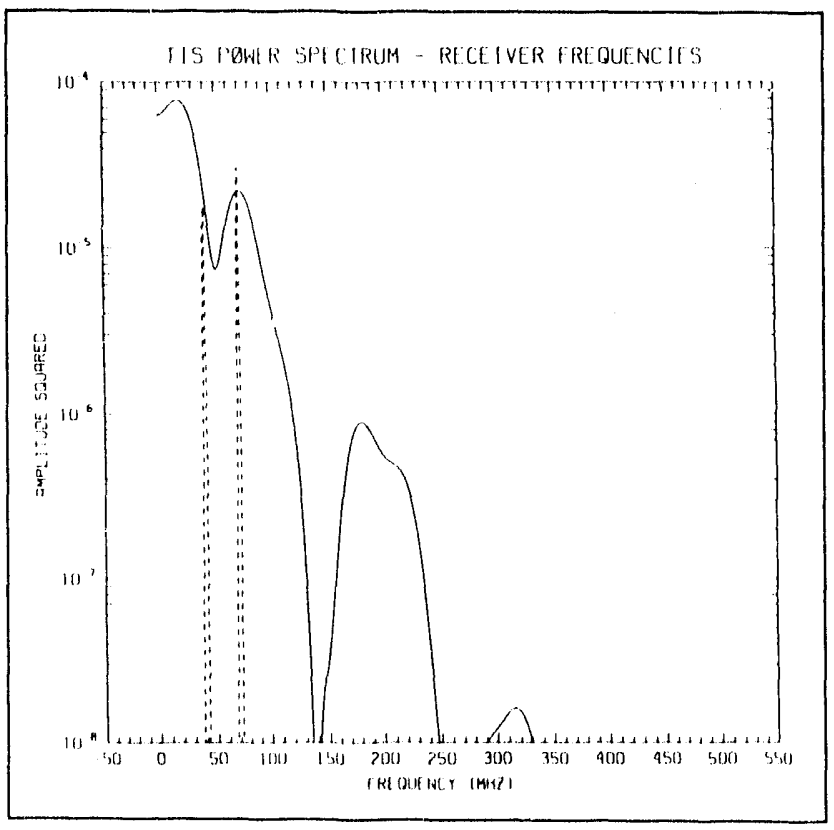

Figures 20a-f Received power in the four Gaussian filter: shown in Figures 5a-d for the default super-Gaussiant pulse. The solid curve is a plot of the FFT of the input pulse while the peak values of the dashed curves represent the magnitude of the received power in the respective channels. (a) Results for a TEC of $1 \times 10^{1: 3} \mathrm{~cm}^{-2}, F_{c, h}=x$, and SNR of $70 \mathrm{~dB}$. (b)-(d) Results for a TEC of $1 \times 10^{13} \mathrm{~cm}^{-2}$, an SNR of $70 \mathrm{~dB}$, and $F_{c o h}$ of 20,10 and $1 \mathrm{MHz}$, respectively. (e)-(f) Results for a TEC of $1 \times 10^{13} \mathrm{~cm}^{-2}, F_{6, \alpha}=x$, and SNR of 100 and $10,(0) 0$, respectively. 


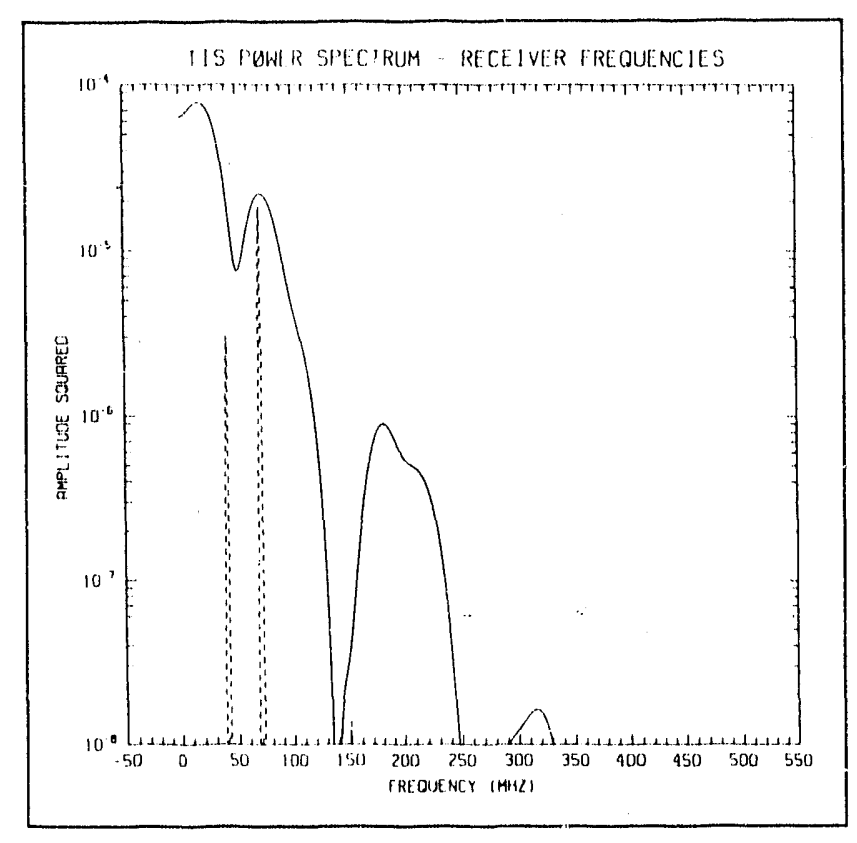

Figure 20c

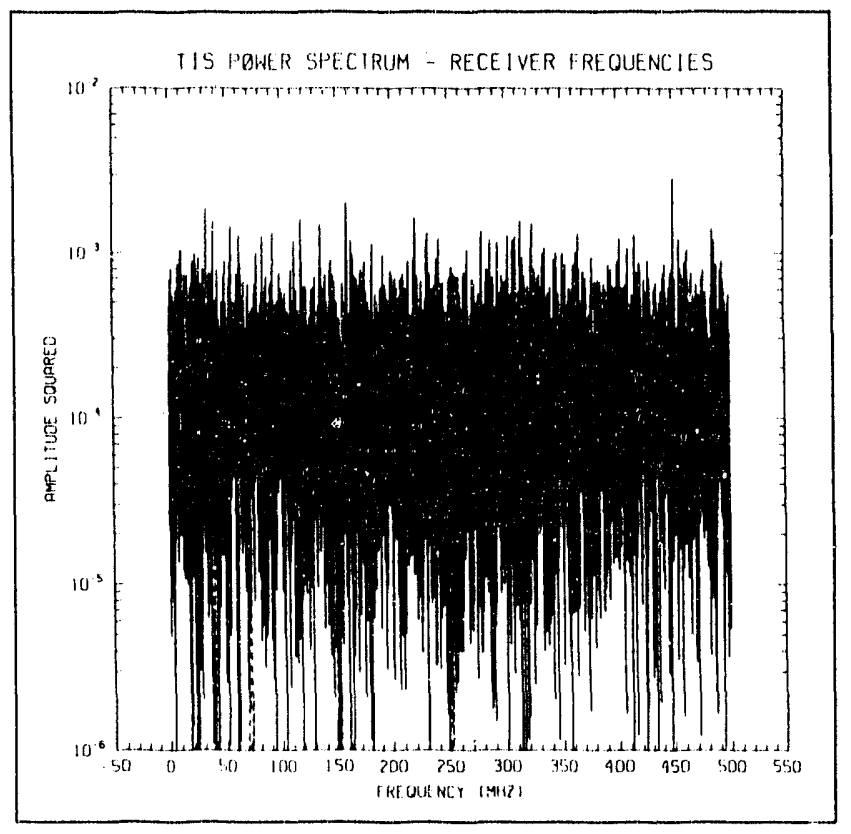

Figure 20e

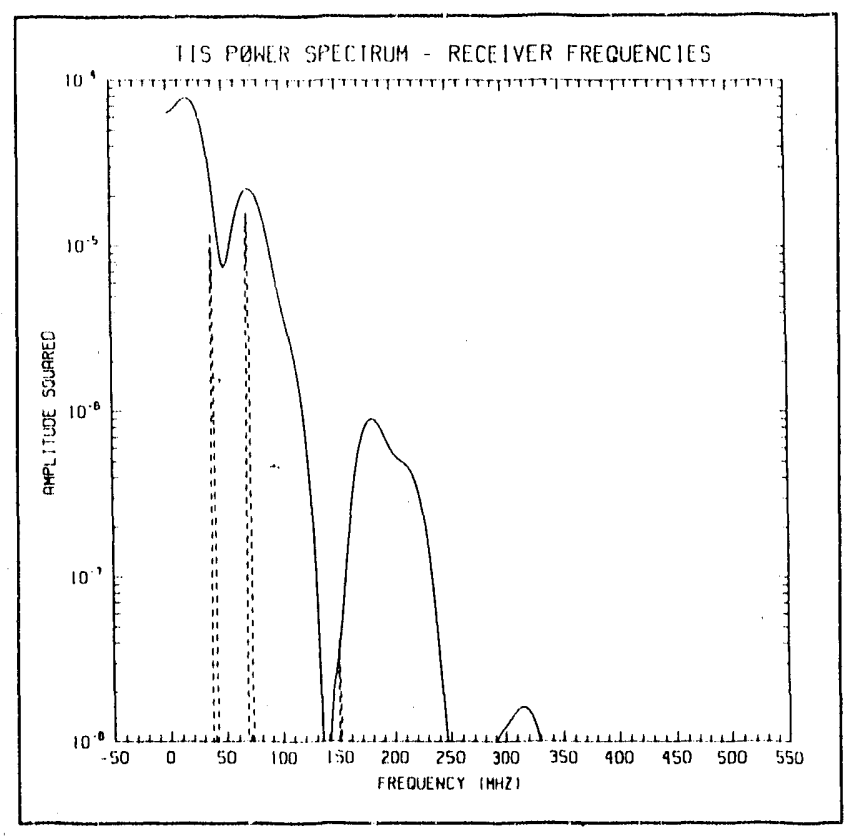

Figure 20d

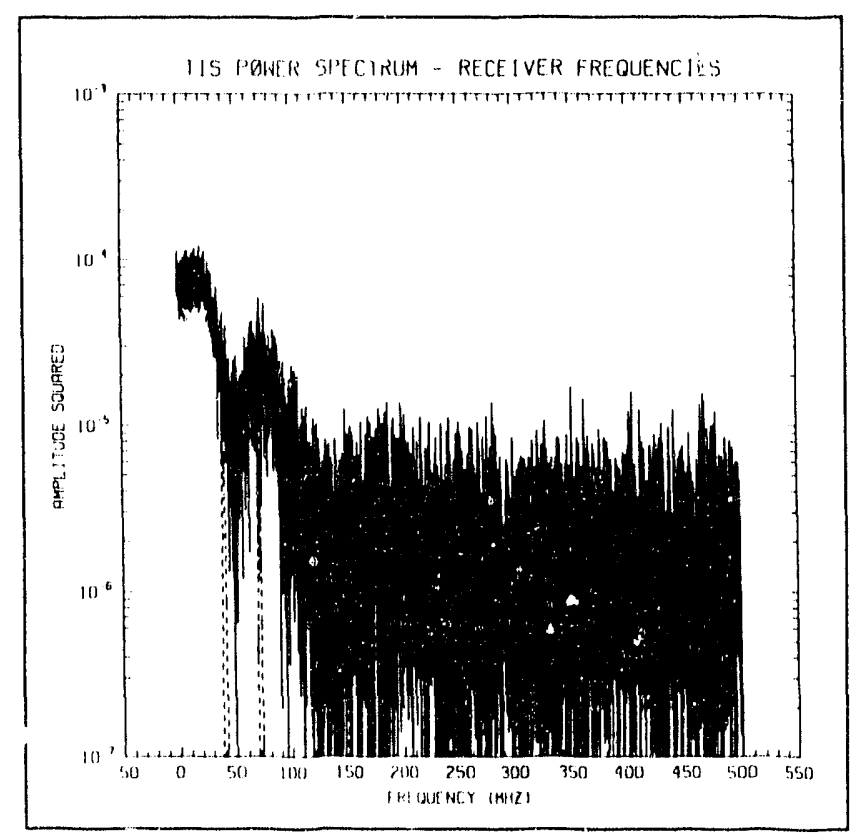

Figure 20f 


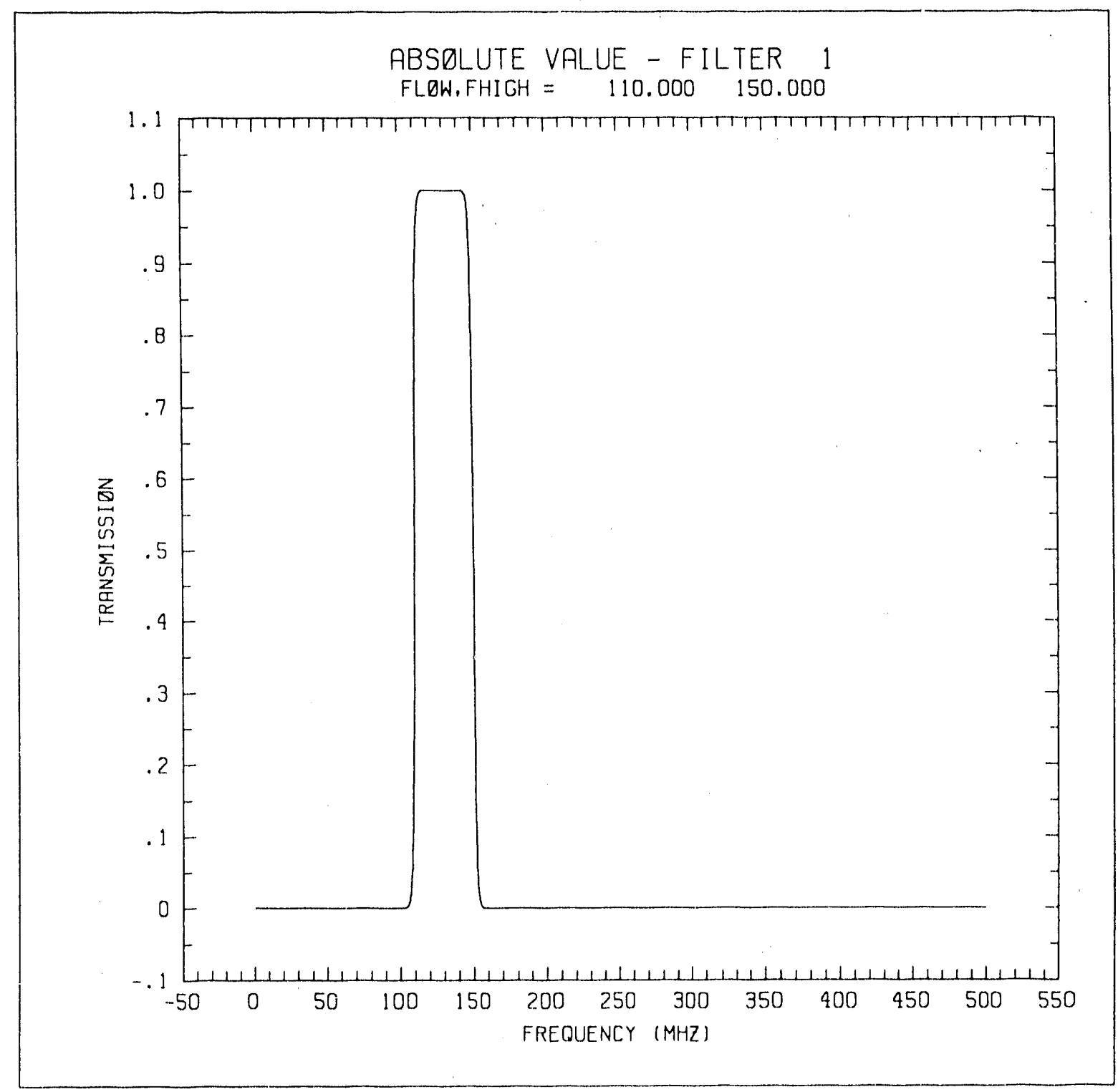

Figure 21 Transmission profile for a wideband filter with a central frequency of $130 \mathrm{MHz}$ and FWHM of 40) $\mathrm{MHz}$. 


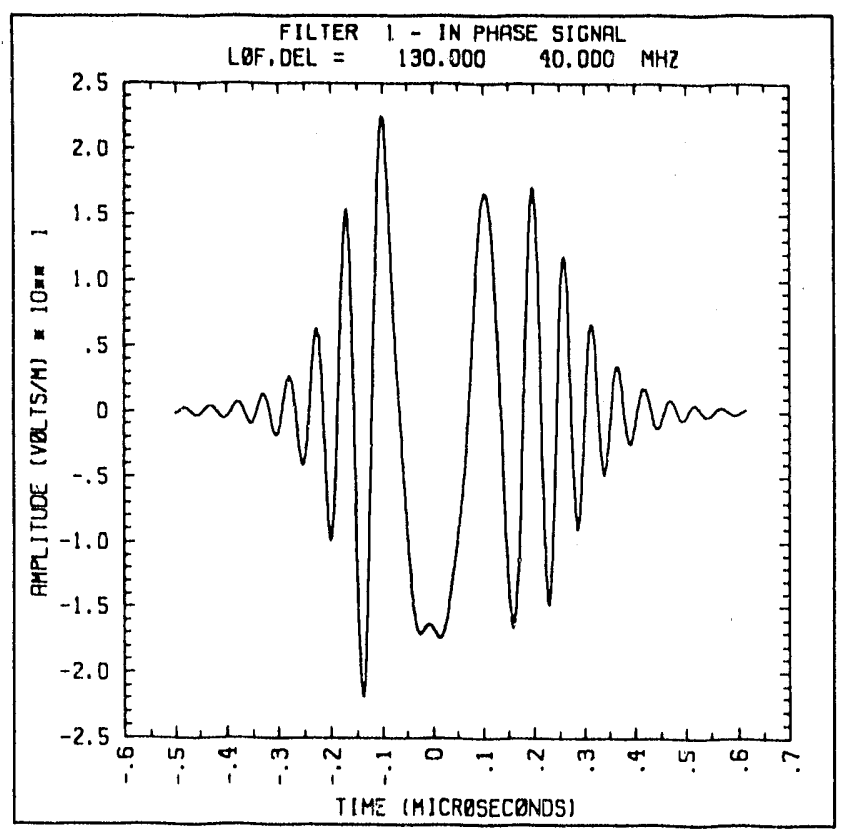

Figure 22a

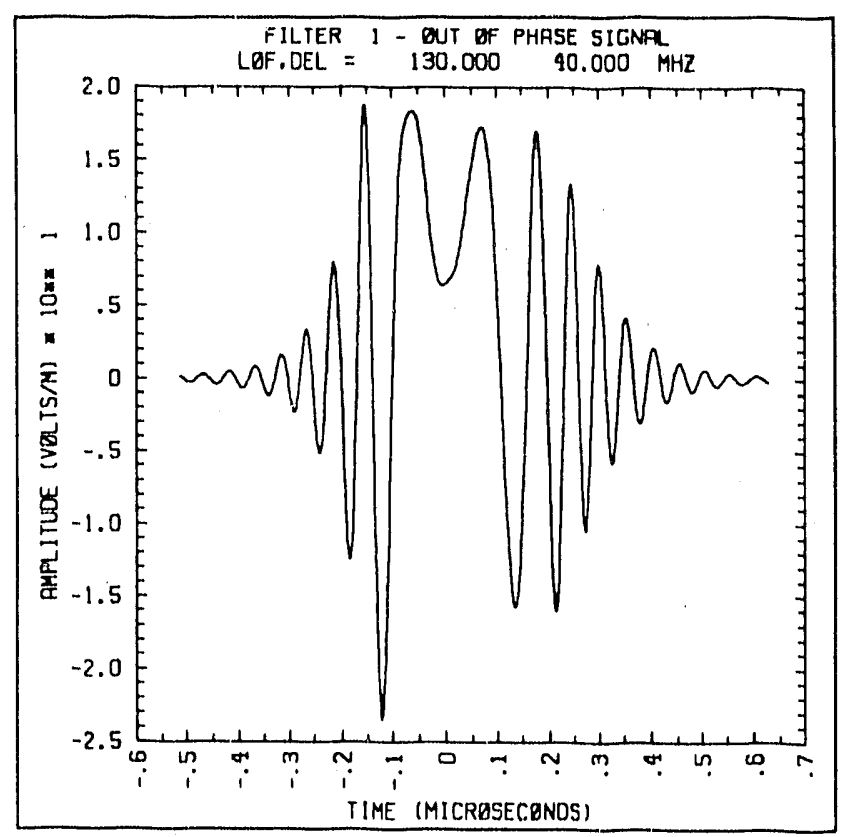

Figure 22b

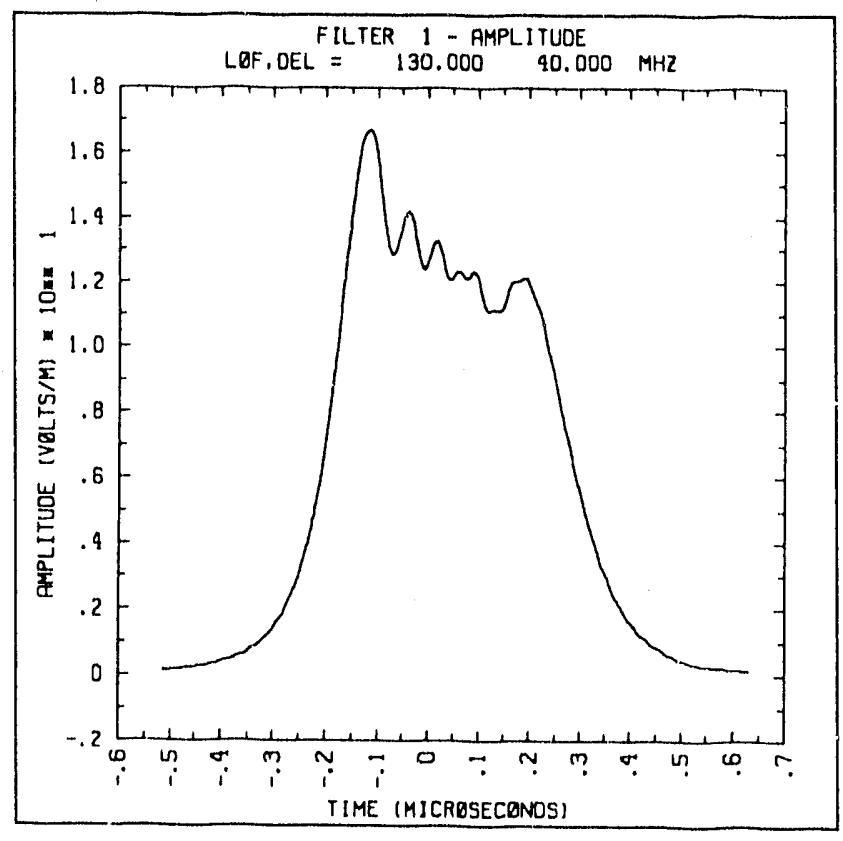

Figure 22c

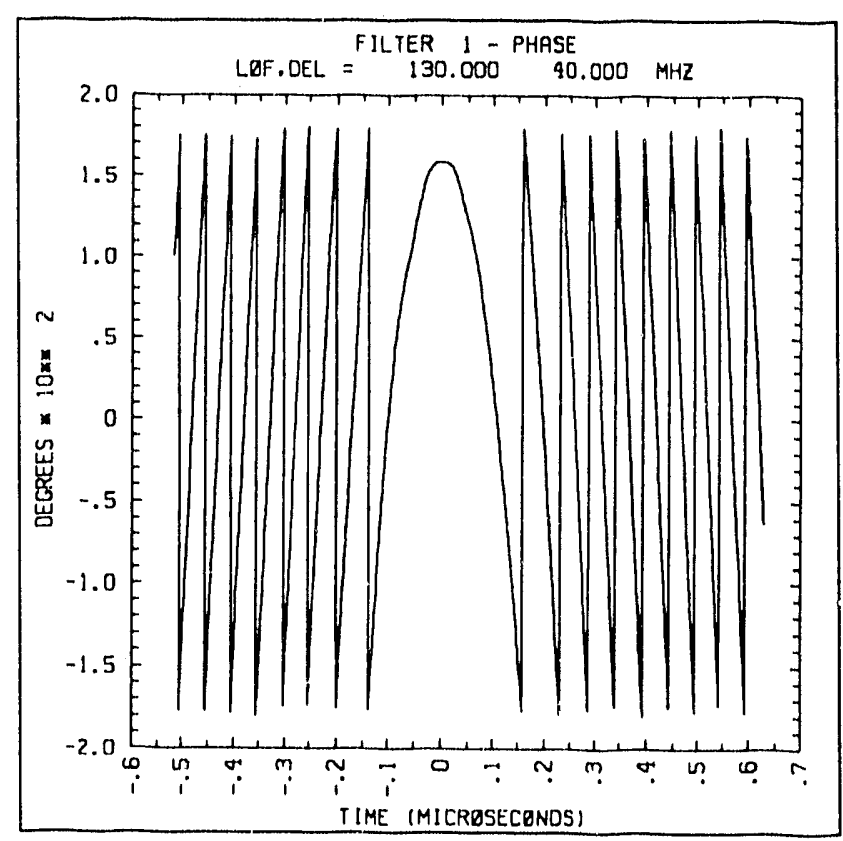

Figure 22d

Figures 22a-d Results of quadrature detection with the filter shown in Figure 21 and for the default delta function and an ionosphere with TEC $=1 \times 10^{13} \mathrm{~cm}^{-2}, F_{c o h}=\infty$, and $S N R=70 \mathrm{~dB}$. (a) In-phase component (1), (b) Out-of-phase component (Q), (c) Amplitude and, (d) Phase of filtered transionospheric signal. 


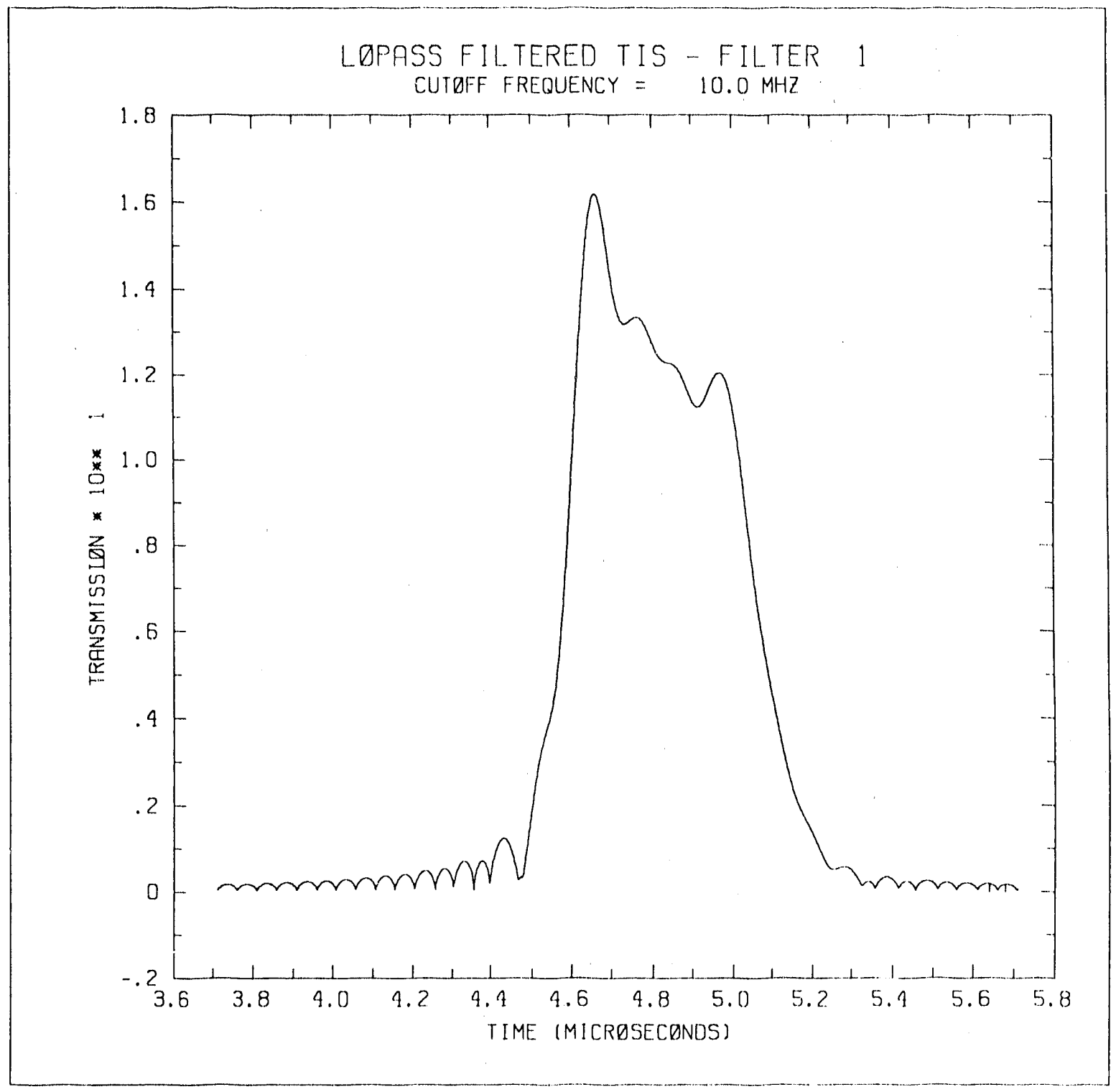

Figure 23 Results of square-law detection with the filter shown in Figure 21 and for the default delta function and an ionosphere with TEC $=1 \times 10^{13} \mathrm{~cm}^{-2}, F_{\text {coh }}=\infty$. and $\mathrm{SNR}=70 \mathrm{~dB}$. The low-pass filter used in this exercise has a FWHM equal to $10 \mathrm{MHz}$. 


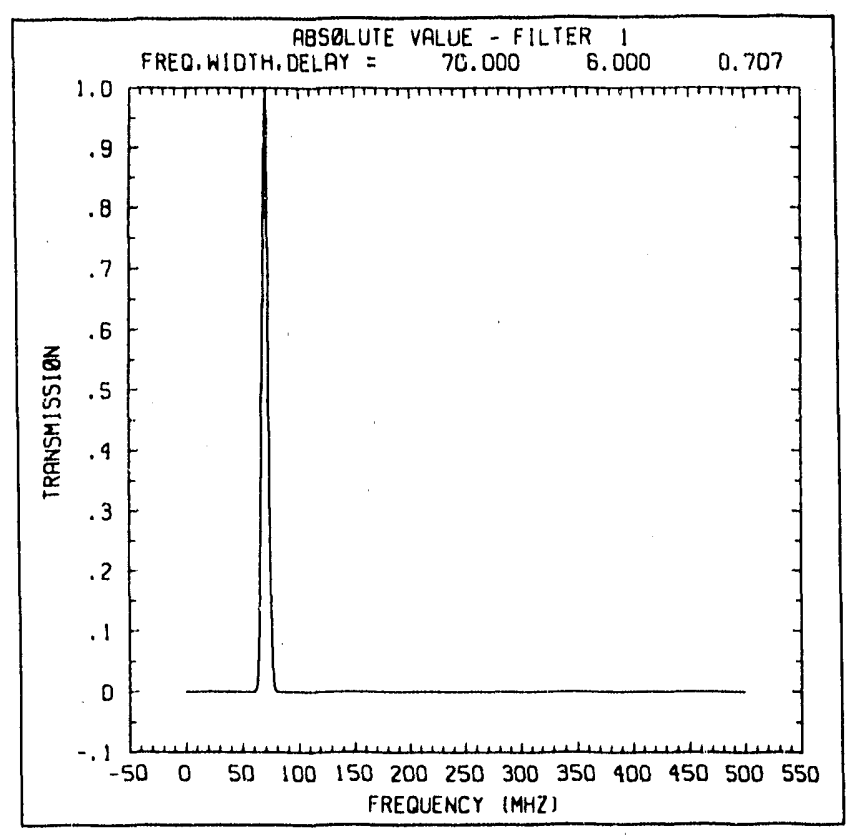

Figure 24a

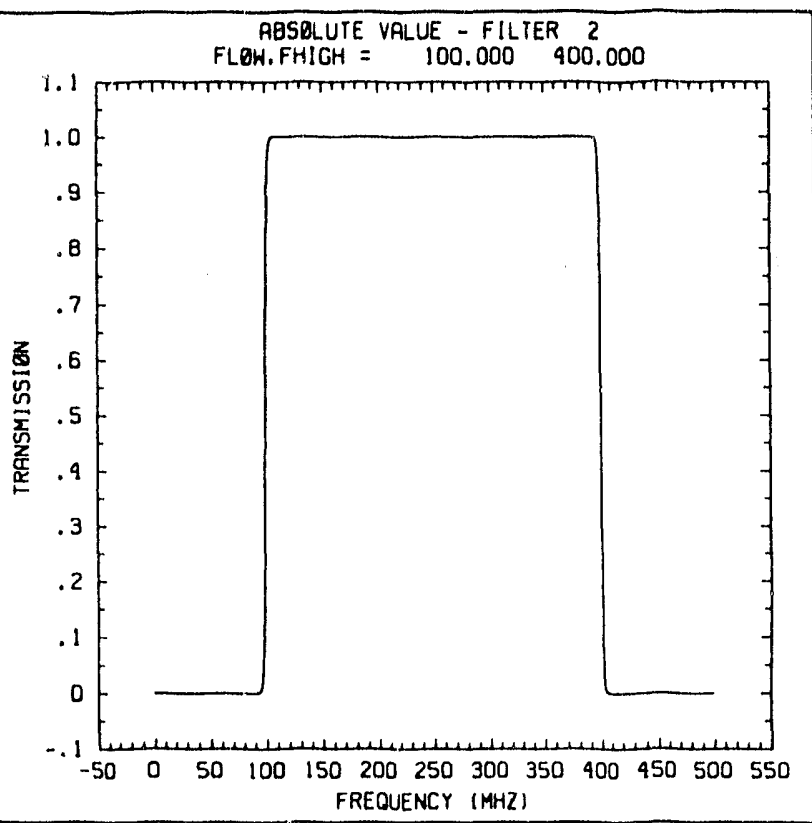

Figures 24a-b Filter transmission functions used in TOA analysis. (a) Wideband filter with a FWHM of $3(0) \mathrm{MHz}$ and $50 \%$ transmission points at 100 and $400 \mathrm{MHz}$ (b) Narrowband Gaussian filter with a central frequency of $70 \mathrm{MHz}$ and FWHM of $6 \mathrm{MHz}$. 


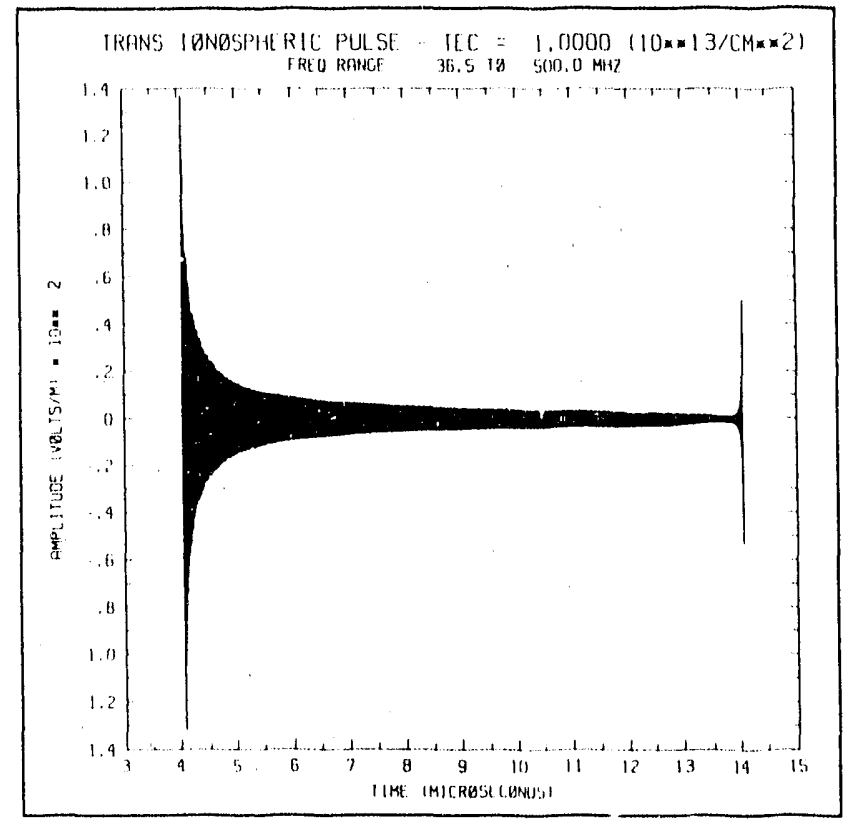

Figure 25a

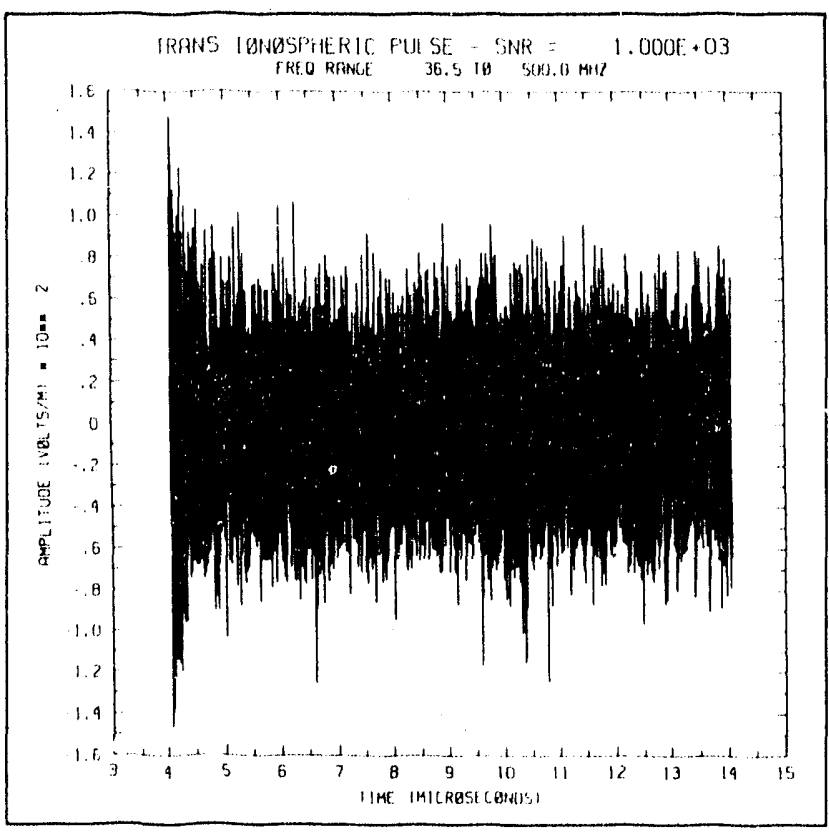

Figure 25b

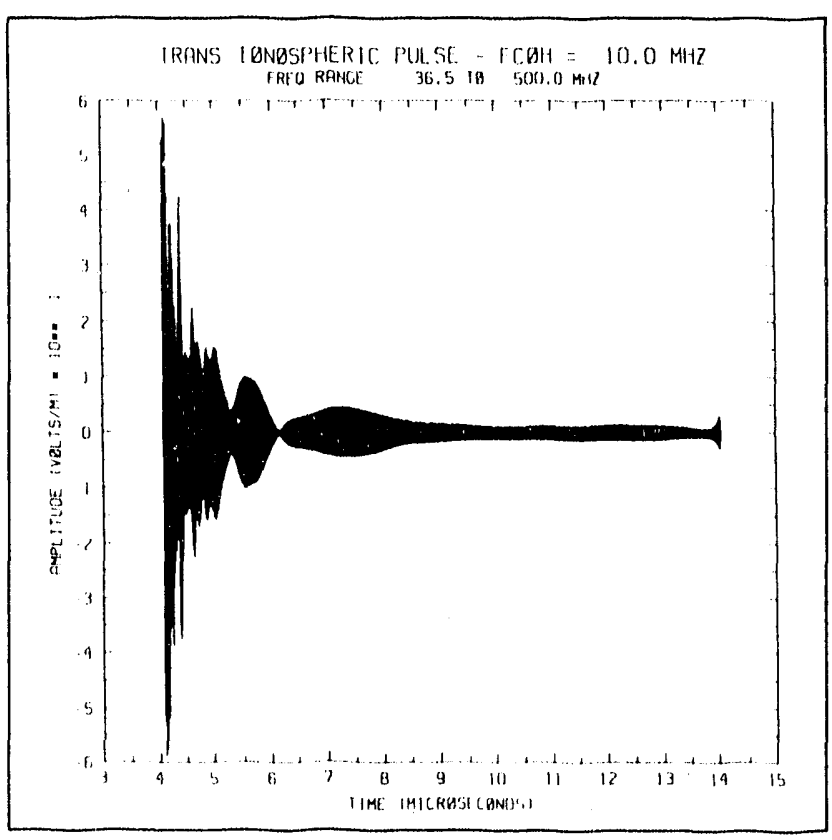

Figure 25c

Figures 25a-c Plots of transionospheric signals obtained for the default delta function, a TEC of $1 \times 10^{120} \mathrm{~cm}^{-2}$ and three sets of parameters (a) infinite coherence bandwidth and 70) $\mathrm{dB}$ SNR, (b) infinite coherence bandwidth and 30 $\mathrm{dB}$ SNR, and (c) 10 MHz coherence bandwidth and $70 \mathrm{~dB}$ SNR. 


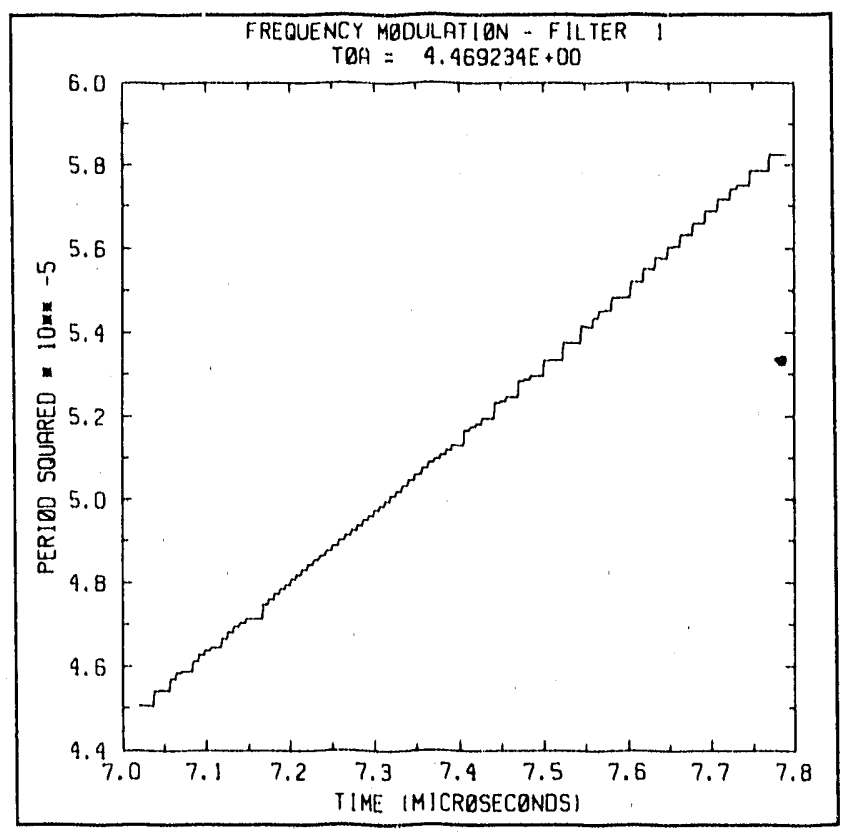

Figure 26a

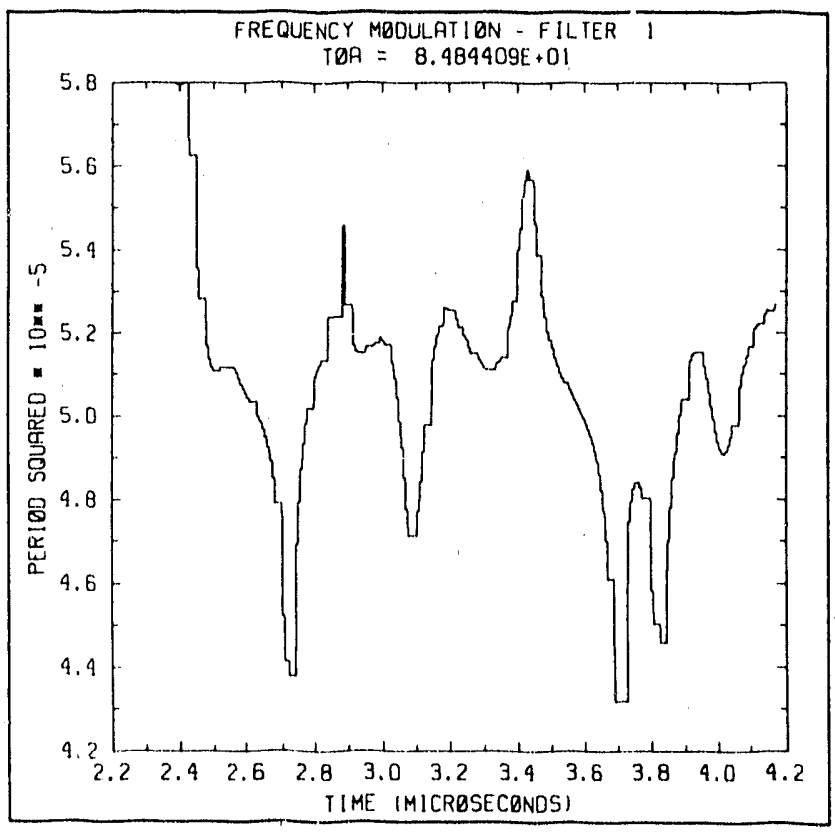

Figure 26b

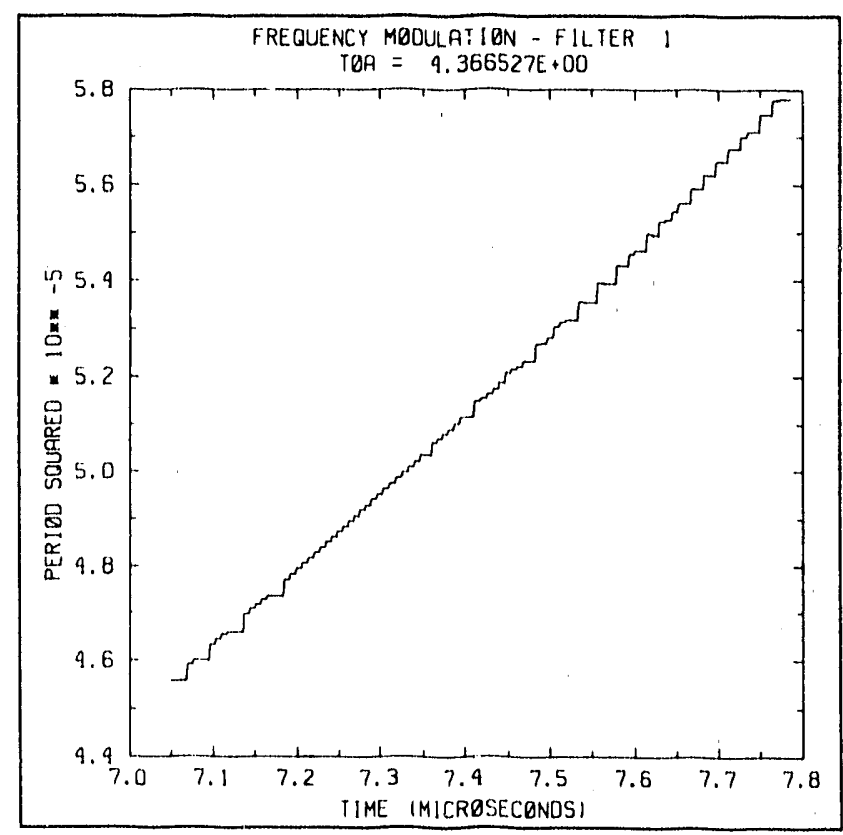

Figure 26c

Figures 26a-c The period squared computed by the FMTOA algorithm plotted vs, time for the wideband filter shown in Figure 24a. The default delta function, a TEC of $1 \times 10^{13} \mathrm{~cm}^{-2}$ and the three sets of parameters (a) infinite coherence bandwidth and $70 \mathrm{~dB}$ SNR, (b) infinite coherence bandwidth and $30 \mathrm{~dB}$ SNR, and (c) $10 \mathrm{MHz}$ coherence bandwidth and 70 dB SNR, are assumed for the three figures, respectively. 


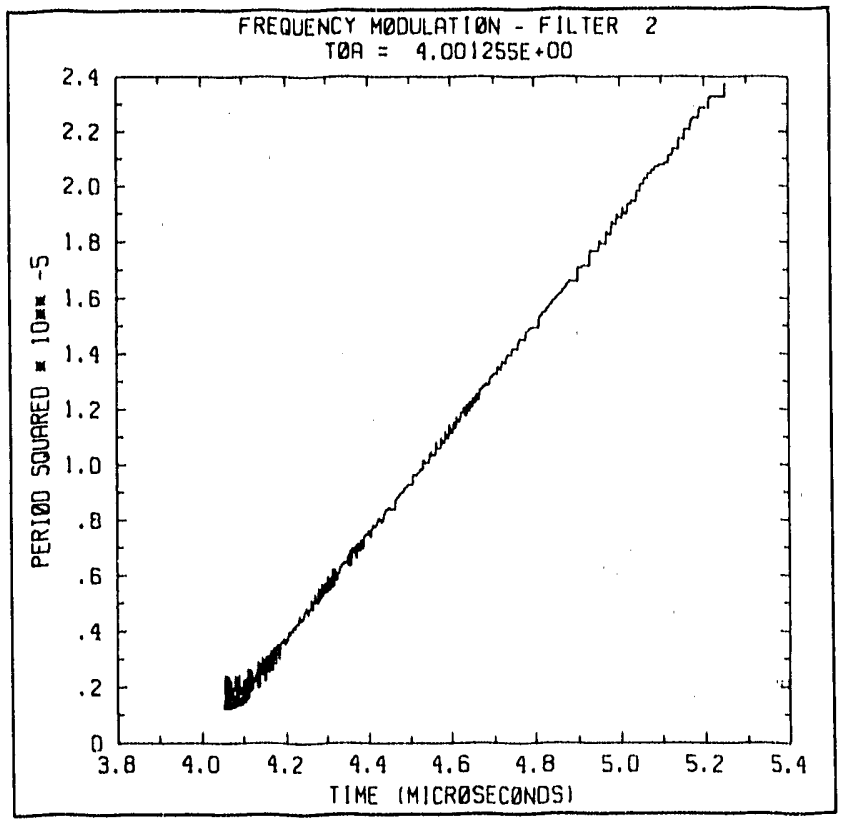

Figure 27a

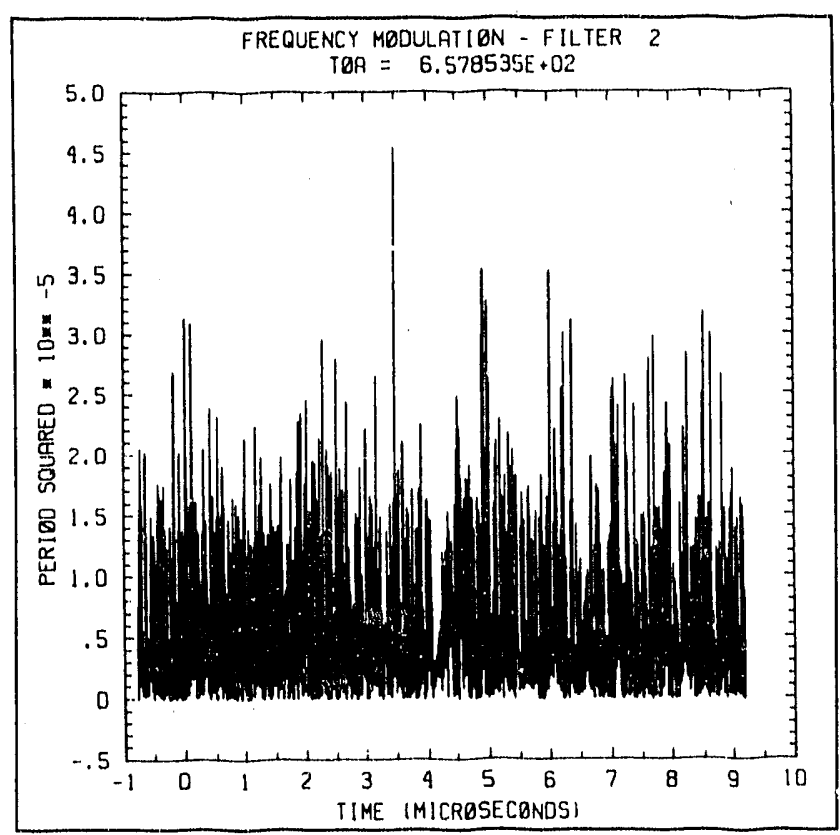

Figure 27b

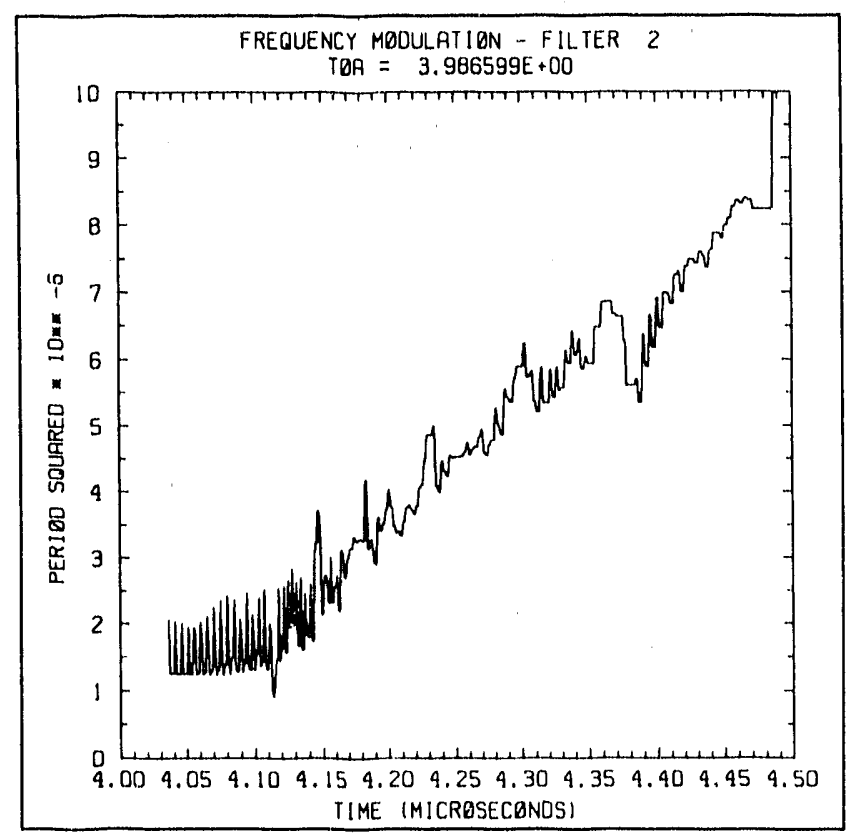

Figure 27c

Figures 27a-c The period squared computed by the FMTOA algorithm plotted $v$ s. time for the narrowband filter shown in Figure 24b. The default delta function, a TEC of $1 \times 10^{13} \mathrm{~cm}^{-2}$ and the three sets of parameters (a) infinite coherence bandwidth and $70 \mathrm{~dB}$ SNR, (b) infinite coherence bandwidth and 30 $\mathrm{dB}$ SNR, and (c) $10 \mathrm{MHz}$ coherence bandwidth and $70 \mathrm{~dB}$ SNR, are assumed for the three figures, respectively. 


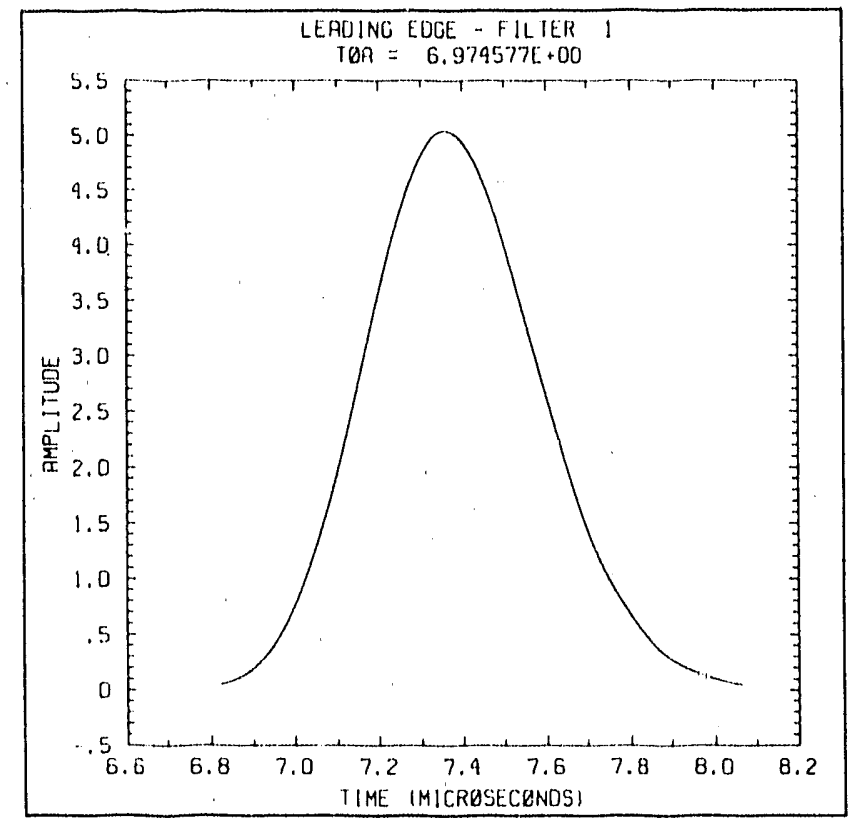

Figure 28a

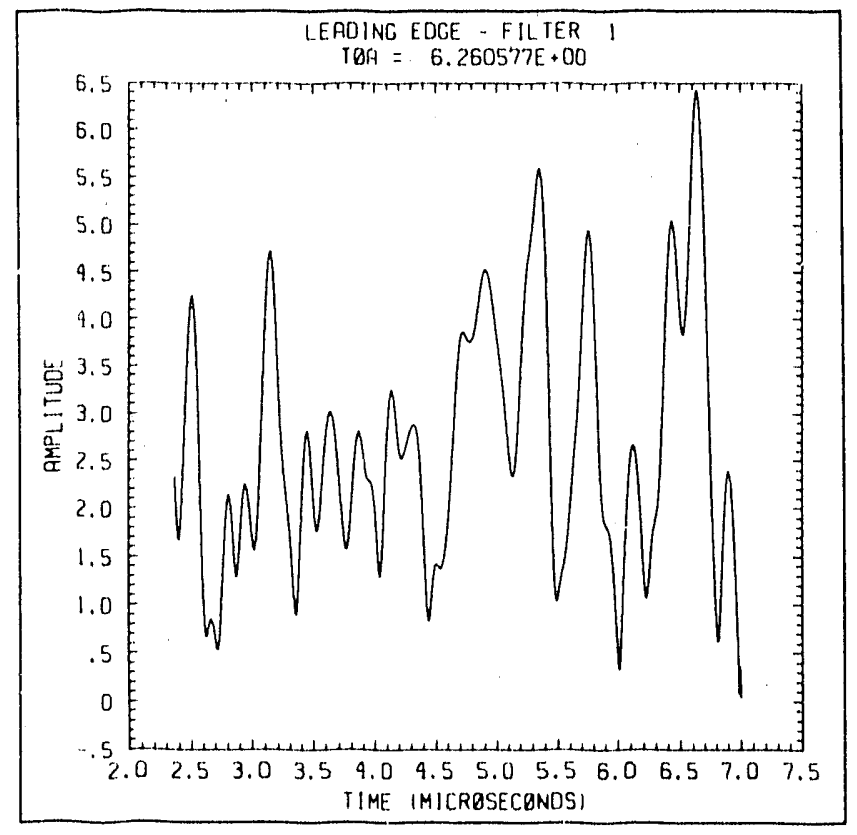

Figure 28b

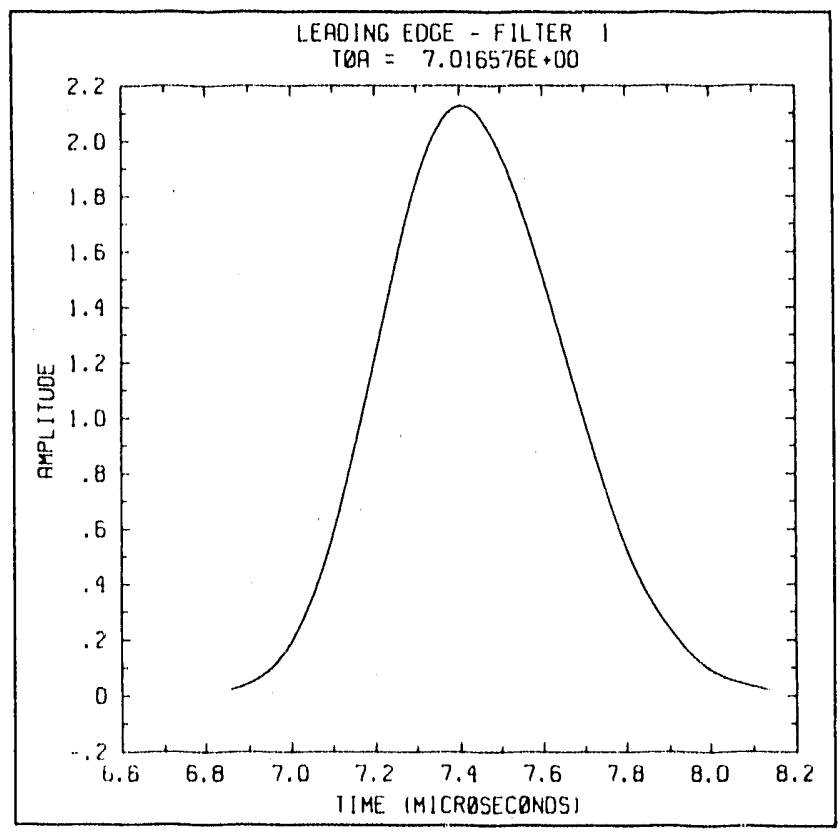

Figure $28 \mathrm{c}$

Figures 28a-c Plot of the envelope of the received signai used by the leading-edge technique to time-tag the arrival of the signal in the wideband filter shown in Figure 24a. The default delta function, a TEC. of $1 \times 10^{1: 3} \mathrm{~cm}^{-2}$ and the three sets of parameters (a) intinite coherence bandwidth and 70) $\mathrm{dB}$ SNR, (b) intinite coherence bandwidth and 30 $\mathrm{dB}$ SNR, and (c) $10 \mathrm{MH} /$ coherence bandwidth and $70 \mathrm{~dB}$ SNR, are assumed for the three figures, respectively. 


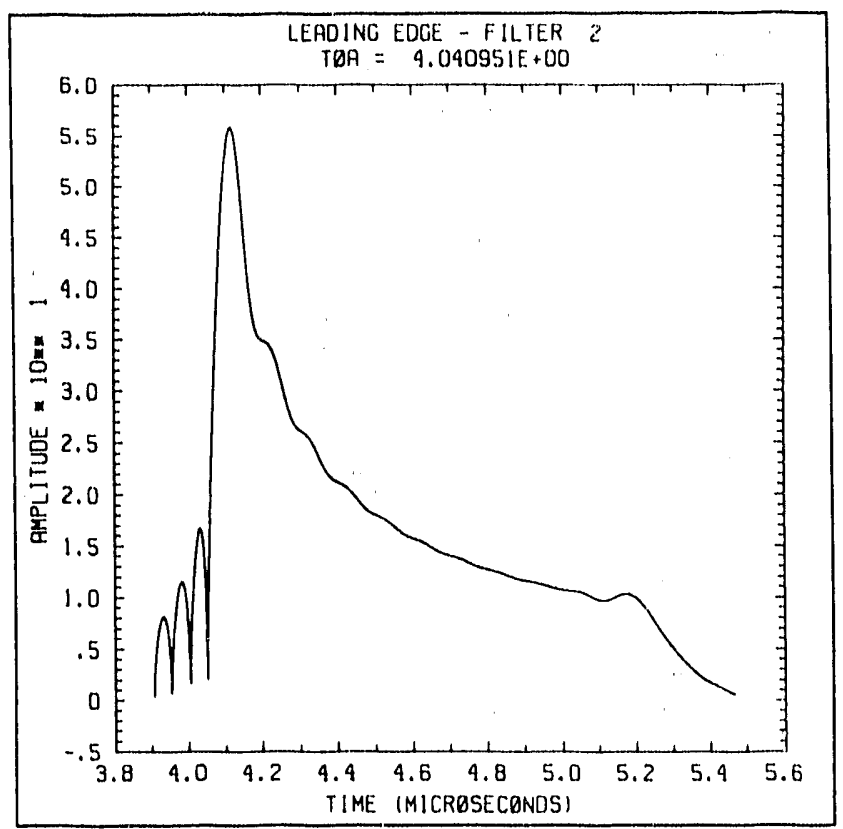

Figure 29a

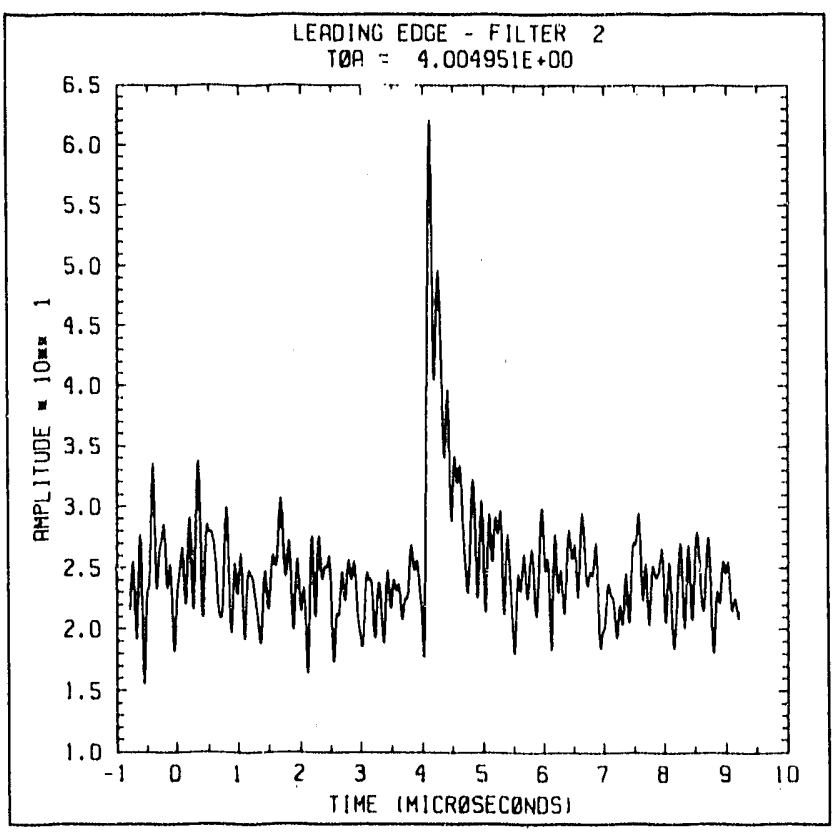

Figure 29b

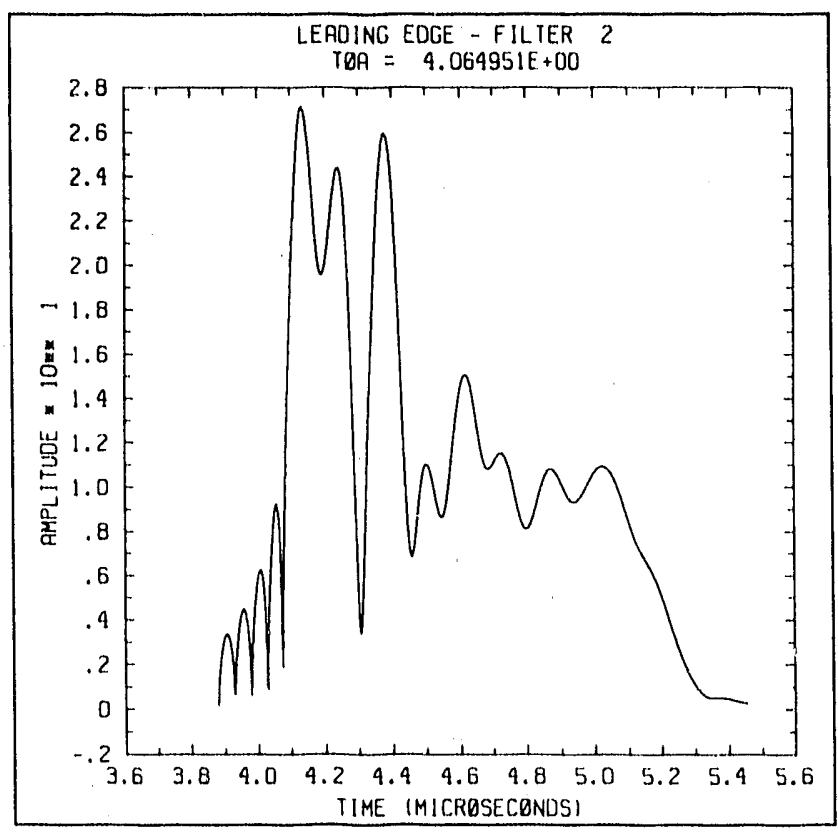

Figure 29c

Figures 29a-c Plot of the envelope of the received signal used by the leading-edge technique to time-tag the arrival of the signal in the narrowband filter shown in Figure 24b. The default delta function, a TEC of $1 \times 10^{13} \mathrm{~cm}^{-2}$ and the three sets of parameters (a) infinite coherence bandyidth and $70 \mathrm{~dB}$ SNR, (b) infinite coherence bandwidth and 30 $\mathrm{dB}$ SNR, and (c) 10 MHz coherence bandwidth and 70) $\mathrm{dB}$ SNR, are assumed for the three figures, respectively. 


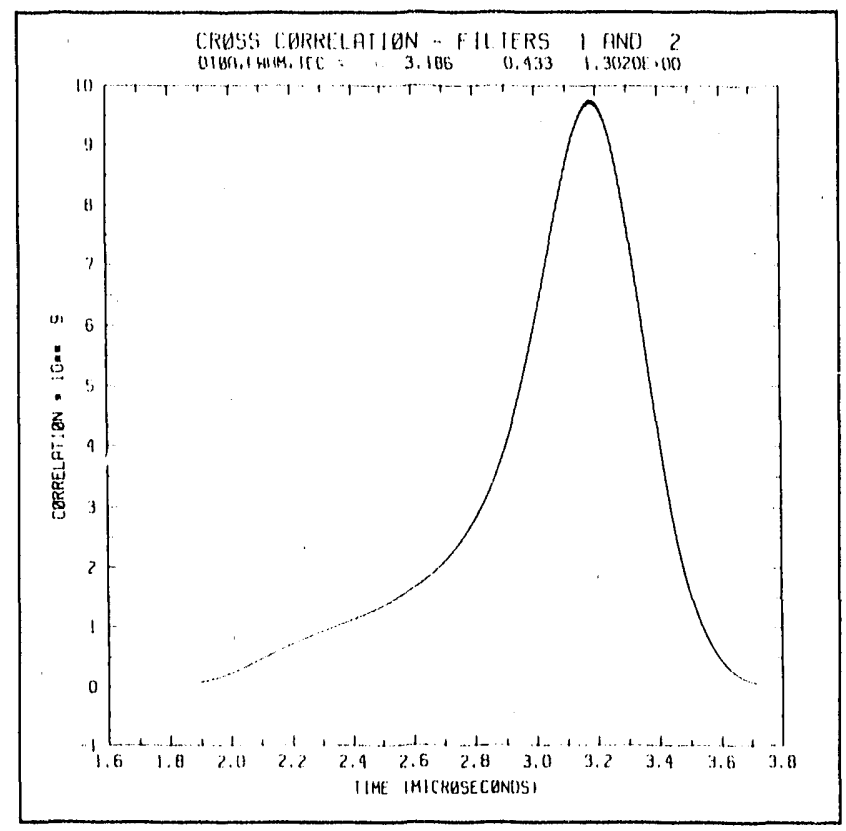

Figure 30a

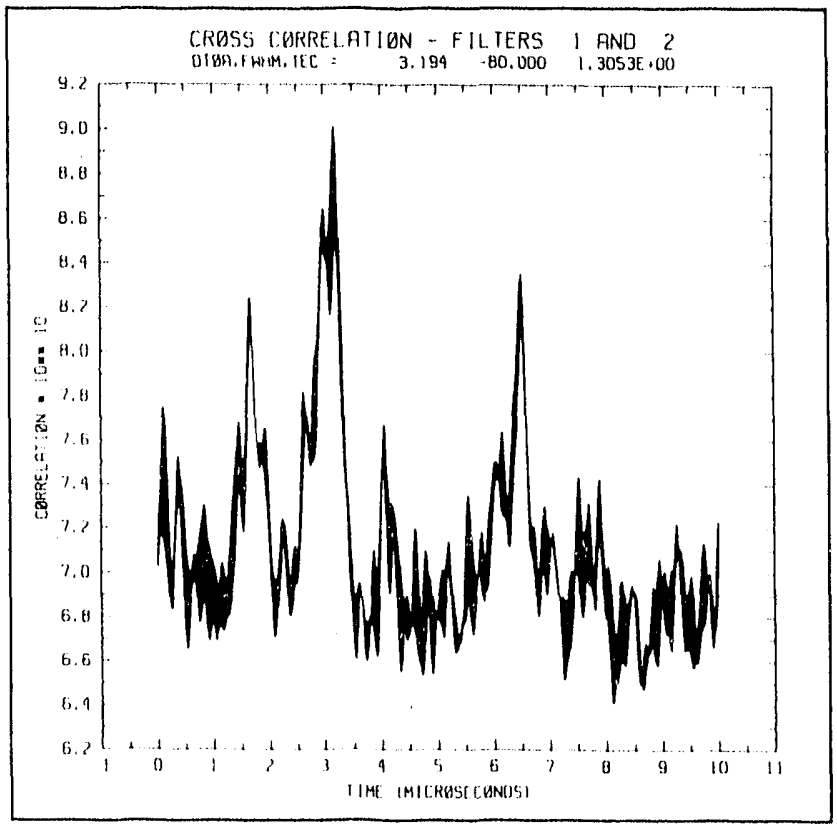

Figure 30b

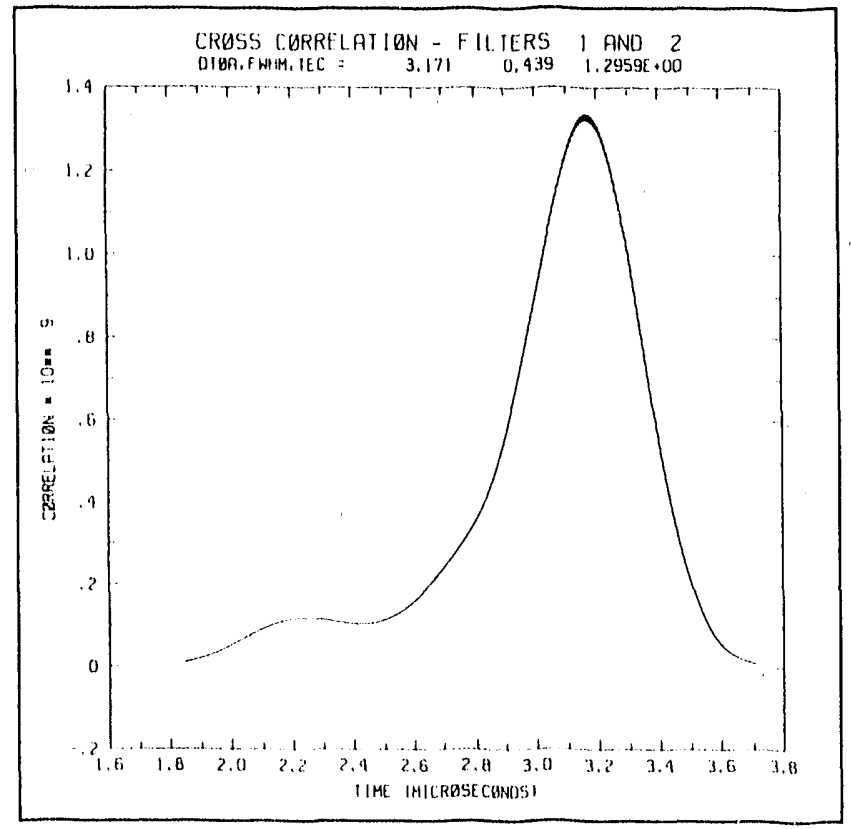

Figure 30c

Figures 30a-c Plots of the cross-correlations of the wideband and narrowband filters shown in Figures 24a and $b$ as a function of the temporal shift $(t)$ between receivers. The default delta function, a TEC of $1 \times 10^{13} \mathrm{~cm}^{-2}$ and the three sets of parameters (a) infinite coherence bandwidth and $70 \mathrm{~dB}$ SNR, (b) infinite coherence bandwidth and $30 \mathrm{~dB}$ SNR, and (c) $10 \mathrm{MH} \%$ coherence bandwidth and $70 \mathrm{~dB}$ SNR, are assumed for the three figures, respectively. 


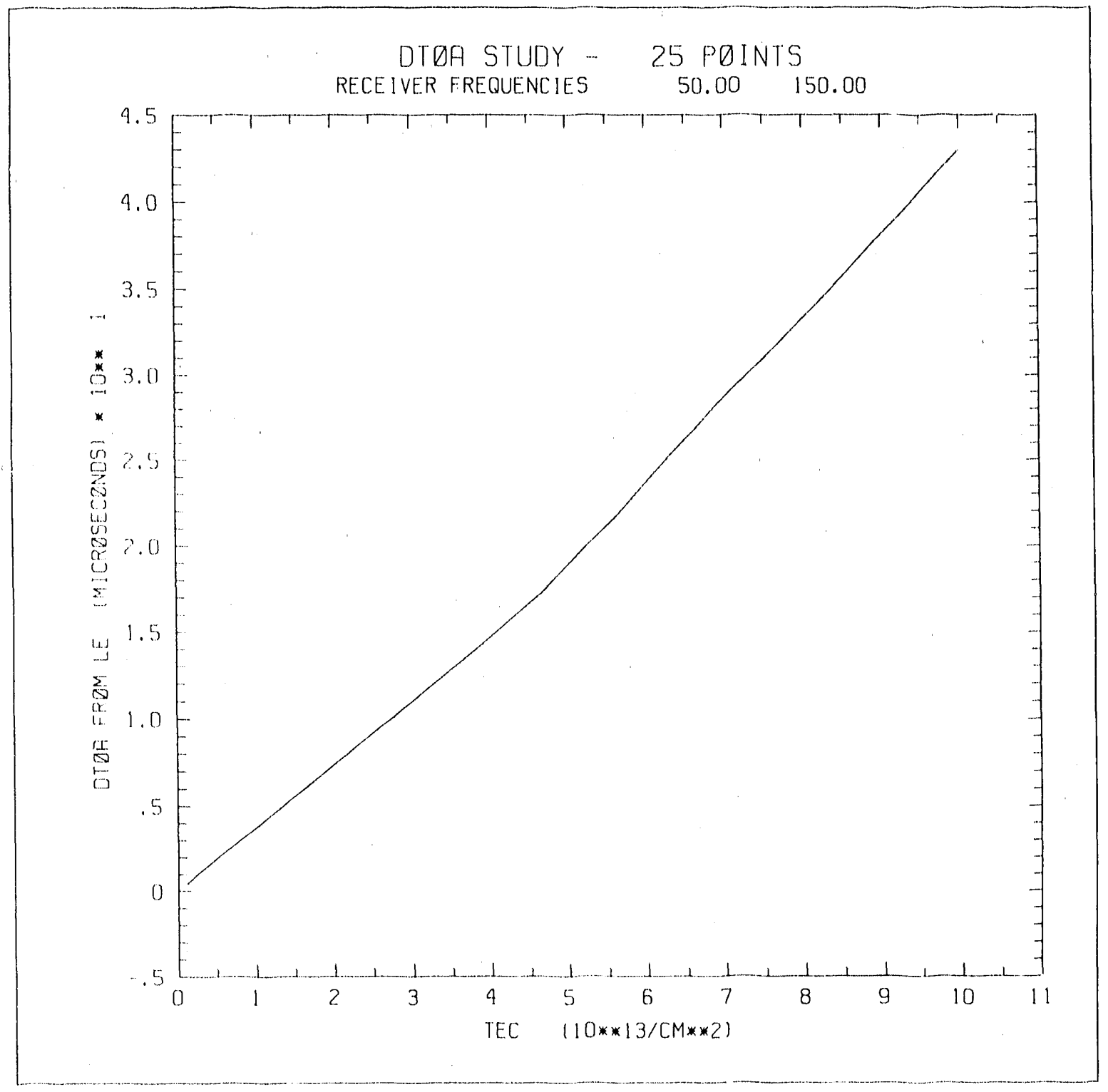

Figure 31 Plot of DTOA, obtained with the leading-edge algorithm for two narrowband receivers with central frequencies of 150 and $50 \mathrm{MHz}$ and a FWHM of $6 \mathrm{MHz}$ vs. TEC for 25 values ranging from $0.1-\ldots$ $10 \times 10^{13} \mathrm{~cm}^{-2}$. The default delta function, $F_{\mathrm{coh}}=\infty$, and $\mathrm{SNR}=70 \mathrm{~dB}$ were used as input parameters. 


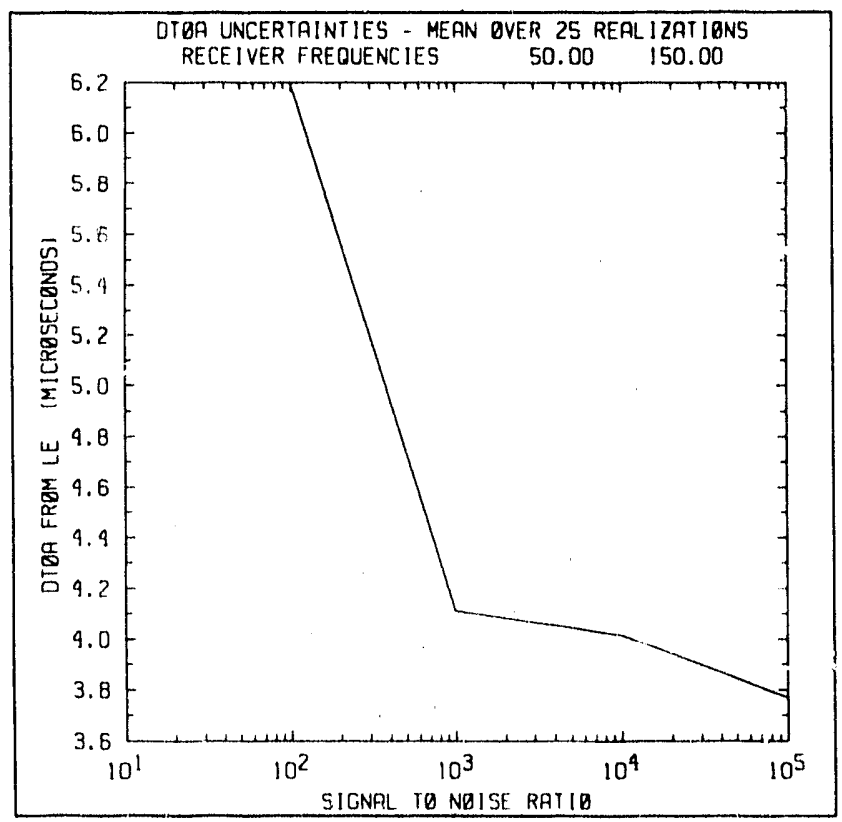

Figure 32a

Figure 32b

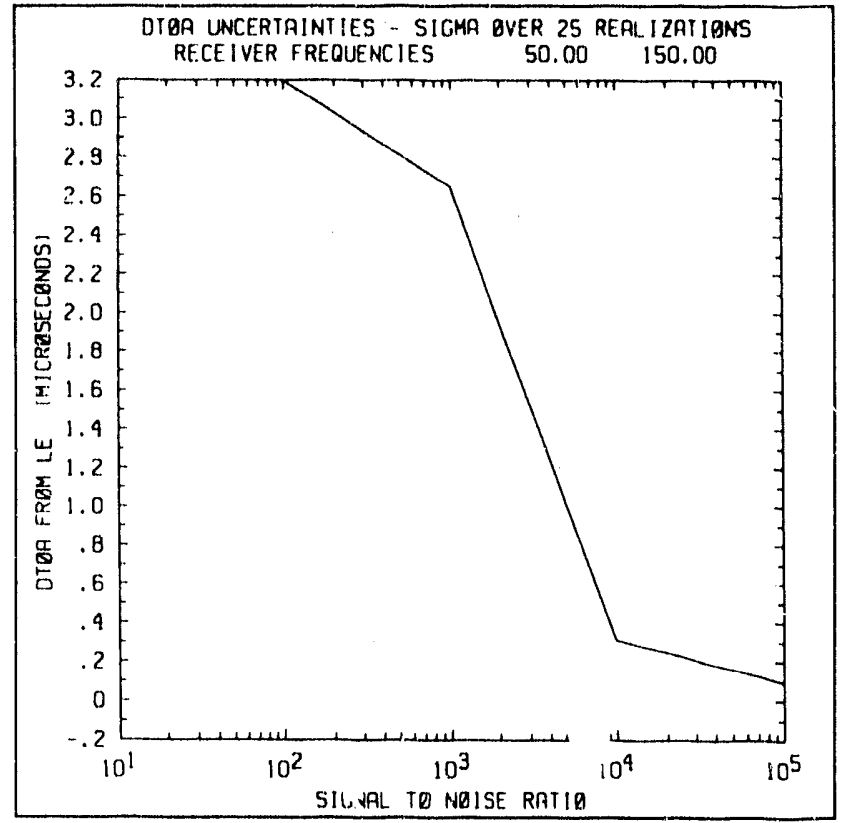

Figure 32a-b Results of the DTOA uncertainty study for two narrowband receivers with central frequencies of 150 and $50 \mathrm{MHz}$ and a FWHM of $6 \mathrm{MHz}$. The TEC was fixed at $1 \times 10^{13} \mathrm{~cm}^{-2}$ and Gaussian white noise was added to the input signal. The resulting filtered transionospheric signal was timetagged with the leading-edge algorithm for SNRs ranging from 102 to 105 . (a) Mean DTOA and (b) standard deviation from the mean were calculated using 25 realizations of each SNR. The default delta function and $F_{\text {coh }}=\infty$ were used as input parameters. 


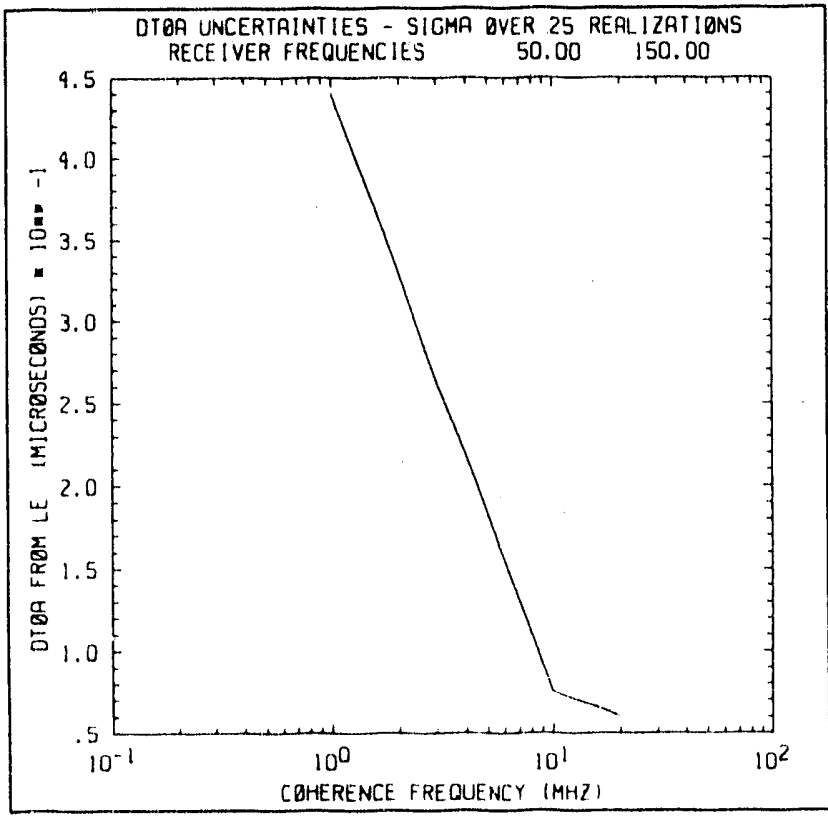

Figure 33a

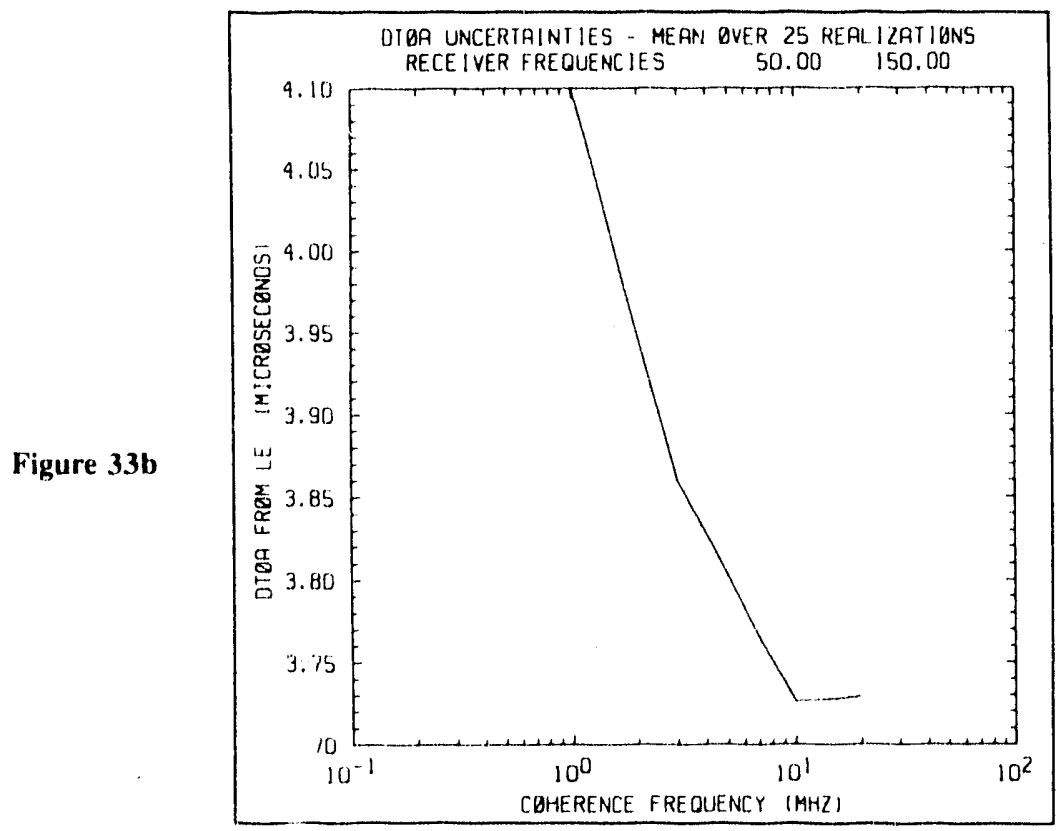

Figure 33a-b Results of the DTCA uncertainty study for two narrowband receivers with central frequencies of 150 and $50 \mathrm{MHz}$ and a FWHM of $6 \mathrm{MHz}$. The TEC was fixed at $1 \times 10^{13} \mathrm{~cm}^{-2}$ and the effects of ionospheric scattering were included. The resulting filtered transionospheric signal was time-tagged with the leading-edge algorithm for $F_{\text {coh }}$ ranging from $11020 \mathrm{MHz}$. (a) Mean D'TOA and (b) standard deviation from the mean were calculated using 25 realizations of each $F_{c o h}$. The default delta function and an SNR of $70 \mathrm{~dB}$ were used as input parameters. 

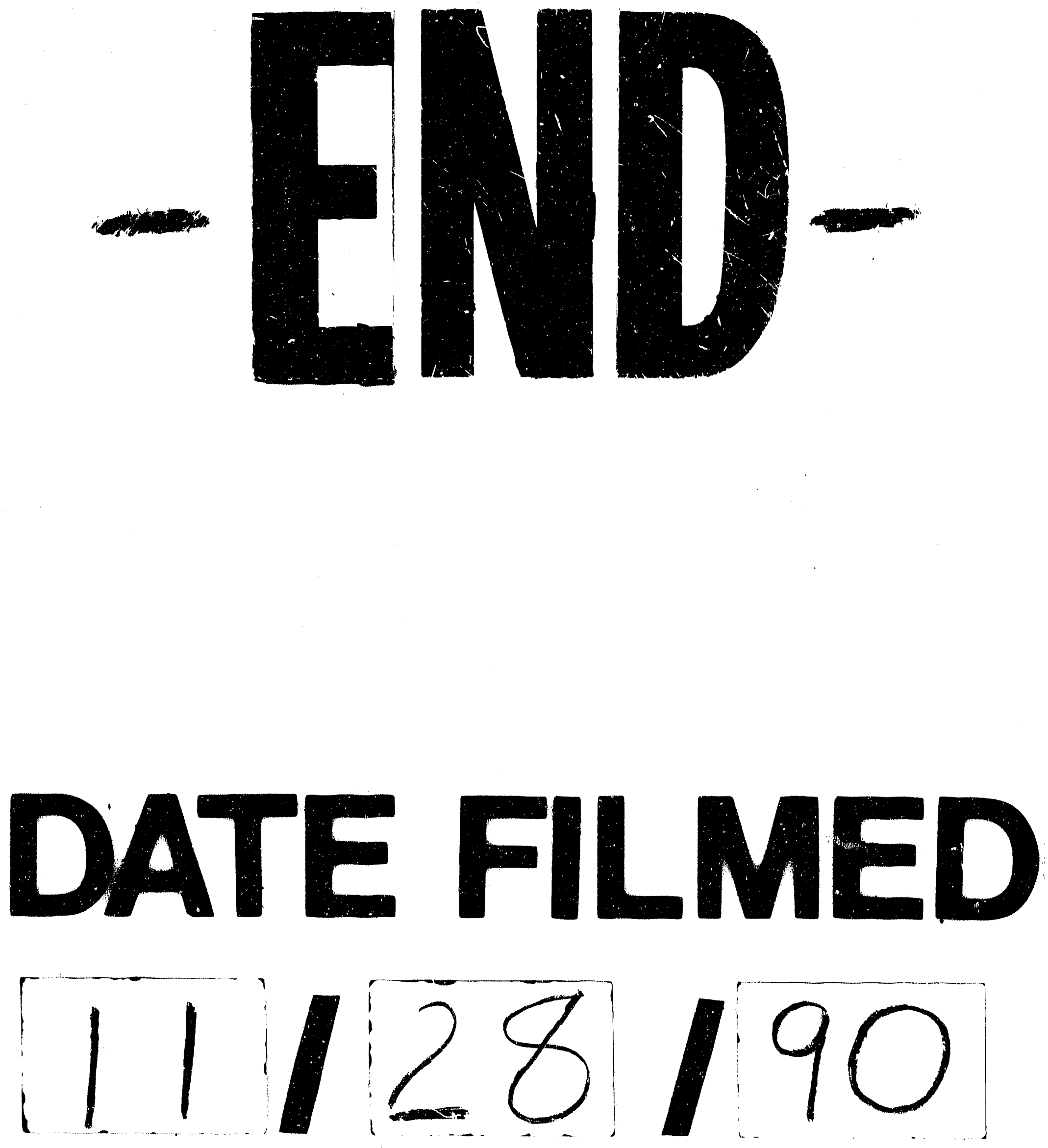
